\title{
A Fingerprint Identification System
}

\author{
By \\ Jean-Christophe Petkovich
}

\begin{abstract}
A thesis proposal submitted to the Faculty of Graduate Studies and Postdoctoral Affairs

in partial fulfilment of

the requirements for the degree of

Master of Computer Science
\end{abstract}

Ottawa-Carleton Institute for Computer Science

School of Computer Science

Carleton University

Ottawa, Ontario

November 2011

(C) Copyright

2011, Jean-Christophe Petkovich 
Library and Archives

Canada

Published Heritage

Branch

395 Wellington Street

Ottawa ON K1A ON4

Canada
Bibliothèque et

Archives Canada

Direction du

Patrimoine de l'édition

395 , rue Wellington

Ottawa ON K1A ON4

Canada
Your file Votre référence

ISBN: 978-0-494-87824-8

Our file Notre référence

ISBN: $978-0-494-87824-8$

\section{NOTICE:}

The author has granted a nonexclusive license allowing Library and Archives Canada to reproduce, publish, archive, preserve, conserve, communicate to the public by telecommunication or on the Internet, loan, distrbute and sell theses worldwide, for commercial or noncommercial purposes, in microform, paper, electronic and/or any other formats.

The author retains copyright ownership and moral rights in this thesis. Neither the thesis nor substantial extracts from it may be printed or otherwise reproduced without the author's permission.
AVIS:

L'auteur a accordé une licence non exclusive permettant à la Bibliothèque et Archives Canada de reproduire, publier, archiver, sauvegarder, conserver, transmettre au public par télécommunication ou par l'Internet, prêter, distribuer et vendre des thèses partout dans le monde, à des fins commerciales ou autres, sur support microforme, papier, électronique et/ou autres formats.

L'auteur conserve la propriété du droit d'auteur et des droits moraux qui protege cette thèse. $\mathrm{Ni}$ la thèse ni des extraits substantiels de celle-ci ne doivent être imprimés ou autrement reproduits sans son autorisation.
In compliance with the Canadian Privacy Act some supporting forms may have been removed from this thesis.

While these forms may be included in the document page count, their removal does not represent any loss of content from the thesis.
Conformément à la loi canadienne sur la protection de la vie privée, quelques formulaires secondaires ont été enlevés de cette thèse.

Bien que ces formulaires aient inclus dans la pagination, il n'y aura aucun contenu manquant. 


\begin{abstract}
Identification and authentication are extremely important tasks as we continue to move into a more information-oriented age. Authentication is becoming necessary for two of the main devices used by nearly everyone today, i.e., laptops/netbooks and cellphones. Identification is becoming an increasingly difficult problem as more and more methods become available to falsify identity.

The use of biometrics is one potential (or partial) solution to both these problems. There are several different possible biometrics that could be of use for either of the above tasks, but few of them have been as widely used, or as closely studied, as fingerprints. This is due to their excellent metrics when it concerns many biometric performance criteria. The permanence, uniqueness and universality of fingerprints make them ideal candidates for use as biometrics.

Fingerprint matching and recognition are extremely difficult problems. Several different important factors are to be considered when attempting to find a fingerprint match, and of them, the most important are distortions. These potential distortions significantly increase the complexity of matching or recognition. A noisy fingerprint can contain a number of different types of distortion including rotation, displacement, distortion due to skin plasticity, variance in pressure when the print is taken, condition of the skin when the print is taken, and the noise caused by the equipment (for example, as a result of the use of older fingerprint collection techniques).

Fingerprint matching, along with other biometric matching techniques, follows a relatively well-established process streamlined as follows: collection, pre-processing, extraction, and finally matching. The focus of this thesis is the comparison of different techniques centered around the matching of minutiae. In this research endeavor, we have studied all the associated processes with achieving this. Besides, we have also developed a fully-functioning prototype system to collect fingerprints, pre-process them, extract minutiae, and to finally compare and combine several different matching techniques. Finally, we will be able to accomplish a comparison of existing techniques
\end{abstract}


based on the minutia-matching paradigm that also incorporates noisy subsequence tree comparisons. 


\section{Acknowledgments}

Firstly, I would like to thank Prof. John Oommen for accepting me into his lab, for his support and expertise. His experience and methodical approach were invaluable in the development of this thesis.

I would also like to thank my family for all their love and support, without which I would surely not have been able to complete this thesis. In particular, I would like to thank my mother and father for their encouragement and valuable guidance. I would also like to thank my sister for her support and her assistance during this period.

Finally, I would like to thank Carleton's School of Computer Science. I have met some brilliant and exceptional people, and I have also learned much since my arrival here two years ago. The people I have met here have shaped both my interests and ambitions, and will likely have a great impact on the rest of my life. 


\section{Contents}

1 Introduction 1

1.1 Motivations and Objectives ................. 1

1.2 Problem Statement . . . . . . . . . . . . . . . 2

1.3 Contributions ....................... 4

1.4 Scope .......................... 7

1.5 Outline .......................... 7

2 Background: Survey of the Field 9

2.1 What is a Biometric System? . . . . . . . . . . . . . 9

2.2 Significant Properties and Trade-offs of Biometric Systems . . . . . . 11

2.2.1 Verification vs. Identification . . . . . . . . . . 12

2.2 .2 Automated vs. Attended . . . . . . . . . . . . . 13

2.2.3 Trade-off: Matching Speed vs Accuracy . . . . . . . . . . . . . 13

2.2.4 Trade-off: Biometric Types . . . . . . . . . . . . . . 14

2.3 Why Fingerprints are Effective Biometrics . . . . . . . . . . 16

2.4 Fingerprint Distinctiveness and Validity/Accuracy as a Biometric . . 17

2.4.1 Admissibility in Court . . . . . . . . . . . . . 19

2.5 Biology of Fingerprint Uniqueness . . . . . . . . . . . . . . . . 19

2.6 The Fingerprint Identification Process . . . . . . . . . . . 20

2.6.1 Raw Data Collection ... . . . . . . . . . . 20

2.6.2 Data Enhancement ............... 24

2.6.3 Feature Extraction and Template Construction . . . . . . 25

2.6.4 Minutia Matching . . . . . . . . . . . . . 30 
2.7 Non-Minutia-based Matching Techniques . . . . . . . . . . . . 33

2.7 .1 Refining Results . . . . . . . . . . . . . . . 34

2.8 Chapter Summary . . . . . . . . . . . . . . . . . . . . . . 34

3 Image Processing Aspects $\quad 36$

3.1 Adding Artificial Noise . . . . . . . . . . . . . . . 37

3.1.1 Convolution with Arbitrary Kernels . . . . . . . . . . . . 39

3.1 .2 Pixel-Damage-Based Noise . . . . . . . . . . . . . . . . . . 39

3.1 .3 Additive Gaussian Noise . . . . . . . . . . . . . . . . . 40

3.2 Image Enhancement Methods . . . . . . . . . . . . . . . . 42

3.2 .1 Gaussian Blur . . . . . . . . . . . . . . . . . . 42

3.2.2 Contrast Adjustment and Normalization . . . . . . . . 42

3.2.3 Gabor-Filtering-Based Fingerprint Image Enhancement . . . . 43

3.3 Additional Image Processing Tools Used . . . . . . . . . . . . . 44

3.4 Chapter Summary . . . . . . . . . . . . . . . . . . . . 45

4 File Format Considerations $\quad 46$

4.1 Importance of Compression $\ldots \ldots \ldots \ldots$

4.2 Problems with Pre-Existing Image Compression Techniques . . . . . 47

4.3 Wavelet Scalar Quantization Compression . . . . . . . . . . 47

4.4 File Formats Used in The Developed System . . . . . . . . . . . 50

4.5 Chapter Summary . . . . . . . . . . . . . . . . 51

5 Pattern Recognition Methods $\quad 52$

5.1 Survey of Global Matching Techniques _. . . . . . . . . . . 52

5.1 .1 Hough Transform . . . . . . . . . . . . . . 53

5.1 .2 Ridge Feature Alignment Approach . . . . . . . . . . . 53

5.2 Survey of Local Matching Techniques . . . . . . . . . . . . . . 54

5.2 .1 Feature-Vector Based Matching . . . . . . . . . . . . 54

5.2 .2 Adjacency Graph Matching . . . . . . . . . . . . 55

5.2.3 Geometric Clustering-Based Matching . . . . . . . . . 55

5.3 Implemented Recognition Methods . . . . . . . . . . . . . 56 
5.3 .1 Development Notes . . . . . . . . . . . . . . . 56

5.3.2 Global-Scheme: Hough Transform Alignment-based Matching 57

5.3.3 Local-Scheme: K-plet Representation Matching . . . . . . . 64

5.3.4 Local-Scheme: Bozorth's Algorithm . . . . . . . . . . . . . 68

5.4 Chapter Summary . . . . . . . . . . . . . . . 70

6 Subsequence-Tree-Based Methods $\quad 71$

6.1 Noisy Subsequence Tree Recognition Problem . . . . . . . . . . 71

6.1.1 NSTRP and Fingerprint Matching Problem Similarities . . . . 72

6.2 Adapting Noisy Subsequence Tree Recognition . . . . . . . . 76

6.2.1 Encoding Minutiae and Their Local-Spatial-Relationships . . . 77

6.2 .2 Noise Considerations . . . . . . . . . . . . . . . 80

6.3 Subsequence Algorithm Performance . . . . . . . . . . . . 81

6.4 Using Artificial Noise . . . . . . . . . . . . . . . . . . . . 82

6.4.1 Results Analysis/Future Work . . . . . . . . . . . 83

6.5 Chapter Summary . . . . . . . . . . . . . . 85

7 Overall System $\quad \mathbf{8 6}$

7.1 System Features . . . . . . . . . . . . . . . . . 88

7.2 Design Process/Rationale . . . . . . . . . . . . . 88

$7.2 .1 \quad$ Fingerprint Library . . . . . . . . . . . . . . 90

7.2 .2 The Piping Mechanism . . . . . . . . . . . . . . 91

7.2 .3 Combined Components . . . . . . . . . . . . . . . 92

7.3 System Components . . . . . . . . . . . . . . . . . . 93

7.3.1 Raw Data Collection Phase . . . . . . . . . . . . 94

7.3.2 Data Enhancement Phase . . . . . . . . . . . . . 94

7.3.3 Feature Extraction Phase and Template Construction . . . . 9 95

7.3.4 Minutia Matching Phase . . . . . . . . . . . . . . 96

7.4 Usage Examples . . . . . . . . . . . . . . . . . . . . . . 96

7.5 Chapter Summary . . . . . . . . . . . . . . . . . . 99 
8 Conclusions $\quad 100$

8.1 Thesis Summary . . . . . . . . . . . . . . . 100

8.2 Summary of Contributions . . . . . . . . . . . . . . . 101

8.3 Summary of Conclusions . . . . . . . . . . . . . . . . 103

8.4 Future Work . . . . . . . . . . . . . . . . . . . 104

$\begin{array}{ll}\text { Bibliography } & 105\end{array}$ 


\section{List of Figures}

2.1 General structure of a fingerprint scanner. Drawn from a description reported in $[1] . \ldots \ldots \ldots \ldots \ldots \ldots \ldots$

2.2 Illustration of an orientation window on a group of ridges with similar $\theta$ values. Diagram similar to that in $[2] \ldots \ldots \ldots \ldots \ldots$

2.3 The different types of minutiae. From left to right, are the ridge bifurcation minutia, the ridge-ending minutia, and the short ridge minutia.

2.4 The minutiae on this fingerprint have been highlighted with red squares, the blue line indicates the direction of the most closely associated ridge, this is known as the minutia's $\theta$ value. . . . . . . . . . . .

3.1 Examples of the different types of noise which can be applied. From left to right and top to bottom, we have the original image, the image with Gaussian additive noise applied, the image with pixel damage (salt and pepper) noise applied, and finally with Gaussian blur (convolution with a Gaussian Kernel) applied. . . . . . . . . . . . . . . . .

4.1 Examples showing the effects of two different image compression types. In the left panel is a fingerprint image compressed using standard JPEG compression and in the right panel the same image compressed using the FBI's standard WSQ compression. The compression algorithms were tuned to the same compression ratio. The magnified portion of the left image shows the blocks produced by the JPEG compression algorithm. (Note: The effect may not be as pronounced in some PDFreaders due to image processing performed by these applications.) . . 
5.1 Diagram showing the alignment produced by Algorithm 5. The top two images depict the two fingerprints, from the same digit, with their detected minutiae, and the bottom image is the resulting alignment between the two sets of minutiae. One set of the minutiae is coloured green with its $\theta$ indicator coloured orange, while the other set of minutiae is coloured red with its $\theta$ indicator coloured blue. . . . . . . . . .

6.1 Diagram illustrating the concentric circles which define the levels of the minutia tree. The minutiae are highlighted with red squares, the angle of the most closely associated ridge is indicated with a blue line, and the child-parent relationships are shown in green. . . . . . . .

6.2 Diagram illustrating what occurs when one of the bands formed by the concentric circles is empty. The empty band is highlighted in pink, and is joined with the next smallest concentric circle indicated with a dotted line. These two now form the first level (root) of the tree. . . .

7.1 Diagram illustrating the overall system, shows each stage and the filetype/state of the information as it moves through the system. . . .

7.2 Diagram of the fingerprint class, showing the main externally-visible fields of the class. . . . . . . . . . . . . . . . .

7.3 Usage examples of basic interactions with the "afis" command-line utility including: matching a file against a database, registering a file and then matching it against the database and garbling the image prior to

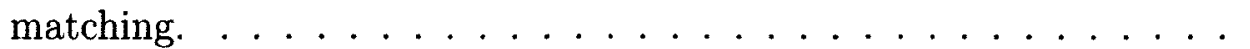

7.4 Usage examples of several more advanced options of the "afis" utility including: selection of the matching algorithm(s), specifying noise options, specifying options to the garbling algorithms. . . . . . . .

7.5 Usage examples of supplying different algorithms for pre-processing and feature extraction. . . . . . . . . . . . . . . . . 


\section{Chapter 1}

\section{Introduction}

\subsection{Motivations and Objectives}

Fingerprint matching analysis has been a useful measure of identity and authenticity for over 2,000 years, first in China as a method of authenticating documents [3]. Fingerprint identification and verification is based on the assumption that no two individuals share identical fingerprint patterns [3]. Fingerprint ridge and pore patterns (in whole or in part) can be transferred to solid surfaces by deposition of contact residue comprised of skin cells, oil, salt and moisture, or optically captured to provide a two-dimensional representation [3]. Such representations can be stored indefinitely in photographic or digital form, facilitating comparison against fingerprint archives. The process of determining a fingerprint match entails the evaluation of spatially distributed components of fingerprints that are in alignment as opposed to those that are not.

The processes involved in the gathering and storage of prints generate distortions, artifacts and noise which need to be managed so as to increase the accuracy and precision of any fingerprint matching system [4]. Very often, only partial representations of the prints are available, further increasing the complexity of the pattern matching process. Although fingerprint recognition is a well-studied problem, matching highly fragmented fingerprints is a very difficult problem, and is widely considered to be unsolved [1]. 
The objectives of this thesis encompassed three main tasks. First, we decided to develop a detailed study of biometric systems, including the design, specification and construction of a complete fingerprint recognition system. Such a system could be viewed as a "pipeline" of data which has a number of components characterizing its functionality. We had to understand, then collect or design and implement all the necessary components, or modules, of the final system. The modules involved included raw data collection, image processing and enhancement, feature extraction and template construction, and finally minutia matching. In addition to the components and functionality typically included in a fingerprint recognition system, we wished to create a reusable and flexible fingerprint recognition system which could be extended to the user's/developer's liking. The construction of a fingerprint recognition system, as well as the task of studying and implementing several different algorithms available in the literature, provided us with insight into the issues related to our next task the implementation of a new approach to fingerprint matching.

We wished to adapt the noisy subsequence recognition algorithm reported in [5] to the fingerprint recognition problem. Previously, the noisy subsequence recognition algorithm performed exceptionally well on a synthetic problem which bore a striking resemblance to the fingerprint recognition problem. This motivated us to attempt to adapt the algorithm to the fingerprint matching problem.

Third, and finally, we needed to evaluate the performance of our novel pioneering algorithm against that of other well known fingerprint matching algorithms.

\subsection{Problem Statement}

Before we proceed, it is useful to precisely define the requirements and the components of a fingerprint recognition system. These components represent different phases of the fingerprint matching process, each of which play an important role in the accurate matching of fingerprints. These components include the following:

1. Raw Data Collection: Given a physical finger, the component should be able to produce either an image of the rolled or pressed impression of the finger, referred to as a fingerprint image, $I$. $I$ is often a simple array of pixel intensities. 
2. Fingerprint Image Enhancement: From $I$, this component should be able to produce an enhanced fingerprint image, $E$. $E$ may either be masked with a quality map or could have been enhanced for the purposes of fingerprint matching. Enhancements which typically occur are either contrast adjustment and ridge thinning, or ridge recovery through Gabor filtering.

3. Feature Extraction: Feature extraction involves the conversion of the enhanced fingerprint image, $E$, into quantitative data regarding its ridge features. This data is usually associated with specific locations referred to as minutiae. The term "minutia" is used to refer to small ridge details found in fingerprints; most often, they are a set of characterizing points described on ridge structures ${ }^{1}$. The component should output a set of minutiae or similar features, $M$, which is also commonly referred to as a template ${ }^{2}$.

4. Feature Matching ${ }^{3}$ : The matching phase concerns itself with the fingerprint matching problem, and is described in detail below.

The fingerprint matching problem itself is slightly more complex than the problems tackled in the other phases listed above. As an understanding of the fingerprint matching problem will be critical to the appreciation of the central problem of identification/authentication through fingerprints, a definition of the problem is provided below.

Given a database of fingerprint minutiae sets $\mathcal{D}=\left\{M_{1}, M_{2}, \ldots, M_{d}\right\}$, determine which fingerprints, if any, are from the same digit as a probe fingerprint minutiae set $\mathcal{P}$. Associated with this central question are a number of different sub-problems, or approaches, which are more specific, the most common of which is the "fingerprint minutia matching problem".

The fingerprint minutia-matching problem is given greater focus in this thesis as it has become the "de facto standard" for fingerprint matching [4].

\footnotetext{
${ }^{1}$ The most common features and their associated metrics are detailed in Chapter 2 (Section 2.6.3).

${ }^{2}$ Fingerprint templates may also hold meta-data such as the known source of the fingerprint, but this meta-data is not strictly necessary.

${ }^{3}$ Also referred to as the minutia-matching phase.
} 
The minutia-matching problem can be defined as follows: Let $\mathcal{P}$ and $\mathcal{Q}$ be two sets of minutiae each representing separate fingerprints:

$$
\begin{aligned}
& \mathcal{P}=\left\{p_{1}, p_{2}, \ldots, p_{m}\right\}, \text { where each } p_{i}=\left\{a_{1}, a_{2}, \ldots, a_{k}\right\} \\
& \mathcal{Q}=\left\{q_{1}, q_{2}, \ldots, q_{n}\right\}, \text { where each } q_{j}=\left\{b_{1}, b_{2}, \ldots, b_{k}\right\}
\end{aligned}
$$

where $m$ is the number of minutiae discovered in the fingerprint associated with $\mathcal{P}$, $n$ is the number of minutiae discovered in the fingerprint associated with $\mathcal{Q}, a$ and $b$ are minutia attributes, and $k$ is the number of different minutia attributes.

Minutiae $p_{i}$ and $q_{i}$ are considered to match if their attributes are within a certain threshold ${ }^{4}$. Further, two fingerprints are reckoned to be "matching" if their computed "matching score", which is most often a function of the number of matched minutiae between them, attains or exceeds a defined threshold.

While this is a basic formulation of the problem, one should understand that as there is significant noise involved in fingerprint matching, there are many different methods for computing whether two minutiae match, and further, if two fingerprints themselves match.

For the purposes of this thesis, we will refer to the fingerprint matching problem as the "general fingerprint matching problem" and to the more restricted fingerprint minutiae matching problem as the "fingerprint matching problem" ${ }^{5}$.

\subsection{Contributions}

The research process reported in this thesis has led to a number of contributions. The first is the design and implementation of a comprehensive, flexible, high-level fingerprint recognition library. The library is composed of several different tools

\footnotetext{
${ }^{4}$ As we shall see later, this is an oversimplified model as there are a number of methods that do not conform to this problem specification, and so perform various transformations on the minutiae sets in order to match them together. However, this model remains useful as an illustration of the central fingerprint matching problem.

${ }^{5}$ There are a number of different physical features used to match fingerprints, but matching based on minutiae is, as mentioned, the de facto standard.
} 
and modules. Where possible, the use of already-existing open source software tools and libraries for use with fingerprint recognition were used, avoiding duplication of previous efforts. The library is easily scriptable, providing a high-level interface to the fingerprint recognition process, and is extensible through both calls to the system shell and also the several dynamic library interfaces available in Python (e.g., ctypes). All the details involving the conversion between image formats, the decoding of WSQ files, and extracting minutia data are hidden from the user.

The processing that is hidden from the user by our library encompasses three out of the four main components of a fingerprint matching system, namely, the collection of the raw data, fingerprint image enhancement and feature extraction.

The raw data collection is handled by the library through the use of the open source fingerprint scanner library "fprint". Although supported by our library, in order to avoid the manual collection of large enough quantities of fingerprints for testing, we procured several databases of fingerprints from the FVC2000 and FVC2002 fingerprint competitions. In addition to the collection of raw fingerprint images, it is essential to be able to convert between different image formats in order to take advantage of open source software ${ }^{6}$. Most of the available open source tools for fingerprint recognition use either the WSQ, JPEG, TIFF, or PNG image files as their input or output. We have used the "cwsq", "dwsq", and "PIL" tools/modules to enable the conversion of our database images into the other formats. The details of all these issues are described in Chapter 4 of the thesis.

The enhancement provided by our library includes contrast normalization and a heuristic to determine the quality of disparate regions of the fingerprints. This functionality was added through the use of the "mindtct" program.

Feature extraction is pivotal to the function and performance of a fingerprint recognition system. The fingerprint ridge-features used by our library are primarily the minutiae. Extraction is performed through the use of the "mindtct" program. In our library, we support two different minutia file-types: XYT and MIN files. The XYT files contain less information but are smaller, and consequently, they are simpler to

\footnotetext{
${ }^{6}$ As tools are often designed for a specific input format, the flexibility to convert between as many formats as possible permits choosing from a larger set of potential tools.
} 
parse whereas MIN files store many diverse pieces of data about each minutia. These details are again explained in Chapter 4.

The library also offers parallelism by executing individual matching processes between two fingerprints in parallel. It is not necessary for the user to enable or configure this feature as it is applicable to all fingerprint matching techniques.

In addition to the fingerprint library, we have created a complete fingerprint recognition system. As with the fingerprint library from which it was constructed, our fingerprint recognition system is scriptable, extensible and capable of parallelism.

This fingerprint recognition system which we have developed is capable of constructing arbitrary hierarchical fingerprint matching systems by permitting the "piping" of results from one algorithm to another. This allows a user to take advantage of different pre-processing techniques and the desirable properties from several different fingerprint matching algorithms and/or compensating for the weaknesses in different algorithms.

Our third contribution is the implementation of a novel and pioneering fingerprint recognition algorithm based on a solution to the noisy subsequence tree recognition problem (NSTRP) reported in [5]. This algorithm adapts the noisy subsequence tree recognition algorithm (NSTRA) to compare fingerprint minutiae information encoded into noise tolerant trees.

As part of our adaptation of the NSTRA, we have developed a method of encoding fingerprint minutiae information into noise-tolerant trees. In addition to its use in the NSTRA-based algorithm, we believe that it could prove effective when combined with other matching techniques. A method similar (in concept) to this was earlier reported in [6], which was intended for use with specialized graphs, and we believe that this scheme could be adapted for use with the trees produced by our method. The specific features of the noise-tolerant tree representations produced by our technique render them to be useful for fingerprint recognition because of their elimination of rotation distortion, and their dampening of the effects of displacement distortion associated with matching fingerprints arising from different sources. 


\subsection{Scope}

In this thesis we have described a broad perspective of the field of fingerprint recognition. To achieve this, we have covered biometric systems in detail, the effectiveness of fingerprints as biometrics, and the validity of using fingerprints to identify individuals. We have thus discussed several pattern matching methods used for fingerprint recognition and developed implementations of several fingerprint recognition algorithms. We have also implemented a novel and pioneering fingerprint matching method as well as a complete fingerprint matching system with several unique and interesting properties.

In a simulated environment our NSTRA-based fingerprint recognition was capable of discriminating the source encoded tree of a highly noisy version from a database of such trees. Performing this task, it achieved an accuracy of $98 \%$. It is our opinion that if appropriate metrics about the noise inherent in a specific fingerprint system can be approximated, our NSTRA-based algorithm would be capable of providing excellent accuracy in matching even highly-fragmented fingerprints.

\subsection{Outline}

We begin the thesis, in Chapter 2, with a detailed background of the field of fingerprint recognition and an overview of the essential components which constitute a biometric system. The goal of this chapter is to provide the reader with the appropriate context and the necessary background in a concise manner.

Chapter 3 discusses the image processing aspects involved in the fingerprint identification process. The purpose of this chapter is to introduce the reader to the image processing techniques implemented and included into our fingerprint recognition system. There are a number of file format considerations, presented in Chapter 4, which were required for the construction of our fingerprint recognition system.

Chapter 5 includes both a review of several fingerprint matching algorithms of both historical and technological significance, and records the development of the implemented pattern recognition methods. The purpose of this chapter is to give 
the reader insight into the design of fingerprint recognition techniques as well as to provide a set of algorithms with which one can compare our novel pioneering fingerprint matching method.

The relevant background and implementation details of our fingerprint recognition algorithm are presented in Chapter 6. In addition, this chapter describes our testing methodology and the performance metrics of our algorithm contrasted with the algorithms described in Chapter 5.

In our concluding chapter, we summarize the findings and observations made throughout our research. A catalogue of potential future directions is also included in this chapter. 


\section{Chapter 2}

\section{Background: Survey of the Field}

It is widely believed that all humans are unique. This premise underlies the development of methods with the potential to systematically identify each and every human on the planet on the basis of biometrics such as DNA sequences, voice profiles, eye patterning and fingerprints. Collection of these measures can be made with or without our consent and used to provide evidence that we participated or did not participate in some event or that we should be included or excluded from some grouping of people. Increasing populations and global movement of people has increased the need for cheaper, faster and more accurate biometric systems. Such biometric evaluations rely heavily on advances in computer hardware and software. The aim of this thesis was to develop a complete fingerprint recognition system, and to evaluate a novel approach to matching biometric fingerprint information using a noisy subsequence tree matching algorithm. Our approach will be described following a background discussion of fingerprint biometrics.

\subsection{What is a Biometric System?}

Biometric Systems can roughly be defined as systems which use biological characteristics of individuals for some specific purpose. The most common purpose of biometric systems is to either establish or authenticate a person's identity based on the relevant 
biological characteristic. This characteristic is typically one of two types: physiological or behavioral [4].

Many different potential physiological and behavioral characteristics that could be used by a biometric system for identification or authentication purposes, have been reported in the literature. Of the two types of biometrics, the most commonlyused ones are physiological, such as DNA, ear-shape, or fingerprints. Although lesscommon, there are also many different types of behavioral characteristics that could be used for identification or authentication purposes such as signatures, keystrokes, or skill at a particular task [4].

The most common application of a biometric system is identification, although this is changing with the widespread availability of inexpensive scanners for fingerprints and the biometric software required to use them. For the purposes of this thesis "identification" refers to the determination of a person's identity from a database of identity-to-biometric-characteristic pairs. It is obvious that in the case of identification, the whole available database must be compared against the query template. As a result of the "1-to-N" comparisons that must be made, execution time is often a constraint [4].

Another application for biometric systems is authentication/verification. For the purposes of this thesis, verification or authentication refers to the verification of a claimed identity, usually for security applications. In this case, it is sufficient to achieve a comparison against only the biometric data associated with the claimed identity. Since only a single comparison is required, the corresponding execution time of the task is not usually a constraint. This allows authentication algorithms to be much more discriminating in their analysis, and consequently they can be more accurate in their claims [4]. 


\subsection{Significant Properties and Trade-offs of Bio- metric Systems}

There are some differences in the way that biometric systems operate when compared to other identification and authentication systems. While some of these differences are beneficial, others are detrimental.

Unlike security passwords and key-cards, most biometric characteristics cannot be forgotten or lost. The same phenomenon is also true of all physiological biometric characteristics. This is a significant and useful property of biometric systems when compared to their non-biometric equivalents. Another consideration of a biometric system is the monetary cost. Indeed, this is very pertinent because the scanners and software required to setup the infrastructure necessary to support a biometric system can be more expensive than that of a simpler system involving passwords or key-cards. This criterion is, however, changing since commercial fingerprint scanners are becoming more affordable, as is the software required to use them.

Most authentication and recognition systems can only operate in what is generally referred to as a "positive verification" mode. Positive recognition refers to the confirmation that a person is who s/he claims to be, or that $\mathrm{s} / \mathrm{he}$ is the person that others claim. This leads us to a common idiom or paradigm in security applications: biometric systems have the augmented benefit of being capable of operating in "positive" and "negative" verification modes. Negative recognition involves refuting the hypothesis that a person is who $\mathrm{s} /$ he claims to be, or that $\mathrm{s} /$ he is not the person who others claim $\mathrm{s} / \mathrm{he}$ is. In security applications this could be desirable since there is no way to claim that a false negative occurred due to human error [4].

There are also several different important properties of biometric systems which require consideration when they are being designed. Judging which features are required or which should be given priority is fundamental for achieving the desired results from a particular system.

These properties and trade-offs include: verification vs. identification, automated vs attended, cost and storage requirements, operating speed and accuracy, and the trade-offs between different types of biometric systems [7]. 


\subsubsection{Verification vs. Identification}

One important consideration when designing a biometric system is to determine whether the application requires identification or authentication.

Although the matching processes behind identification and authentication are often identical, the other modules of the respective systems could be very different. Identification and authentication have very different goals, and as a result, often have very different sets of constraints on the characteristics of the desired biometric system. The level of automation, quantity of storage, operating speed, overall accuracy, and the type of biometric used are all affected by the choice of whether the system is intended for identification or authentication.

Biometric systems designed for identification often have very large databases, with up to 66 million templates in the case of the Integrated Automated Fingerprint Identification System (IAFIS) [8]. Since their databases usually must be quite large, their storage requirements and the cost of storage will be correspondingly high. The storage requirements of authentication-based systems are likely to be much smaller and restricted, for example, a company desiring biometric authentication need only store enough images to account for each of their employees, or a computer user who desired biometric authentication need only store enough images to account for each of those people $\mathrm{s} /$ he wished to have access to the their personal computer.

As mentioned briefly in Section 2.1, the operating speed and accuracy requirements of identification and authentication systems are usually quite different. The type of biometric used for identification-based systems is usually one which has already been widely collected and used in previous systems, such as fingerprints. Authentication systems are, more often, able to experiment with other biometrics, and developers can therefore choose one which is the most appropriate for the specific application, as they do not have to deal with legacy issues. 


\subsubsection{Automated vs. Attended}

There are issues which must be considered when deciding which portions of the biometric system should be automated or attended/supervised. It is important to determine whether the subjects are cooperative or non-cooperative, and whether the system must operate covertly or non-covertly [7].

If the system is to be used in law enforcement, it is extremely important that it be attended, especially in the registration phase of its operation. A witness of the proper registration process is extremely important for verifying not only that official and due process was used, but also to warrant that the subject being registered has his/her identity properly represented. Another aspect of biometric systems used in law enforcement is that it must be monitored closely in the final stage of the identification process. Ultimately, a fingerprint identification expert must analyze the system's results and make the final decision, since a judge's decision in a judicial process may rest on the results of the identification process [7].

Depending on the level of security that an authentication system is designed for, whether it be for personal or corporate use, it may or may not be attended. The cost of having an attendant for the system may also come into play.

\subsubsection{Trade-off: Matching Speed vs Accuracy}

When designing a fingerprint identification or authentication system, there is always a trade-off between speed vs. accuracy. As the speed requirements of the system increases, one has to sacrifice more accuracy in order to achieve that operating speed. Conversely, as the accuracy of the system increases, the longer it will take for it to perform identification/authentication.

Redesigning the algorithm to be hierarchical in order to tune the speed and accuracy of a biometric system to the desired level, is quite common. Generally, the operation of these hierarchical strategies progress by first running less accurate but faster algorithms in order to reduce the number of candidates, and then by iteratively invoking slower but more discriminating algorithms until a threshold number of candidates is reached. 
Two terms are widely used to discriminate between different common configurations of biometric systems, namely the terms "on-line" and "off-line" [1]. These two are informally defined as follows:

- On-line: An "on-line" biometric system should be able to provide "immediate" results. It should, essentially be able to act on its own in an unattended/supervised manner. The biometrics are usually collected on site and the enrollment process could potentially be unsupervised [1]. On-line biometric systems are somewhat geared towards personal or commercial use.

- Off-line: An "off-line" biometric system is typically used when more accurate results or a higher level of security is needed. "Off-line" systems usually take a longer time to perform matching and enrollment, and often both of these tasks must be supervised due to the nature of the system's use. There may be additional restrictions on their use, e.g., high cost hardware requirements such as those which provide support for massive parallelism and storage, or manual supervision and human intervention at the final stages of identification to ensure the lowest error rate possible [1]. Off-line biometric systems are suited for use in high security and other critical applications like law enforcement.

\subsubsection{Trade-off: Biometric Types}

Other than when dealing with legacy issues, i.e., where the biometric used is virtually "cast in stone" ${ }^{1}$, choosing the type of biometric to be used is another important consideration when designing a biometric system. There are a number of characteristics commonly used to define, contrast and decide which type of biometric is pertinent when designing a biometric system. These include: universality, distinctiveness, permanence, collectability, performance, acceptability, and circumvention [1].

For the purposes of this thesis, we shall use the following definitions for these biometric characteristics [1]:

\footnotetext{
${ }^{1}$ This is due to the prohibitively large quantity of work required for replacing legacy fingerprints with another biometric type.
} 
- Universality refers to how common the biometric is amongst the population, at large. Usually, one desires a universal biometric (i.e., a biometric trait which essentially everyone possesses).

- Distinctiveness refers to the ease with which two examples of the given biometric can be distinguished from each other by a human or a machine. In other words it is a measure of the differences between two given people's biometric identifiers for the given type.

- Permanence refers to how long the biometric remains recognizable in and of itself. Normally, a relatively permanent biometric trait is desired for use in biometric systems so that stored templates do not become quickly outdated.

- Acceptability refers to how willing people are to have this biometric trait measured in daily life. A biometric system which is to be used in a non-covert manner and which must be used often and in a cooperative manner will, obviously, require a high acceptability. A system which is to be used covertly, on the other-hand, may not have to be so "acceptable".

- Circumvention refers to how easily a biometric can be copied or spoofed in order to cause a false positive response. It is obvious that the biometric chosen should not be easily circumvented.

Different levels of most of these characteristics may be desirable for a particular application. Careful consideration of what the application requires will aid in making the best decision in designing a suitable system. Table 2.2.4 (taken from [1] with some minor edits) describes the relative performance of several different common (and a few uncommon) biometric types with regard to each of the characteristics described in this section.

It is evident from Table 2.2.4 that fingerprints are an excellent choice when designing biometric systems. This assertion will be discussed in greater detail below. Other reasonable choices include irises and retina. 


\begin{tabular}{llllllll} 
& \\
Biometric & $\mathrm{H}$ & $\mathrm{H}$ & $\mathrm{H}$ & $\mathrm{L}$ & $\mathrm{H}$ & $\mathrm{L}$ & $\mathrm{L}$ \\
\hline DNA & $\mathrm{M}$ & $\mathrm{M}$ & $\mathrm{H}$ & $\mathrm{M}$ & $\mathrm{M}$ & $\mathrm{H}$ & $\mathrm{M}$ \\
Ear Shape & $\mathrm{H}$ & $\mathrm{L}$ & $\mathrm{M}$ & $\mathrm{H}$ & $\mathrm{L}$ & $\mathrm{H}$ & $\mathrm{H}$ \\
Face & $\mathrm{H}$ & $\mathrm{H}$ & $\mathrm{L}$ & $\mathrm{H}$ & $\mathrm{M}$ & $\mathrm{H}$ & $\mathrm{L}$ \\
Facial Thermogram & $\mathrm{M}$ & $\mathrm{H}$ & $\mathrm{H}$ & $\mathrm{M}$ & $\mathrm{H}$ & $\mathrm{M}$ & $\mathrm{M}$ \\
\hline Fingerprint & $\mathrm{M}$ & $\mathrm{L}$ & $\mathrm{L}$ & $\mathrm{H}$ & $\mathrm{L}$ & $\mathrm{H}$ & $\mathrm{M}$ \\
\hline Gait & $\mathrm{M}$ & $\mathrm{M}$ & $\mathrm{M}$ & $\mathrm{H}$ & $\mathrm{M}$ & $\mathrm{M}$ & $\mathrm{M}$ \\
Hand Geometry & $\mathrm{M}$ & $\mathrm{M}$ & $\mathrm{M}$ & $\mathrm{M}$ & $\mathrm{M}$ & $\mathrm{M}$ & $\mathrm{L}$ \\
Hand Vein & $\mathrm{H}$ & $\mathrm{H}$ & $\mathrm{H}$ & $\mathrm{M}$ & $\mathrm{H}$ & $\mathrm{L}$ & $\mathrm{L}$ \\
Iris & $\mathrm{L}$ & $\mathrm{L}$ & $\mathrm{L}$ & $\mathrm{M}$ & $\mathrm{L}$ & $\mathrm{M}$ & $\mathrm{M}$ \\
Keystroke & $\mathrm{H}$ & $\mathrm{H}$ & $\mathrm{H}$ & $\mathrm{L}$ & $\mathrm{L}$ & $\mathrm{M}$ & $\mathrm{L}$ \\
Odor & $\mathrm{H}$ & $\mathrm{H}$ & $\mathrm{M}$ & $\mathrm{L}$ & $\mathrm{H}$ & $\mathrm{L}$ & $\mathrm{L}$ \\
Retina & $\mathrm{L}$ & $\mathrm{L}$ & $\mathrm{L}$ & $\mathrm{H}$ & $\mathrm{L}$ & $\mathrm{H}$ & $\mathrm{H}$ \\
Signature & $\mathrm{M}$ & $\mathrm{L}$ & $\mathrm{L}$ & $\mathrm{M}$ & $\mathrm{L}$ & $\mathrm{H}$ & $\mathrm{H}$ \\
Voice & $\mathrm{M}$ & & & & & \\
\hline
\end{tabular}

Table 2.1: Comparison of example biometrics by characteristic, included from "Handbook of Fingerprint Recognition" [1].

\subsection{Why Fingerprints are Effective Biometrics}

As argued in the literature, fingerprints are an excellent choice for a differentiating characteristic in a biometric system. There are several applications of biometric systems which could only work using fingerprints, and which would not be viable with any other biometric (due to legacy issues).

In this regard, as shown in Table 2.2.4, fingerprints earn a score of high when it concerns: distinctiveness, permanence and performance. Fingerprints also score well in all the other categories.

Every individual has fingerprints except for those who have severely-damaged fingers or certain genetic defects [9]. Over time, fingerprints have been shown to be relatively distinct as no two identical/indistinguishable fingerprints have ever been discovered, there also exist several models of the individuality of fingerprints which show they are more than suitable for identification purposes $[4,10]$ (fingerprint distinctiveness is discussed in more detail in Section 2.4). Fingerprints are permanent 
unless the finger(s) have been damaged to the point that they will not heal; damaged fingerprints may also possess sufficient information for identity differentiation.

There are numerous ways to collect fingerprints, and many modern techniques only require that a finger be pressed against a sensor which prevents the need to use the traditional ink-and-paper family of fingerprint collection methods, which are often unpleasant. Many of the fingerprint-based biometric systems in use today are extremely efficient, and can offer results in seconds (except in special cases like the IAFIS which takes 10 minutes on average to retrieve results [8]).

One unfortunate aspect of using fingerprints is that there is a stigma associated with their use. Because of their long history of use in law enforcement, people usually associate having their fingerprints impressions collected with being treated as a criminal. A common complaint from individuals using systems which require fingerprints being taken is that they feel like their privacy has been violated or that they are being treated like criminals $[11,12,13]$. This taint of criminality is less evident in other biometric systems such as retinal or iris scans which are increasingly used in airports.

Another unfortunate issue with using fingerprints in security (verification) is that security measures built on fingerprints are relatively easily circumvented. If fingerprints from an individual can be obtained, with the proper "tools", there is nothing stopping a person from accessing a system secured solely through fingerprints. However, by combining fingerprints and some other type of verification, an acceptable level of security can be reached [14].

There are several other acceptable choices for a biometric, as can be seen from Table 2.2.4. Irises, ear shapes, or hand geometry would all be acceptable as biometrics, although none of them have been as widely studied as fingerprints.

\subsection{Fingerprint Distinctiveness and Validity/Ac- curacy as a Biometric}

The admissibility of fingerprint evidence in court is something that is generally accepted today, although there has been some contention in the past. 
In order for fingerprints to be admissible for identification purposes in a courtroom, the court requires that there has to be some proof of the "reliability" of fingerprints as evidence as well as a notion of its "accuracy". The kind of "reliability" considered in courtrooms is not equivalent to the definition of "reliability" used by the scientific community [15]. Rather, a courtroom's definition of "reliability" is closer to the scientific definition of "validity" [15]. In order to ensure clarity the following definitions of accuracy, reliability, and validity will be used in this work:

- Reliability is the repeatability and consistency of an experiment.

- Validity is a measure of how fairly an experiment tests its hypothesis.

- Accuracy is the difference between the experimental result and the "true" accepted value. The stated uncertainty in an experimental result should always be greater than this percentage accuracy. Accuracy is also associated with the inherent uncertainty in a measurement.

To summarize, in order for a fingerprint-based identification scheme to be used in court, one must demonstrate that the identification is both "valid" and "accurate" [15].

Fingerprints have been used for identification for centuries [4]. The validity of fingerprint-based identification is connected to the uniqueness of fingerprints. Although it is impossible to prove that all fingerprints are unique and distinguishable [15], as mentioned above it can be empirically shown that it is valid for identification, since no two identical or indistinguishable fingerprints have been discovered [10]. The accuracy of fingerprint identification techniques, however, has been repeatedly called into question in recent years [15]. No study on the accuracy of the entire process of fingerprint identification has been reported, therefore, the accuracy of modern fingerprint identification systems is unknown. Numerous studies of the accuracy of the automated portion of fingerprint identification processes have been studied, the portion that remains unstudied is the manual examination by expert witnesses. The study of the accuracy of a full and applied fingerprint identification system is outside 
the scope of this thesis ${ }^{2}$.

\subsubsection{Admissibility in Court}

In the past, the admissibility of the match of a particular fingerprint as evidence in court was decided by the number of minutiae which were successfully matched. The requirement on this number was that it had to be at least between 8-12 There were issues with this approach however, as the number of minutiae matched is not directly correlated with the quality of the fingerprint matching phenomenon itself. The quality of the area of the fingerprint image surrounding each minutiae is more representative of the validity of each minutiae match, and consequently, of the fingerprint matching itself. If the fingerprint image is of low quality, it will also be difficult for the human supervisor to determine with certainty whether or not the match is correct. Fingerprint experts instead base the admissibility of the match on several qualitative properties of the area surrounding the matched minutiae such as smudging, inconsistencies in contrast, contaminants and other similar sources of noise [1].

\subsection{Biology of Fingerprint Uniqueness}

The pattern of ridges on the tips of our fingers defines our fingerprints. Formation of fingerprint patterns have a genetic component, as evidenced in individuals with genetic defects in epidermal ridge formation [9], but are strongly influenced by environmental factors. Even identical twins who by definition share identical genomic DNA sequences, possess fingerprints which bear similarity but not identity [16]. Fingerprints are fully formed prenatally and are retained throughout life. Although there is some influence of genetics on overall patterning, environmental factors play a key role in establishing the final patterns that an individual will carry throughout their lifetime. The key characteristics of fingerprints first emerge when the fingertips begin

\footnotetext{
${ }^{2}$ In spite of this, it is fair to mention that the accuracy of an automated portion of a fingerprint identification system is discussed here.
} 
to form during embryonic gastrulation. It is believed that the unique microenvironments both surrounding and within each digit influence the process of local epidermal cell proliferation and migration. Ridge pattern formation is therefore the culmination of many distinct local factors and forces which over the course of embryonic development are unique from digit to digit. Thus, the digits of each identical twin are exposed to unique environments and therefore exhibit unique microdetails in fingerprints which allow for discrimination between them (see [1], and references therein).

The modern forensic use of fingerprints to identify individuals began in the 19th century $[17,18]$ although as previously mentioned there are a number of reports suggesting the awareness of fingerprint uniqueness many centuries previous [3, 19]. There are also many modern models of fingerprint uniqueness which can be used to show that they are suitable for identification [10].

\subsection{The Fingerprint Identification Process}

All biometric systems follow a similar pattern in their construction and organization. This is true for the phases up to and including the process by which they identify or authenticate individuals.

The process begins with the data collection of raw biological data from the subject(s). This includes pre-processing of the raw data (if necessary). This is followed by the extraction of the useful features and the construction of the template. Matching based on the templates obtained from feature extraction is then involved. The final tasks include the manual ${ }^{3}$ auditing of the results, and registration ${ }^{4}$.

\subsubsection{Raw Data Collection}

Collection of raw fingerprint data has changed significantly over time, starting from simpler ink-based collection techniques to the use of much more convenient and less error-prone fingerprint scanners. Although the standard techniques for fingerprint

\footnotetext{
${ }^{3}$ In most cases, identification systems are checked manually by an expert so as to ensure that no mistakes have been made, or to perform the final consolidation step.

${ }^{4}$ This phase is outside the scope of this thesis.
} 
collection have changed greatly, the use of older fingerprints in newer systems is a necessity for several applied biometric systems. This is because fingerprints that were collected using older techniques must still be used in some systems, and thus it is important that the issues that are present in these collection techniques be taken into consideration.

More recent collection techniques center around the concept of electronic fingerprint scanners. These scanners use a wide variety of sensors, but all of them subsequently reduce the scanned fingerprint to an image of some kind. The primary purpose of fingerprint scanners is to collect fingerprints. However, there are several electronic fingerprint scanners that include extra hardware which is capable of performing different processing tasks up to and including matching [1].

\section{Important Fingerprint Scanner Characteristics}

In order to regulate the quality of fingerprint images produced by fingerprint scanners and other fingerprint collection techniques, the FBI has set several restrictions on the images produced by a fingerprint scanner. Only a scanner possessing these properties can be used in their systems; and they in turn should have stringent requirements with respect to geometric accuracy, fingerprint gray range, fingerprint artifacts and anomalies, and fingerprint sharpness and detail rendition.

A summary of the requirements placed on the 500DPI fingerprint images [20] is given below:

- Geometric Accuracy: The difference, $\Delta$, between the actual value and the measured value of distance between the test set of scanning material should not exceed:

$$
\begin{aligned}
& \Delta \leq 0.0007, \text { for } 0.00<X \leq 0.07 \\
& \Delta \leq 0.01 X, \text { for } 0.07 \leq X \leq 1.50
\end{aligned}
$$

where:

$$
\Delta=|Y-X|,
$$




$$
\begin{aligned}
& X=\text { actual target distance, } \\
& Y=\text { measured image distance, } \\
& \Delta, \quad X, \quad Y \text { are in inches. }
\end{aligned}
$$

- Fingerprint Gray Range: For the majority of the produced images, there should be at least 200 gray-levels (before the conversion to a digital image).

- Fingerprint Artifacts and Anomalies: Artifacts or anomalies produced by the scanner must not have a significant adverse affect on the corresponding matching systems.

- Fingerprint Sharpness and Detail Rendition: The sharpness and detail of the fingerprint images should be high enough to support conclusive fingerprint comparisons, fingerprint classification, automatic feature detection, and the overall Integrated Automated Fingerprint Identification System.

\section{Off-line Fingerprint Sensing}

Off-line fingerprint sensing includes the "ink-technique" as well as the collection of latent fingerprints. The most commonly used technique for fingerprint collection in law enforcement is known as the "ink-technique" [21,22]. This technique is being replaced by electronic scanners over time, but it is still used today [1]. As a result, automated fingerprint identification systems must be able to handle both fingerprints collected using the "ink-technique" style and those collected by electronic scanners.

We summarize below the procedures for collecting fingerprints via the "ink-technique" [8]:

1. The subject is to wash his/her hands with soap and water or rubbing alcohol;

2. The height for recording fingerprints is approximately 39 inches $(99.06 \mathrm{~cm})$ from the floor.

3. The subject must be instructed to look away from the fingerprint device, and not to assist in the process. 
4. The fingerprint pattern areas must be covered evenly with ink.

5. Each finger on the collection paper must be rolled from "nail-to-nail".

6. The paper must later be scanned and stored in a database.

Ink-based techniques are susceptible to error, and require great skill to perform properly and without incident [8]. There are obvious advantages to developing fingerprint collection techniques which do not require training as extensive or as specific as that required for ink-based techniques.

In addition to fingerprint collection in a controlled environment, collection techniques of latent fingerprints are extremely important, and is a subject of much research. As there are a wide variety of possible surfaces where the fingerprints could be located, there are also a wide variety of techniques for latent fingerprint extraction from various substrates.

Latent fingerprints are the result of oil and moisture on the fingertips, which, in turn, leave residue on various objects, which can be analyzed to get an impression of a fingerprint. These fingerprint impressions are of notoriously low quality, and as a result often require clever techniques to enhance their impression. Common techniques for latent fingerprint collection include dusting, ninhydrin spraying, iodine fuming, and silver nitrate fuming [21, 22].

\section{On-line Fingerprint Sensing}

On-line fingerprint sensing primarily consists of fingerprint scanners. These scanners, usually, follow a pattern in their construction. All fingerprint scanners have a sensor for collecting the analog data, and a method of converting this data into digital information (see Figure 2.1).

Fingerprint scanners today most often operate using the principle of Frustrated Total Internal Reflection (FTIR). FTIR is also one of the oldest techniques for on-line fingerprint collection [23].

A glass prism is the surface touched by the finger. As a result of this, diffused light is emitted from one side of the prism and reflected or absorbed by the fingerprint's 


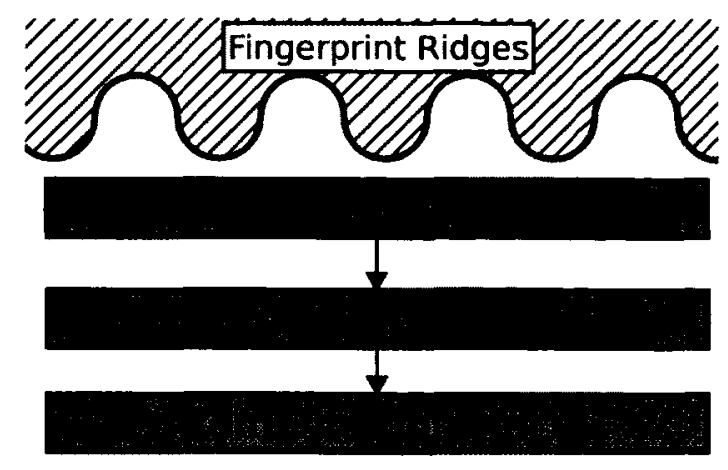

Figure 2.1: General structure of a fingerprint scanner. Drawn from a description reported in [1].

ridges and valleys. The ridges which are in contact with the prism have the effect of scattering light that makes contact with it. The valleys, which are slightly raised from the prism when appropriate pressure is used, have the effect of reflecting the light back to a sensor. In this way ridges and valleys can be distinguished from each-other. The sensor used could be any device similar to a Charge-Coupled Device (CCD) or a Complementary Metal-Oxide-Semiconductor (CMOS). Since this method mandates a good contact between the finger and the prism, dry fingers can cause issues. Another problem with this technique is the geometric distortion it creates since the sensor will not always be parallel with the finger surface [1,23].

Since the FTIR technique depends on a three dimensional impression, it has some security benefits when it concerns attacks which utilize two dimensional copies of the fingerprints [1].

\subsubsection{Data Enhancement}

Fingerprint identification, both manual and automated, depends on the quality of the image when it concerns performance ${ }^{5}$. There are many different kinds of noise that can cause issues when performing fingerprint matching. These include: rotation, displacement, distortion due to skin plasticity, variance in pressure when the

\footnotetext{
${ }^{5}$ Performance here refers to how discriminating a system can be about a given fingerprint image.
} 
print is taken, use of older fingerprint images, and differences in fingerprint collection techniques.

There are a number of pre-processing techniques reported in the literature that can be used to improve the relative quality of the raw fingerprint data so that the relevant important features are more easily extracted. These types of noise could include pixel-damage, inconsistent contrast, discontinuities in ridges, and the lack of depth in the gradient of the intensity between the ridges and valleys [4].

The different techniques that are often used to enhance a fingerprint image before it is used by a matching algorithm include Gaussian blurring, contrast adjustment, and the binarization of the image (using histogram analysis and thresholding). These are explained in more detail in Chapter 3.

\subsubsection{Feature Extraction and Template Construction}

Feature extraction is the process of extracting useful features for identification and/or authentication from the biometric. This phase is tied to the process of image enhancement, and it is difficult determine where the image enhancement process ceases and the feature extraction begins [1]. The steps involved in feature extraction, especially when it concerns minutiae, almost always involves all or a subset of the following as outlined in [1]:

- Orientation estimation.

- Frequency estimation.

- Segmentation of the image into similar regions.

- Ridge detection and thinning.

- Minutia detection.

Orientation estimation, frequency estimation, ridge detection and thinning, and image segmentation are also used in many types of fingerprint image enhancement modules. This is what makes the distinction between the two stages difficult $[24,25]$. 
The feature most commonly extracted from fingerprints for analysis are the socalled minutiae or singular points (see Figure 2.3). The exact definition of the term "minutia" differs across the literature but it is most often used to refer to several different types of ridge features in addition to a set of associated metrics. There are three types of minutiae typically extracted by minutia-extraction algorithms. These minutiae are known as ridge bifurcations, ridge endings and ridge islands or dots. Minutiae are usually represented using their Cartesian coordinates, along with some additional relevant data, for example, a quality score, the angle of the most closelyassociated ridge, and the minutia type ${ }^{6}$. Minutia are typically stored as a 3-tuple, $(x, y, \theta)$, where $x$ and $y$ are the Cartesian coordinates, and $\theta$ is the angle of the most closely-associated ridge to the given minutia with the horizontal axis.

Most methods of minutia-extraction follow a similar pattern involving an orientation estimation, segmentation of the fingerprint image, ridge detection and thinning, and finally, the minutia detection itself.

\section{Orientation Estimation}

This phase of the minutia-extraction process is used later to determine the $\theta$ value of each minutia and is used in several different minutia-extraction algorithms. The fingerprint image is typically separated into small regions and the gradient is analyzed to estimate the average direction of the ridges contained within that particular section. In order to make the estimate as accurate as possible the regions can be reduced in size [26].

A simple method for accomplishing the analysis of the gradient in each region is by sampling a set of pixels and determining the gradient at each pixel's location. Once the gradient has been determined, the direction of the ridges should be perpendicular to the angle of the gradient [1].

One example of such a method is reported by Ratha, Chen and Jain in [26]. The method is founded on the expressions given by Eq. (2.1)-(2.4).

\footnotetext{
${ }^{6}$ Many other pieces of information are used by various minutia-matching methods, but these are by far the most common [1].
} 


$$
\begin{aligned}
\theta_{i j} & =90^{\circ}+\frac{1}{2} \arctan \frac{2 G_{x y}}{G_{x x}-G_{y y}} \\
G_{x y} & =\sum_{h=-8}^{8} \sum_{k=-8}^{8} \nabla_{x}\left(x_{i}+h, y_{j}+k\right) \cdot \nabla_{y}\left(x_{i}+h, y_{j}+k\right), \\
G_{x x} & =\sum_{h=-8}^{8} \sum_{k=-8}^{8} \nabla_{x}\left(x_{i}+h, y_{j}+k\right)^{2}, \\
G_{y y} & =\sum_{h=-8}^{8} \sum_{k=-8}^{8} \nabla_{y}\left(x_{i}+h, y_{j}+k\right)^{2} .
\end{aligned}
$$

\section{Frequency Estimation}

Frequency estimation deals with the task of determining the approximate frequency of the ridges in a certain region. Several different techniques make use of this information including the Gabor filtering process described in Section 2.6.2.

There are a number of ways for estimating the frequency of the ridges. One simple method is to estimate the average number of pixels in between ridge peaks. This can be accomplished by using oriented windows as in Figure 2.2. Using such an oriented window, the pixels between the ridge peaks can be counted, and the ridge-frequency of the region estimated [2]. A "signature" of the ridges and valleys, $X$, is obtained by using Eq. (2.5); if there are no minutiae or singular points within the region, the "signature" will have the same frequency as the region's ridge frequency [2]. Let $G$ be the normalized image generated from the original fingerprint image, $O$ be the orientation image generated from a process like that described above, $x$ and $y$ be the coordinates within the oriented window, and $i$ and $j$ be the coordinates of the central pixel of the given orientation window. From $X$ the approximate ridge-frequency of the local region can be estimated.

$$
X[k]=\frac{1}{y} \sum_{d=0}^{y-1} G(u, v)
$$

where: 


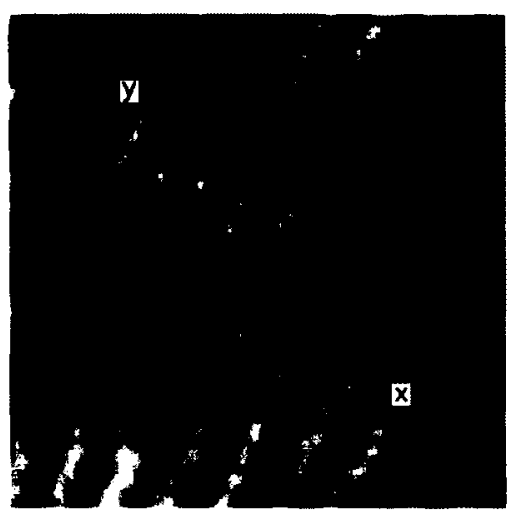

Figure 2.2: Illustration of an orientation window on a group of ridges with similar $\theta$ values. Diagram similar to that in [2].

$$
\begin{aligned}
& u=i+\left(d-\frac{y}{2}\right) \cos O(i, j)+\left(k-\frac{x}{2}\right) \sin O(i, j) \\
& v=j+\left(d-\frac{y}{2}\right) \sin O(i, j)+\left(\frac{x}{2}-k\right) \cos O(i, j)
\end{aligned}
$$

\section{Segmentation of the Image}

Most fingerprint images will have areas which are not related to the fingerprint itself, and instead, consist of blank space containing only noise. The noise could be accumulated from several different sources: from dust, grease or from other contaminants that have been left behind on the fingerprint scanner from previous subjects. Besides these, other areas can be damaged or be highly distorted portions of the fingerprint impression itself, which are rendered unusably noisy as a result of smudging or improper pressure. In order to prevent the collection of erroneous minutiae, it is obviously desirable to mark off these sections which are too noisy or which are not part of the fingerprint so that they may be ignored. The areas which do not contain any useful information are generally referred to as the "background", and the areas which contain useful fingerprint ridges are referred to as the "foreground" [2].

It is difficult to separate the foreground from the background by using only pixel intensity and thresholding, since fingerprints themselves are striated patterns [27]. Because of noise, it may also be difficult to distinguish the areas which do not contain fingerprint ridges from the foreground. 
One successful technique for separating the foreground from the background was presented by Mehtre et al. [28]. This technique is based on achieving a segmentation of the fingerprint image using the ridge orientations. By mapping locations based on their ridge orientations, and by also using the histogram of the orientations, the foreground can be distinguished by analyzing the peaks in the histograms.

Segmentation can also be achieved through another technique proposed in [26]. This method is able to segment the fingerprint image based on the so-called "areas of interest". The algorithm is capable of determining which regions contain high noise and/or which areas lack fingerprint ridges ${ }^{7}$ and to thereafter mask them. This is accomplished by computing the variance in gray levels in the direction perpendicular to the orientation field in each region or block that is visited. The "areas of interest" are then the regions or blocks with the highest variance, where this conclusion is based on the assumption that in any "areas of interest", there will be a great variance in the pixel intensity in the direction perpendicular to the dominant ridge orientation.

\section{Ridge Detection/Thinning and Minutia Detection}

The final step in the minutia-extraction process is to perform the extraction itself. Several different types of minutiae can be found in fingerprints, and the three most common types are illustrated in Figure 2.3.

A common technique for minutia-extraction is the reduction of the remaining fingerprint image to a skeletal representation, which, in turn, is achieved by finding the peaks of the gradients between the ridges, and by marking them as the pixels which represent the ridge-peaks [29]. By keeping only those pixels which represent ridge-peaks, one can obtain an image which contains thinned-out representations of these ridges. Once there are only single pixel wide representations of the ridges, simple and straightforward minutia detection techniques can be used on the remaining image skeleton. Although, at this stage, minutia detection can be performed, we believe that filtering should be performed so as to remove any of the spurious ridges created from the process of reducing the image to its skeleton [2]. Finally, once the image has been appropriately filtered, the minutia detection can be performed.

\footnotetext{
${ }^{7}$ These regions are the empty regions or regions with only smudges and blurred areas.
} 

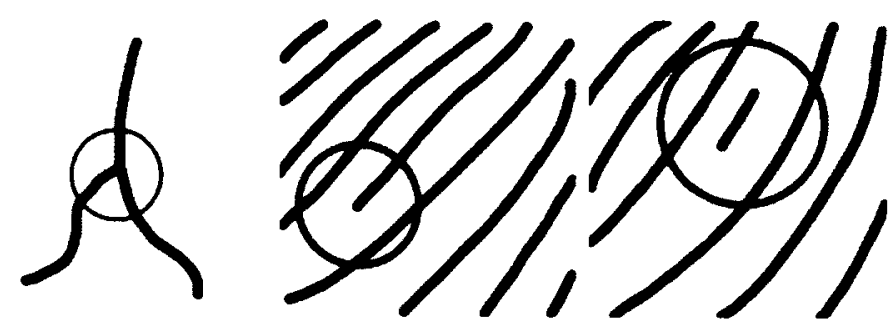

Figure 2.3: The different types of minutiae. From left to right, are the ridge bifurcation minutia, the ridge-ending minutia, and the short ridge minutia.

A common and simple technique for minutiae detection involves iterating through each ridge pixel of the skeletal representation and checking each of the pixel's eight adjacent neighbors for other ridge pixels. If there are three ridge pixels, then the pixel is a bifurcation type minutia. If there is only a single adjacent ridge pixel, then the pixel is a ridge-ending type minutia [30]. The third type of minutia, the short-ridge or island minutia is typically ignored or detected through a later process. Island minutiae are often ignored due to their noisy nature, their proper detection can be adversely affected even through small variances in pressure applied by a subject as their finger is placed on a fingerprint sensor [4].

\subsubsection{Minutia Matching}

Once all the required features have been extracted, matching can be achieved. Matching algorithms are broad and varied in their approaches, techniques, and methodologies, and employ many different strategies in an attempt to increase their efficiency, to increase their match-speed, to reduce the memory footprint, or to improve accuracy.

There are two rough groups of matching algorithms based on the scope of their respective matching techniques. These two groups are commonly referred to as "global matching techniques" and "local matching techniques". There are significant differences in the way these two types of matching algorithms are typically designed, in what contexts they are used, and how they treat or process their data. The trade-off's between local and global techniques include: algorithm complexity, computational complexity, distortion tolerance, and discriminatory power. 


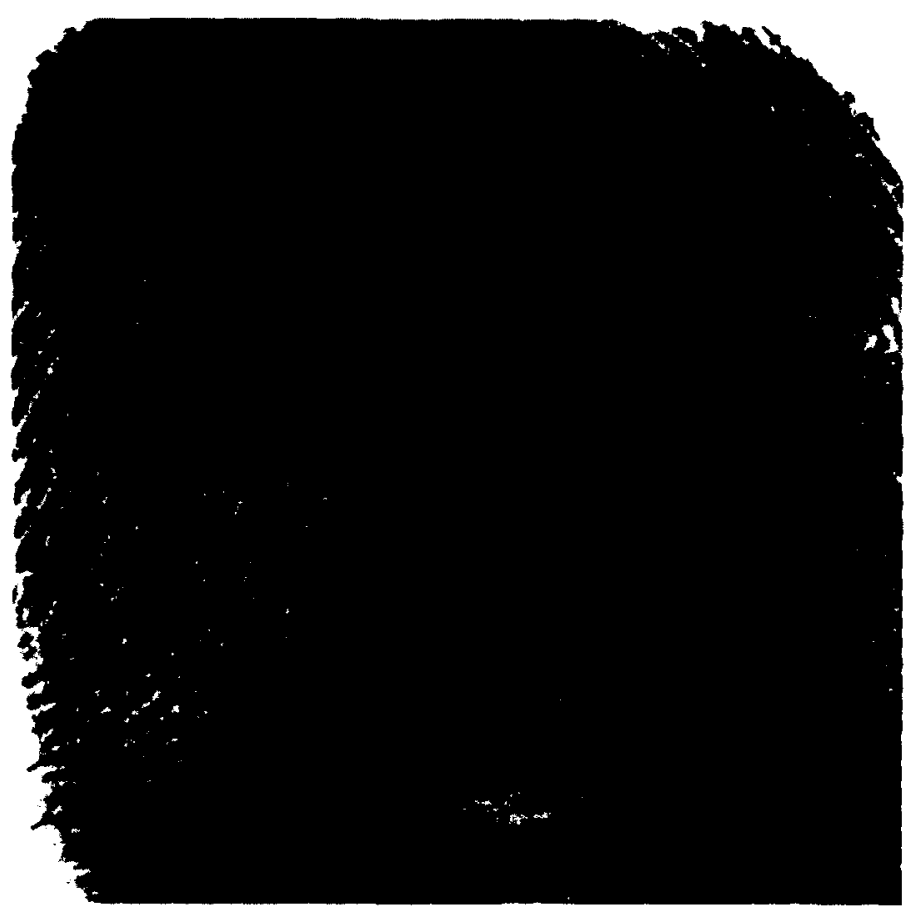

Figure 2.4: The minutiae on this fingerprint have been highlighted with red squares, the blue line indicates the direction of the most closely associated ridge, this is known as the minutia's $\theta$ value. 


\section{Global Schemes}

Global techniques favor viewing the fingerprint as a whole by taking into account all the global-spatial relationships between fingerprint features. Because the globalspatial relationships hold a great deal of valuable distinctive information, it is clear that global techniques are capable of greater discrimination between fingerprints [4]. A disadvantage of such global techniques, however, is that the same features that lead to the global techniques providing improved accuracy and discrimination capabilities, also give them a greater sensitivity to noise. Global spatial features and structures are more affected by noise than the smaller and more local structures and features which are used in locally-oriented algorithms [1].

Many global algorithms go through an extensive and detailed alignment process in order to align the minutiae as closely as possible. This is because these algorithms are often rotation dependent.

\section{Local Schemes}

Local techniques favor viewing the fingerprint as a group of localized structures or clusters of structures. Local techniques, typically, differ in the way in which they encode the information contained within local minutiae into structures. Local matching works on the principle of testing for the presence of these different localized structures. Although the structures themselves may not be unique to a particular fingerprint, a particular combination of those structures may be. As a consequence of the nature of local matching, more fingerprint images are usually required to match one physical fingerprint to another. Another technique for ensuring accuracy and the elimination of false positives (which are common in local matches) is a consolidation step [1, 25].

As a result of discarding the global-spatial relationships in favor of localized ones, local matching algorithms are less sensitive to various types of noise (e.g., displacement, and distortion due to skin plasticity). Another result of eschewing global-spatial relationships is the potential for creating rotationally independent algorithms. Indeed, several of these algorithms have been reported in the literature [1]. 


\subsection{Non-Minutia-based Matching Techniques}

In addition to minutiae, other features present in fingerprints have been used for identification and authentication purposes. The different features of fingerprints have been roughly divided into three levels [31]. The first level of features encompass the ridge flow and the pattern shape of the fingerprint. The second level contains the minutiae. The third level includes all the remaining attributes of the ridges (e.g., width, shape, edge contour, pores, and scars).

Minutiae have been studied in great detail in the literature and have been the cornerstone of research in fingerprint-based identification and authentication [4], but the third level features have been less popular as a subject of study due to the stringent requirements on the level of detail required in the image (usually greater than 1,000 DPI) [31]. In spite of this, less popular algorithms, which make use of level three features, do exist in the literature.

Pores have gained some interest in recent years as being features with which one can discriminate fingerprints. The purpose of the pores on the finger are primarily to excrete oil and sweat. They are present only on ridges and appear either open and closed. Pores will not always be detected by scanners because of the varying level of pressure applied by the subject. This results in an inconsistent number of pores between fingerprint scans, which, obviously, poses a problem for matching using pores [31].

As a feature for fingerprint identification they can be used to improve the discriminatory power of a fingerprint system. However, as with other level three features they require a higher resolution fingerprint image than is standard ${ }^{8}$ in order to be properly extracted.

One of the early techniques which used pores and other level three features for identification/authentication was presented in [31]. Pore extraction was performed by using a Gabor filter to extract only the ridges and to fill in all the pores. Once extraction has been completed the Gabor-filtered image was added to the original to allow for the use of a band pass filter to collect the high negative frequency response

\footnotetext{
${ }^{8}$ The most common format for fingerprint images is 500 DPI.
} 
of the change of the intensity of the pores. The frequency response is obtained using a Mexican Hat wavelet transform. Once the pores are extracted, matching proceeds in much the same manner as with minutia matching but with extra measures to handle the unique characteristics of pores [31].

\subsubsection{Refining Results}

Biometric identification systems like those used by the FBI and RCMP have very large databases $[1,8]$ and because of the nature of identification, "1-to- $N$ " matches are required. Each fingerprint matching process is inherently independent, and it is obvious that the identification process's computation time grows linearly with the size of the database.

In order to compensate for the massive amount of computation required for the equally massive databases of the FBI and RCMP, there are several techniques which can be exploited to reduce the amount of computation required. In addition, most of the steps in fingerprint matching can be performed in advance of a query template. As a result of this independence, it is obvious that the fingerprint matching process is trivially parallelizable. A trade-off between the overall system cost and its matching speed is a truism, permitting nearly arbitrary speed without the need to sacrifice accuracy ${ }^{9}$.

Many systems use a hierarchical approach to matching in order to reduce the amount of computation required. Using level one features, such as fingerprint patterns (e.g., loop, and whorl), allows for the quick elimination of highly dissimilar fingerprints to that of the template, which can greatly reduce the amount of processing necessary [4].

\subsection{Chapter Summary}

In this chapter we have discussed a variety of topics related to fingerprint matching in order to give context to the remaining chapters of this thesis. We have discussed:

\footnotetext{
${ }^{9}$ This, of course, implies "infinite" resources too!
} 
- Generic biometric systems and their relationship to fingerprint matching systems.

- Important points of consideration when designing a biometric system.

- Why fingerprints are an appropriate choice for biometric systems.

- The validity of using fingerprints for identification.

- The biological aspects of fingerprint uniqueness.

- The Fingerprint matching process.

Knowledge of these areas is essential for building the necessary context with which to understand and appreciate the remaining chapters. 


\section{Chapter 3}

\section{Image Processing Aspects}

Image processing is fundamental to the fingerprint matching process, and is discussed briefly in Chapter 2 (Section 2.6.2).

The ultimate goal of image processing, when it concerns fingerprint identification/verification, is the improvement of the overall quality of the input fingerprint images. Ideally, an image processing phase would be able to take a very low quality fingerprint and output a high quality one. The damage/noise that is typically present in fingerprint images includes the following types [1]:

- Breaks in the continuity of the ridges.

- Shallow gradient between the ridges (caused by smudging or otherwise).

- Damage to the finger itself through cuts, creases, burns, and bruises.

- Damage or noise added through the measurement equipment itself.

Some of these are obvious to the human eye, and unsurprisingly, fingerprint experts are able to repair "recoverable regions" of fingerprints by invoking fingerprint imagepreparation programs [1]. This has been used to support the argument that these "recoverable regions" should be detectable and repairable by a machine [1]. Areas which cannot be repaired can be removed or "masked" using segmentation techniques as detailed in 2 (Section 2.6.3). 
The methods used to cope with such damage/noise include several different filtering techniques. Some of the more common techniques for fingerprint image enhancement will be discussed in Section 3.2 including Gaussian blurring, contrast adjustment, Gabor-based filtering, and fingerprint image binarization. Beyond the techniques covered in Section 3.2, many schemes for image enhancement exist in the literature, which can be used in an attempt to improve the quality of the fingerprint images themselves.

Due to the greatly varying nature in which minutia matching algorithms operate, image enhancement techniques which work well for a given matching algorithm may not provide the same level of enhancement, for other matching algorithms. Rather, they may even reduce the effectiveness of some matching techniques due to the loss of certain features [1]. This will become increasingly pronounced as the use of features other than minutiae become widespread.

In the following chapter, we will discuss the image processing techniques implemented for use in the developed system, all of which were for adding artificial noise. We will also discuss methods of image enhancement commonly used in fingerprint matching systems. Subsequently we will briefly discuss the additional tools used in the developed system.

\subsection{Adding Artificial Noise}

For the purposes of testing the resistance to noise of the implemented algorithms, three separate algorithms for adding noise were developed: an algorithm providing two-dimensional convolution with an arbitrary square kernel, an algorithm providing Gaussian additive noise, and finally, an algorithm providing randomized pixeldamage. Each of these noise types are displayed in Figure 3.1. Any of these techniques can be used to apply noise before executing a matching algorithm to test for its sensitivity to noise. 


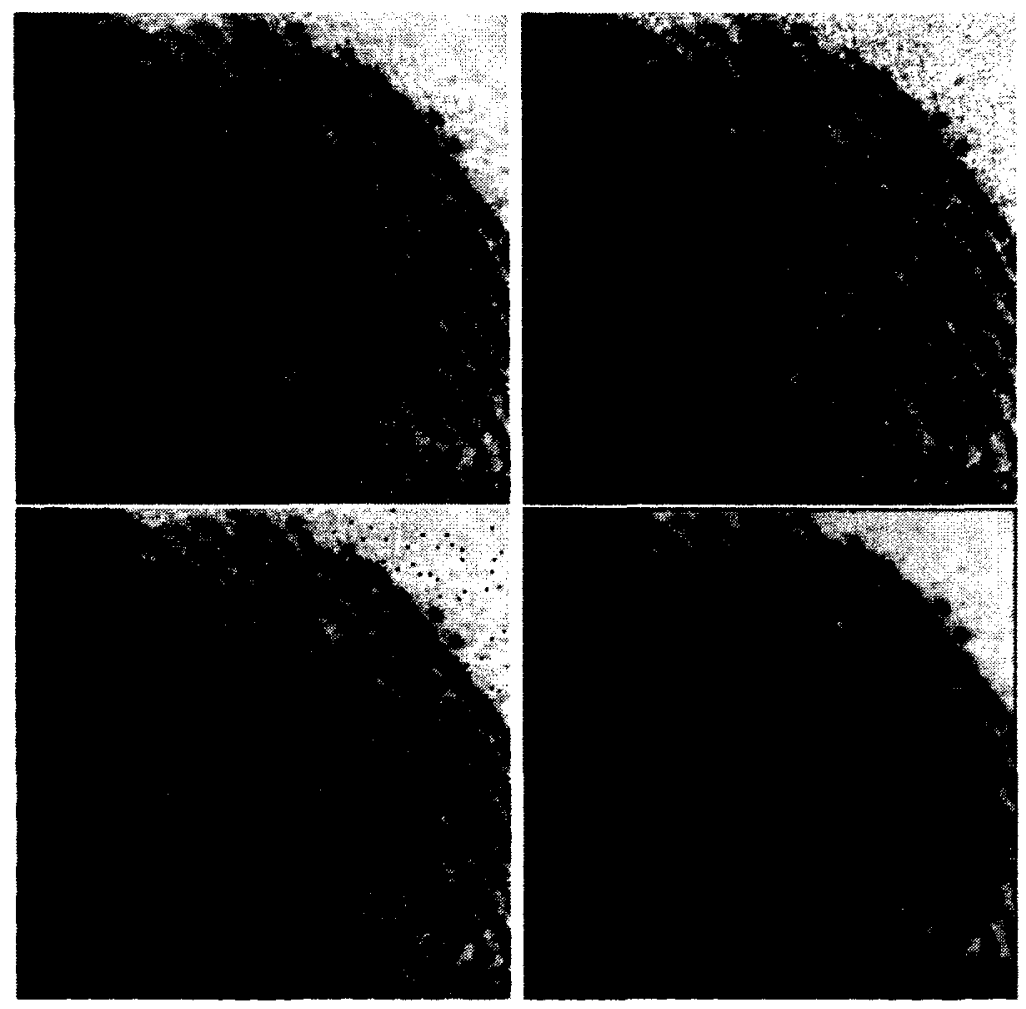

Figure 3.1: Examples of the different types of noise which can be applied. From left to right and top to bottom, we have the original image, the image with Gaussian additive noise applied, the image with pixel damage (salt and pepper) noise applied, and finally with Gaussian blur (convolution with a Gaussian Kernel) applied. 


\subsubsection{Convolution with Arbitrary Kernels}

Convolution is a powerful technique in image processing/manipulation. With the aid of a diverse set of kernels, convolution can provide a variety of different effects on the target image.

The convolution technique is summarized in Algorithm 1. This is an overlysimplified version of the actual algorithm used which, while optimized, is quite complex for illustrative purposes. The algorithm uses the input array of the raw image data $\mathcal{A}$, and the input array of the kernel data $\mathcal{K}$, and processes them to perform the convolution.

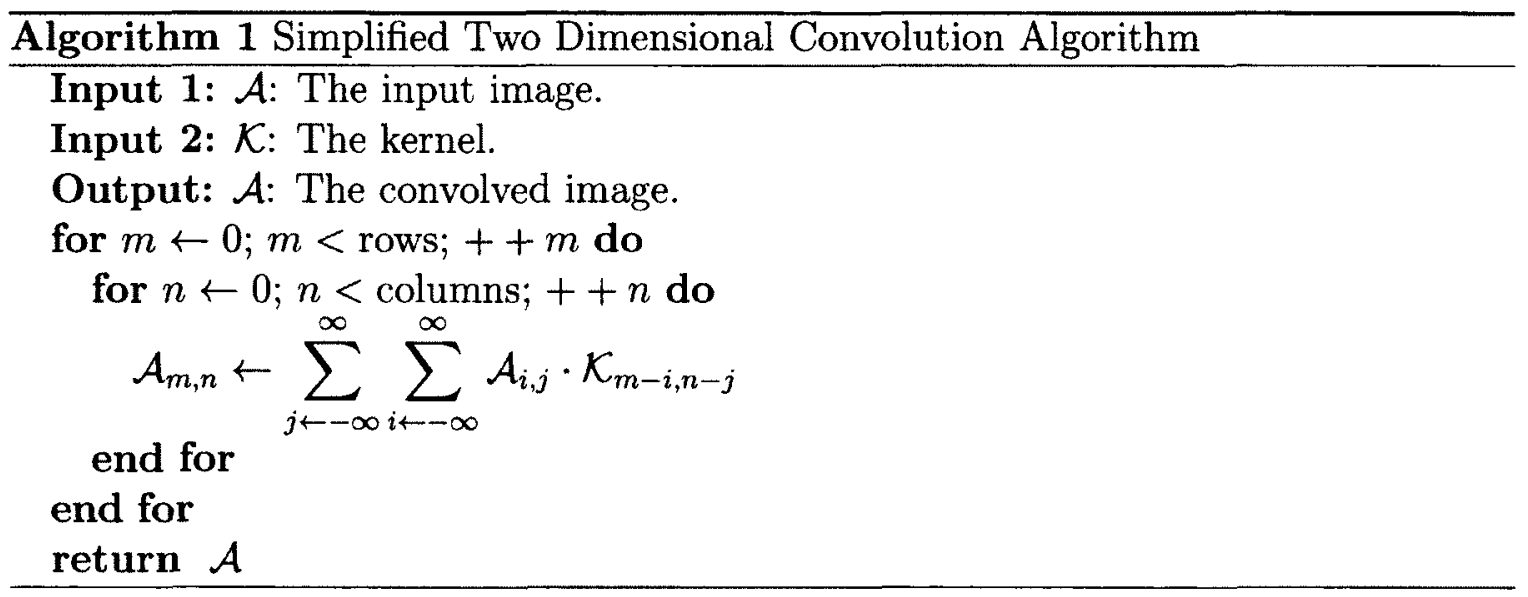

An arbitrary kernel can be chosen for to be used in the convolution algorithm. If no kernel is provided, the default is a $5 \times 5$ Gaussian kernel. Performing the convolution with this kernel is equivalent to applying Gaussian blur to the target image. This operation can either improve or reduce the quality of the image, this is dependent on the state of the input image.

\subsubsection{Pixel-Damage-Based Noise}

Pixel-damage ${ }^{1}$ is a type of noise which is usually added at the raw input stage. If the sensor array portion of the fingerprint scanner is damaged, it is possible for

\footnotetext{
${ }^{1}$ This type of noise is commonly referred to as "salt-and-pepper noise".
} 
certain pixels to be "knocked-out" or inverted. The algorithm developed is intended to simulate this type of noise, where pixels will get "flipped" with a certain probability.

Pixel-damage is performed by using Algorithm 2, where $\mathcal{A}$ is the one dimensional array of the raw fingerprint-image data, $|\mathcal{A}|$ is the size of $\mathcal{A}$, and $p$ is the probability of causing pixel damage to each individual pixel. The pixel damage itself is caused by setting a pixel $\mathcal{A}_{i}$ to $255-\mathcal{A}_{i}$ thus "flipping" its value.

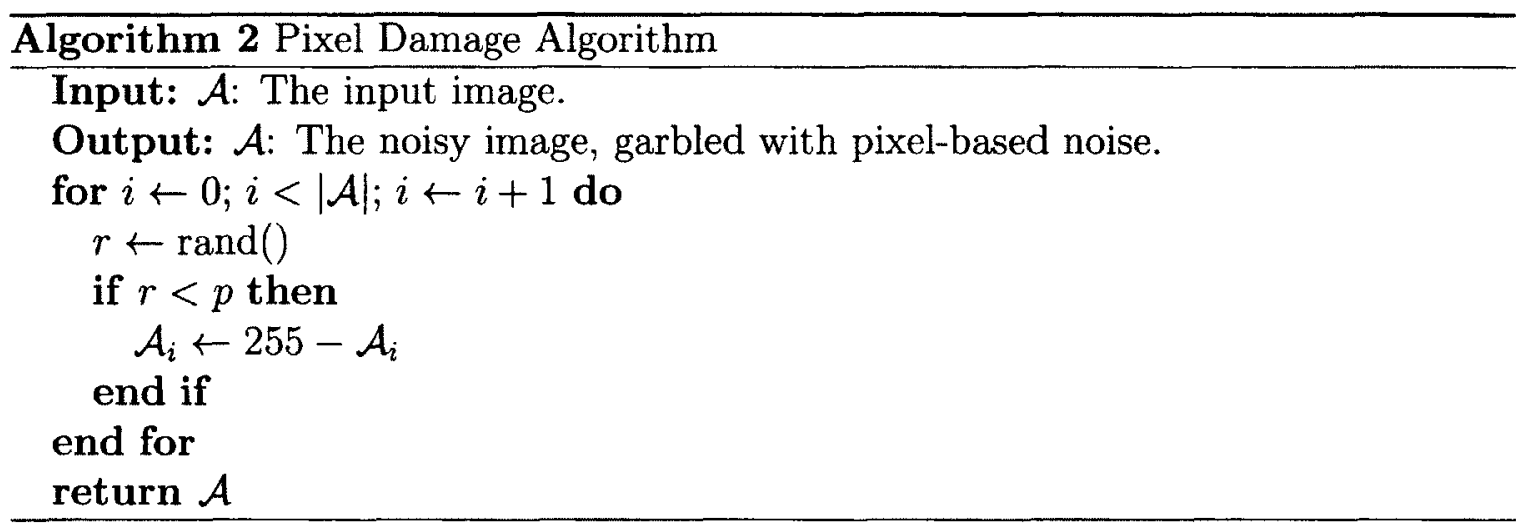

\subsubsection{Additive Gaussian Noise}

In order to simulate additive noise due to sensor devices, another algorithm was included which garbles the target image using Gaussian noise. Additive Gaussian noise simulates a noisy sensor adding "white-noise" to the target image.

Gaussian additive noise was generated by adding a normally-distributed random number to each of the pixels. The random number was generated using a Box Muller Transform (BMT) random number generator (Algorithm 3), where $\sigma$ is the desired standard deviation and $\mu$ is the desired mean. Algorithm 4 describes how the numbers generated by Algorithm 3 are added to the image data, $\mathcal{A}$.

The additive Gaussian noise will cause the gradients between the ridges to become rough and may cause the minutia-extraction algorithm to produce incorrect results. This will, in turn, give the minutia matching algorithm spurious minutiae or prevent it from finding some minutiae. 

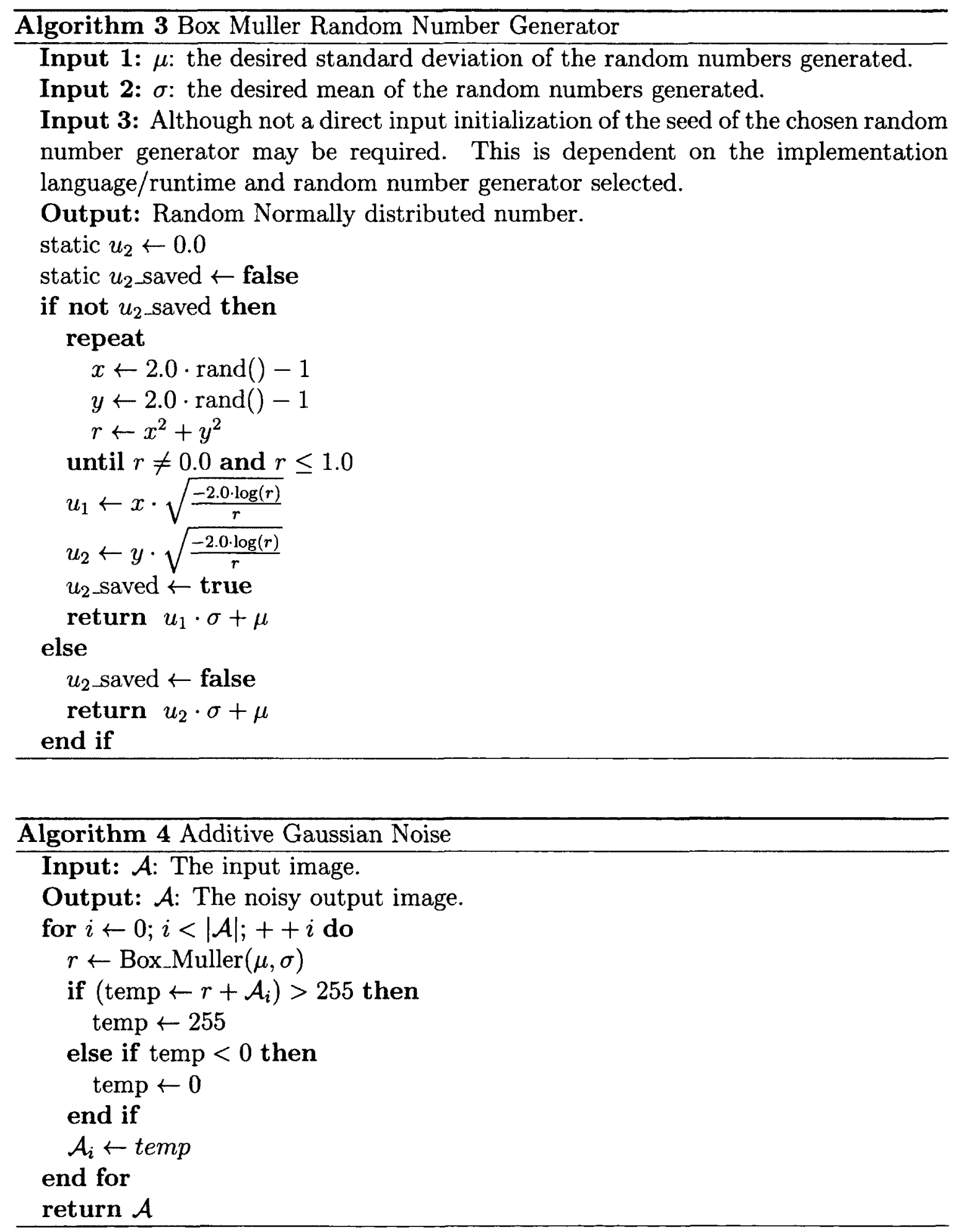


\subsection{Image Enhancement Methods}

As previously mentioned there are a number of different techniques for improving the quality of fingerprint images (with respect to fingerprint recognition). The most common techniques are covered in this section.

\subsubsection{Gaussian Blur}

Gaussian blurring is a common technique used by many different fingerprint matching algorithms since it effectively reduces Gaussian noise (also known as "white noise"), which could have been added by virtue of the tools used to obtain the raw fingerprint data.

Gaussian blur is performed fairly simply by performing a 2-dimensional convolution with a Gaussian kernel given by Eq. (3.1).

$$
G(x, y)=\frac{1}{2 \pi \omega} e^{-\frac{x^{2}+y^{2}}{2 \omega^{2}}} .
$$

This process has the effect of reducing the amount of "white noise" or "Gaussian noise" in the image.

\subsubsection{Contrast Adjustment and Normalization}

There are a number of different techniques for contrast adjustment and intensity normalization. One of the more common techniques used is a pixel-wise normalization using the actual mean and variance, $\mu$ and $\sigma^{2}$, respectively, as well as the target mean and variance, $\mu_{0}$ and $\sigma_{0}^{2}$, respectively. Each input pixel $I(i, j)$ is converted to its corresponding output pixel $G(i, j)$ using Eq. (3.2) [2].

$$
G(i, j)= \begin{cases}\mu_{0}+\sqrt{\frac{\sigma_{0}^{2}(I(i, j)-\mu)^{2}}{\sigma^{2}}} & \text { if } I(i, j)>\mu \\ \mu_{0}-\sqrt{\frac{\sigma_{0}^{2}(I(i, j)-\mu)^{2}}{\sigma^{2}}} & \text { otherwise. }\end{cases}
$$




\subsubsection{Gabor-Filtering-Based Fingerprint Image Enhancement}

A technique presented by Hong, Wan, and Jain [2] for recovering high noise areas on fingerprint images uses an adaptive Gabor filtering scheme to significantly reduce noise and to repair damage like broken ridges and disparity in contrast.

The technique has 5 stages that are similar to those used by other image enhancement methods:

1. Contrast Normalization

2. Local Orientation Estimation

3. Local Frequency Estimation

4. Region Mask Estimation

5. Filtering

Steps 4 and 5 are where this technique deviates from most other fingerprint image enhancement methods. Step 4 creates a region mask by splitting the image into classified regions which are either "recoverable", and which can be reconstructed or filtered of noise, or "unrecoverable" in which case the noise or damage is too great and the region should be ignored. Step 5 uses a bank of Gabor filters tuned to the local ridge orientation and ridge frequency. By using the information provided by the frequency and orientation of parallel ridges, a bandpass filter can be configured to remove the unwanted noise in small local regions, and subsequently the entire fingerprint image.

In order to apply a Gabor filter, given in Eq. (3.3), to the fingerprint image, three parameters are required:

1. The frequency of the ridges in the given region, $f$.

2. The orientation of the ridges in the given region, $\phi$

3. The standard deviations of the Gaussian envelope, $\delta_{x}$ and $\delta_{y}$. 


$$
\begin{aligned}
h(x, y: \phi, f) & =\exp \left\{-\frac{1}{2}\left[\frac{x_{\phi}^{2}}{\delta_{x}^{2}}+\frac{y_{\phi}^{2}}{\delta_{y}^{2}}\right]\right\} \cos \left(2 \pi f x_{\phi}\right) \\
\text { where }: x_{\phi} & =x \cos \phi+y \sin \phi \\
y_{\phi} & =-x \sin \phi+y \cos \phi .
\end{aligned}
$$

The frequency and orientation are obtained based on the current region being filtered. The selection of the values of $\delta_{x}$ and $\delta_{y}$ involves a trade-off. The large the values, the more robust the filter will be to noise; however the more likely is that the filter will create spurious ridges and valleys [2]. Similarly, smaller values of these parameters will create less spurious ridges, but be less effective in removing noise [2]. The enhanced image $E$ is obtained using Eq. (3.6):

$$
E(i, j)= \begin{cases}255 & \text { if } R(i, j)=0 \\ \sum_{u=-w_{g} / 2}^{w_{g} / 2} \sum_{v=-w_{g} / 2}^{w_{g} / 2} h(u, v: O(i, j), F(i, j)) G(i-u, j-v) & \text { otherwise }\end{cases}
$$

where $G$ is the normalized fingerprint image, $O$ is the orientation image, $F$ is the frequency image, $R$ is the recoverable mask obtained from step 4 , and $w_{g}$ is the size of the Gabor filter.

\subsection{Additional Image Processing Tools Used}

In the development of our thesis, we used several tools produced by the National Institute of Standards and Technology (NIST) for image processing. These tools are all part of the the NIST Biometric Image Software (NBIS) toolkit.

A Wavelet/Scalar Quantization (WSQ) compression algorithm, and the corresponding decompression algorithm ("cwsq" and "dwsq" respectively) were used as part of the fingerprint identification system.

In order to convert images between different formats which are more widespread (formats including JPEG and PNG), the Python Image Library (PIL) was used. 
These image manipulation tools were utilized to draw minutiae on fingerprint images, and also to convert raw and WSQ format images into PNG for use with existing image viewers.

\subsection{Chapter Summary}

In this chapter we have covered one of the most fundamental portions of the fingerprint matching process, image processing. Specifically we have discussed the noise inherent in the fingerprint matching problem, several methods of garbling fingerprint images, several commonly used fingerprint image enhancement methods, and the image processing tools used throughout the study. 


\section{Chapter 4}

\section{File Format Considerations}

Fingerprint image libraries exist which, without proper measures, would create impractical storage requirements. The FBI's fingerprint database is upwards of 66 million templates, many with full ten-print cards ${ }^{1}$ and even multiple sets of prints [8]. The following chapter describes the measures which have been taken to solve this particular problem: the creation of the WSQ image format for fingerprint images in identification systems. In addition, this chapter will touch on the different file formats which are used in the developed system.

\subsection{Importance of Compression}

Fingerprint data is most often stored as images. This not only preserves as much information about the fingerprint as possible, it also permits the use of new feature extraction and fingerprint matching techniques with legacy fingerprints, potentially producing new and interesting results.

The disadvantage of storing fingerprints as images (instead of storing files as lists of extracted features) is that images are quite large in comparison to the feature information alone. Indeed, much of the fingerprint image is noise when it concerns fingerprint matching algorithms [2]. For small databases of fingerprints this is not an issue. But for large organizations like the FBI, the cost of maintaining loss-less

\footnotetext{
${ }^{1}$ For example ten-prints, which would include each of the subject's 10 fingerprints.
} 
images is too prohibitive when the size of their database is taken into consideration (upwards of 66 million templates) [8].

In order to solve this storage problem, it is obviously desirable to compress the fingerprint image data as much as possible while still retaining all the useful feature information.

\subsection{Problems with Pre-Existing Image Compres- sion Techniques}

A number of techniques for image compression are in use today. Both loss-less and lossy compression techniques for the commonly used PNG, TIFF and JPEG formats exist. While many of these techniques provide adequate compression ratios for fingerprint images, it is often the case that a substantial portion of the useful information contained within these fingerprints is lost [4].

Loss-less compression techniques provide a compression factor of approximately 2 on gray-scale images which was considered inadequate by the FBI [1]. Other popular compression techniques, when tuned to the FBI's compression requirements of 0.75 bits per pixel, remove too much information and produced interfering "block-effects" which are visible in Figure 4.1 [1]. The effect of this type of distortion on minutia collection is obviously detrimental. The "block" noise can "shear" ridges which, in combination with the ridge thinning process, may cause spurious ridge-ending type minutiae. The extent to which spurious minutiae are produced is dependent on the severity of the "block" noise and the method used to thin the fingerprint ridges [1].

\subsection{Wavelet Scalar Quantization Compression}

In response to the lack of a suitable compression algorithm, particularly when the lack of storage became a crucial issue for the FBI, research into alternatives became necessary. The compression standard produced as a product of the research from Hopper, Preston, Bradley and Brislawn, and the FBI, is known as the Wavelet/Scalar 
Quantization (WSQ) compression scheme [1, 32, 33].

WSQ compression is based on an adaptive scalar quantization approach [33]. It achieves the compression level required by the FBI standards and causes only small loss in feature data.

The following steps summarize the WSQ compression algorithm:

1. A 2-dimensional discrete wavelet transform is applied to the fingerprint in order to decompose it into 64 different spatial frequency bands.

2. The output of the transform is quantized into discrete values, which is one phase of the actual compression portion of the algorithm. This is also the step that cannot truly be reversed and leads to the data loss.

3. In order to produce the final compressed image, Huffman-coding is used on the output of the quantization step.

WSQ compression is an excellent technique for fingerprint image compression. It provides a compression ratio of up to $15: 1$ with an associated acceptable data loss $[1,32,33]$.

Figure 4.1 exemplifies the "block-effects" on the ridges which result from other lossy compression techniques. In the JPEG compressed image, not only are the ridges no longer smooth, but features like pores have been completely eliminated from the JPEG compressed image. The "block-effects" that are present on the ridges create issues for minutia detection as a result of the harsh gradient changes from "block-to-block". Additional details like small outcroppings on the ridges are often eliminated or rendered indistinguishable as a result of block-noise. In contrast, the WSQ compressed image does not have any "block-effects" and its ridges are much smoother. Pores are visible but still may be more difficult to extract from the WSQ compressed image [1]. Each of the features which have been degraded or eliminated by the JPEG compression technique are all applicable in court [1]. 

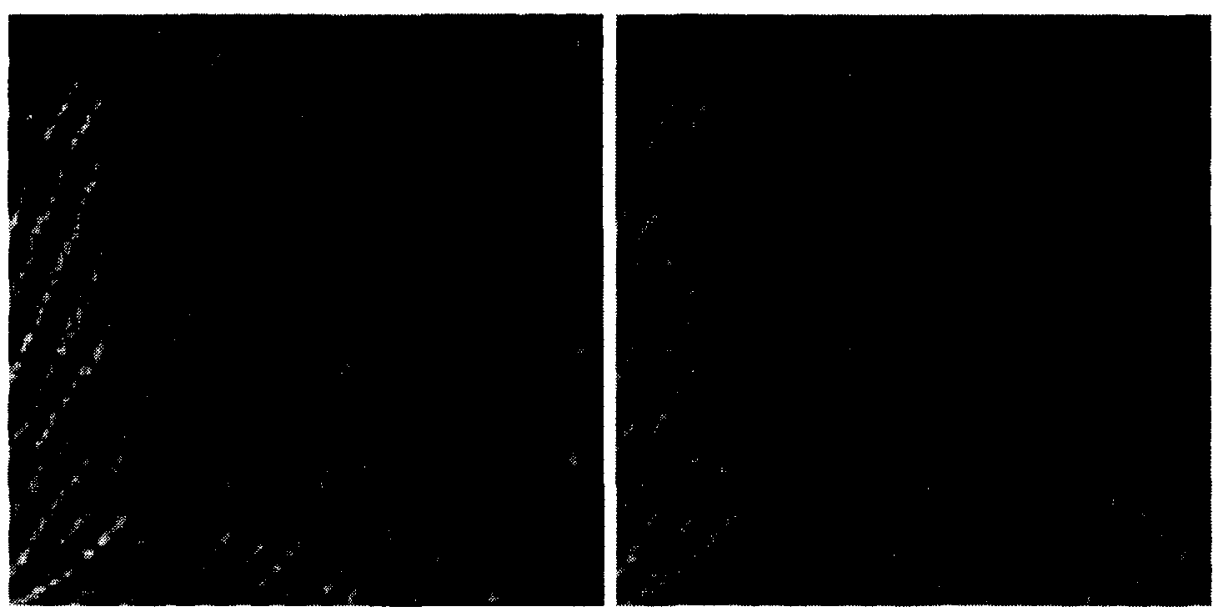

Figure 4.1: Examples showing the effects of two different image compression types. In the left panel is a fingerprint image compressed using standard JPEG compression and in the right panel the same image compressed using the FBI's standard WSQ compression. The compression algorithms were tuned to the same compression ratio. The magnified portion of the left image shows the blocks produced by the JPEG compression algorithm. (Note: The effect may not be as pronounced in some PDFreaders due to image processing performed by these applications.) 


\subsection{File Formats Used in The Developed System}

In addition to the WSQ image format, several other file-formats which are not commonly used in fields other than fingerprint recognition are used in the system. These formats include the XYT and MIN file formats, both of which are of the plain-text file-type.

The XYT file-format stores minutiae. Each line of an XYT file represents a minutia, with space delimited Cartesian coordinates, $x$ and $y$, as well as the angle of that minutia's most closely associated ridge, $\theta$, and finally the "quality" of that minutia, $u$. This file format is used by many fingerprint recognition algorithms [25] and is the primary output of the "mindtct" program (this program is discussed in more detail in Chapter 7).

The MIN file-format also stores minutiae, however it stores more features and detail on each minutia than the XYT file-format. Each line represents a minutia, as with the XYT files. Instead of being space delimited MIN entries are colon delimited. The data stored for each minutia includes:

- A minutia "id" number.

- The $x$ and $y$ Cartesian coordinates.

- A discretized ridge angle value.

- A "reliability" measure.

- The minutia's type (ridge ending or bifurcation).

- The Cartesian coordinates of the nearest neighboring minutia.

- The ridge count between the represented minutia and its nearest neighbor.

The MIN format is used when more details about the minutiae are required by the matching algorithm, each representation of the fingerprint's minutiae are available to algorithms which are added to the developed system. 


\subsection{Chapter Summary}

In this chapter we have discussed the file format considerations in the construction of a fingerprint matching system. Although file formats are a minor topic, in this thesis, it is still important to understand the most common fingerprint image and minutia file formats as well as the challenges involved in storing them. This will permit the integration of biometric tools and algorithms. 


\section{Chapter 5}

\section{Pattern Recognition Methods}

In the earlier chapters of this thesis, the components required to create a fingerprint identification/verification system were described in detail, and various techniques for accomplishing each step of the pertinent recognition phases were formulated. The focus of the rest of this thesis is the matching phase of the fingerprint identification process. This chapter covers the fingerprint matching algorithms that have been implemented and used in the fingerprint identification system that we have developed. These algorithms were chosen from the wide selection of methods reported in the literature, and were implemented or procured through the use of open-source software.

The two main categories of minutiae matching algorithms were discussed in Section 2.6.4. In this regard, a brief survey of global and local techniques is provided in Sections 5.1 and 5.2 respectively.

In this chapter, we will describe the operation of each of the algorithms which have been implemented from previous works and subsequently integrated into the fingerprint recognition system that we have developed.

\subsection{Survey of Global Matching Techniques}

The following is a brief survey of well-known global matching techniques. The intention of this section is to provide some background into the operation of these acclaimed global matching algorithms. 


\subsubsection{Hough Transform}

One of the most common global techniques are the group of Hough transform-based matching algorithms. These techniques are often based on the Generalized Hough Transform (GHT) analogous to the one reported in [34] (and mentioned in [1]), which is used to adapt the fingerprint matching problem "onto" a simple noise-tolerant point-to-point matching problem. An example usage of this technique can be found in [24], and is also described in detail in Section 5.3.2.

\subsubsection{Ridge Feature Alignment Approach}

Another global technique reported by Jain et al. [30] uses a ridge-based feature alignment approach, transforming the minutiae matching problem into a string matching problem. The algorithm proceeds with the following steps:

1. Align the minutiae-based on their ridge features.

2. Convert the minutiae from each template into symbolic string representations: $P_{p}$ and $Q_{q}$.

3. Match the two strings using a dynamic programming algorithm.

4. Score the fingerprint based on the minimum edit distance between the two strings.

The alignment, in turn, proceeds by using a property of a point-pattern and an associated curve (in the case of a fingerprint, a ridge) to achieve an estimation of the transformation parameters which align the two fingerprints [30]. The details of how this achieved is omitted here to avoid confusion.

Conversion of the, now aligned, minutiae into strings is performed by sorting them by using the increasing order of the angle of the most closely associated ridge. These minutiae and their features are concatenated in this order to form symbolic strings $P_{p}$ and $Q_{q}$.

An interesting feature of the matching process is that it uses adaptive bounding boxes to tolerate distortion. The amount of distortion that it tolerates increases, as 
necessary, at a given learning rate. This results in a slightly mobile bounding box which can shift its location in order to accommodate local distortion due to skin plasticity [30].

\subsection{Survey of Local Matching Techniques}

The following is a brief survey of some of the more well-known local-scheme matching techniques. As with the section of global matching techniques, this section is intended to provide some background in the area of local matching techniques.

\subsubsection{Feature-Vector Based Matching}

One of the oldest local-scheme algorithms for minutiae matching creates a feature vector in order to represent each minutia, and was reported by Hrechak and Mchugh [35]. This feature vector consists of information relating not only to the minutia that the vector was created to represent, $m_{i}$, but also that minutia's neighbors. The vector holds a series of numbers representing the quantity of each type of minutia which is within the "neighborhood" of $m_{i}$. The neighborhood is constructed by collecting the minutiae which are within a radius $r$ of $m_{i}$. The feature vector is of the form $v_{i}=\left[v_{i, 1}, v_{i, 2}, \ldots, v_{i, 8}\right]$ where $v_{i, j}$ represents the number of minutia of type $j$ found within the radius $r$ of minutia $m_{i}$ [35]. Eight different types of minutia were considered, but we maintain that the type of minutia is considered an unstable feature, as this feature (i.e., the type of a specific minutia) can be difficult to determine in an automated fashion, and can be incorrectly recorded at even the image level as even slight variance in pressure when the print is taken can change the interpreted type of a minutia [1].

As mentioned in [1] and reported in [36], this feature vector technique can be improved upon in several important ways. In addition to storing information about the type of the minutiae in the local neighborhood of the central minutia $m_{i}$; one can store information about the distance from $m_{i}$ of each minutiae, the ridge count between each minutiae and $m_{i}$, the direction in which the minutiae are relative to 
$m_{i}$, and the total number of nearby minutiae. This helps to counteract the instability of the minutiae-type-feature, as well as compromise for different types of distortion than accounted for by recording only the minutia type.

\subsubsection{Adjacency Graph Matching}

Another local technique reported by Ratha et al. [6] uses a "star-representation" of minutiae, their local region and their structural relationships, to create a minutiae adjacency graph. Once created, this adjacency graph would be used to perform matching using weighted comparison techniques. In addition the algorithm uses two phases for matching, a "strict matching" phase and an "extension phase".

The strict matching phase performs a weighted matching between minutiae pairs with tolerances between each of their attributes. Angles of the edges from each star are used, instead of the angles of the most closely associated ridge, in order to allow the star minutia representation to be rotationally independent.

The "extension phase" involves improving the certainty of matches by accumulating more evidence. Matches obtained through the strict phase are extended using matching techniques with relaxed thresholds.

This two phase matching system was designed primarily to mimic the way a fingerprint expert might examine a fingerprint. By first selecting a minimum set of minutiae (from both fingerprints) which can be reliably matched, he would employ a basis for expanding the amount of evidence which corresponds between the two fingerprints.

An improvement of this technique is reported in [25]. This improvement is known as the "K-plet" representation, and is discussed in detail in Section 5.3.3.

\subsubsection{Geometric Clustering-Based Matching}

This matching technique is based on using a fuzzy bipartite graph to match the two fingerprints and was reported by Fan et al. [37]. The query fingerprint's minutiae constitutes one of the independent sides of the bipartite graph, and the database fingerprint's minutiae represents the other independent side of the bipartite graph. 
The minutiae are clustered using the maximin algorithm reported in [38] into bounded boxes. Important attributes of each of these clusters are then themselves measured.

These characteristics are used to distinguish between different clusters and include the following: $N O B$; which is the number of bifurcations in $c_{i}, N O E$; which is the number of endpoints in $c_{i}, W H$; which is the ratio of the width over the height of the rectangular bounding box of $c_{i}, O T$; which is the orientation angle of all ridge pixels in $c_{i}$, and $S_{k}$; which are 20 different measurements based on the results from different $5 \times 5$ masks [37].

Each of these attributes, along with the minutiae clusters, are converted into representations of fuzzy sets, each possessing their own membership function to determine a "degree of belonging". The optimal solution to the matching of the two fingerprints using this technique is the minimum cost between the corresponding nodes in the graph [37].

\subsection{Implemented Recognition Methods}

Several techniques were implemented in the system which we have developed. These include a technique based on the GHT, one based on matching local graphs, and the bozorth3 algorithm ${ }^{1}$. These methods were either implemented from fundamentals or obtained from open-source software so as to be used with the system. This section describes some of the major decisions made during the development and integration process, as well as the operating details of the implemented/included matching techniques.

\subsubsection{Development Notes}

The programming language chosen for the development of the algorithms was $\mathrm{C}++$. It was chosen because it is widely used, and produces very fast binaries, and it should be beneficial in the event that another party should wish to use the implementation

\footnotetext{
${ }^{1}$ Also referred to as Bozorth's algorithm.
} 
at a later time. The drawback of the language choice is that $\mathrm{C}++$ is a poor choice for prototyping due to its development cycle. As a result, for the purposes of prototyping, we used the language Common Lisp. Common Lisp's highly dynamic runtime permitted rapid development and re-factoring on a "live" system. Once the prototypes reached a level which indicated that the technique was implemented properly and functioned as indicated in the relevant literature, they were translated to $\mathrm{C}++$.

We also developed a complete library for parsing XYT and MIN files using the clppcre library, so as to accomplish the data extraction with regexps. This XYT/MIN parser was later translated to $\mathrm{C}++$ using the widely used Boost library collection for regexp support.

\subsubsection{Global-Scheme: Hough Transform Alignment-based Match- ing}

Hough Transform techniques are often used in image processing and pattern matching algorithms. The Hough Transform is essentially a feature extraction technique, frequently used for tasks such as line and edge detection. In a pattern matching algorithm, such as fingerprint matching, one can use the GHT to perform elastic point pattern matching as described in [34] and used in [24].

\section{Generalized Hough Transform}

The regular Hough Transform can only be used to detect objects that can be described with an equation ${ }^{2}$, whereas the GHT can be used to detect arbitrary objects described with a template and with a set of appropriate transformations.

The GHT, as applied to the minutia matching problem, is simply a mapping of one minutiae set to another [24]. The goal is to find an appropriate transformation from the query template's minutiae to each database fingerprint template. Consider two minutiae sets [24]:

$$
\mathcal{P}=\left\{\left(p_{x}^{1}, p_{y}^{1}, p_{\theta}^{1}\right), \ldots,\left(p_{x}^{P}, p_{y}^{P}, p_{\theta}^{P}\right)\right\}
$$

\footnotetext{
${ }^{2}$ Hough Transforms have been used to detect lines, circles and other similar objects.
} 


$$
\mathcal{Q}=\left\{\left(q_{x}^{1}, q_{y}^{1}, q_{\theta}^{1}\right), \ldots,\left(q_{x}^{Q}, q_{y}^{Q}, q_{\theta}^{Q}\right)\right\}
$$

where $\mathcal{P}$ is one of the database template's fingerprint sets, and $\mathcal{Q}$ is the query fingerprint template's minutiae set. In order to match these two sets of points together, the space of all possible transformations (sometimes referred to as the Hough Space) of the point-set are defined by the following transformation parameters: scale $(s)$, rotation $(\phi)$, and Cartesian displacement $(\Delta x$, and $\Delta y)$. The Hough Space is obviously infinitely large and must be restricted in order to deal with the complexity of exploring it. The Hough Space is restricted based on knowledge of the problem domain. For example, it has been empirically determined that rotational noise of fingerprints in a fingerprint database does not often exceed $\pm 20 \%$ [39]. With restrictions like this in place, the range of accepted transformation parameters is discretized. The transformations themselves are all of the form shown in Eq. (5.1) [24]:

$$
F_{s, \phi, \Delta x, \Delta y}\left(\begin{array}{c}
x \\
y
\end{array}\right)=s\left(\begin{array}{rr}
\cos \phi & \sin \phi \\
-\sin \phi & \cos \phi
\end{array}\right)\left(\begin{array}{c}
x \\
y
\end{array}\right)+\left(\begin{array}{c}
\Delta x \\
\Delta y
\end{array}\right) \text {. }
$$

In order to determine the best parameters to give the transformation from $\mathcal{P}$ into $\mathcal{Q}$ we used Algorithm 5, which was reported in [24]. The algorithm iterates through all possible pairings of points between $\mathcal{P}$ and $\mathcal{Q}$ and subsequently uses each of the discretized values of $\phi \in \Phi$ which will bring $p_{\theta}$ to within tolerance of $q_{\theta}$. Once it finds these values of $\phi$ it iterates through each of the possible values of $s$ and solves for $\Delta x$ and $\Delta y$ using Eq. (5.1). Once each of the values of $s, \phi, \Delta x$, and $\Delta y$ have been determined, a vote is cast into the bin representing these transformation parameters within the Hough Space $^{3}, \mathcal{A}$ [24].

Once each combination of minutia pairs from $\mathcal{P}$ and $\mathcal{Q}$ have been attempted, the transformation with the most votes in its bin is chosen. Subsequently, the minutia matching algorithm becomes a simple point-matching problem [34].

An example of the alignment transformation produced by Algorithm 5 between two fingerprints from the FVC2002 database is shown in Figure 5.1.

\footnotetext{
${ }^{3}$ As well, votes are cast into nearby bins in order to reduce the algorithms sensitivity to noise and improve its overall results.
} 

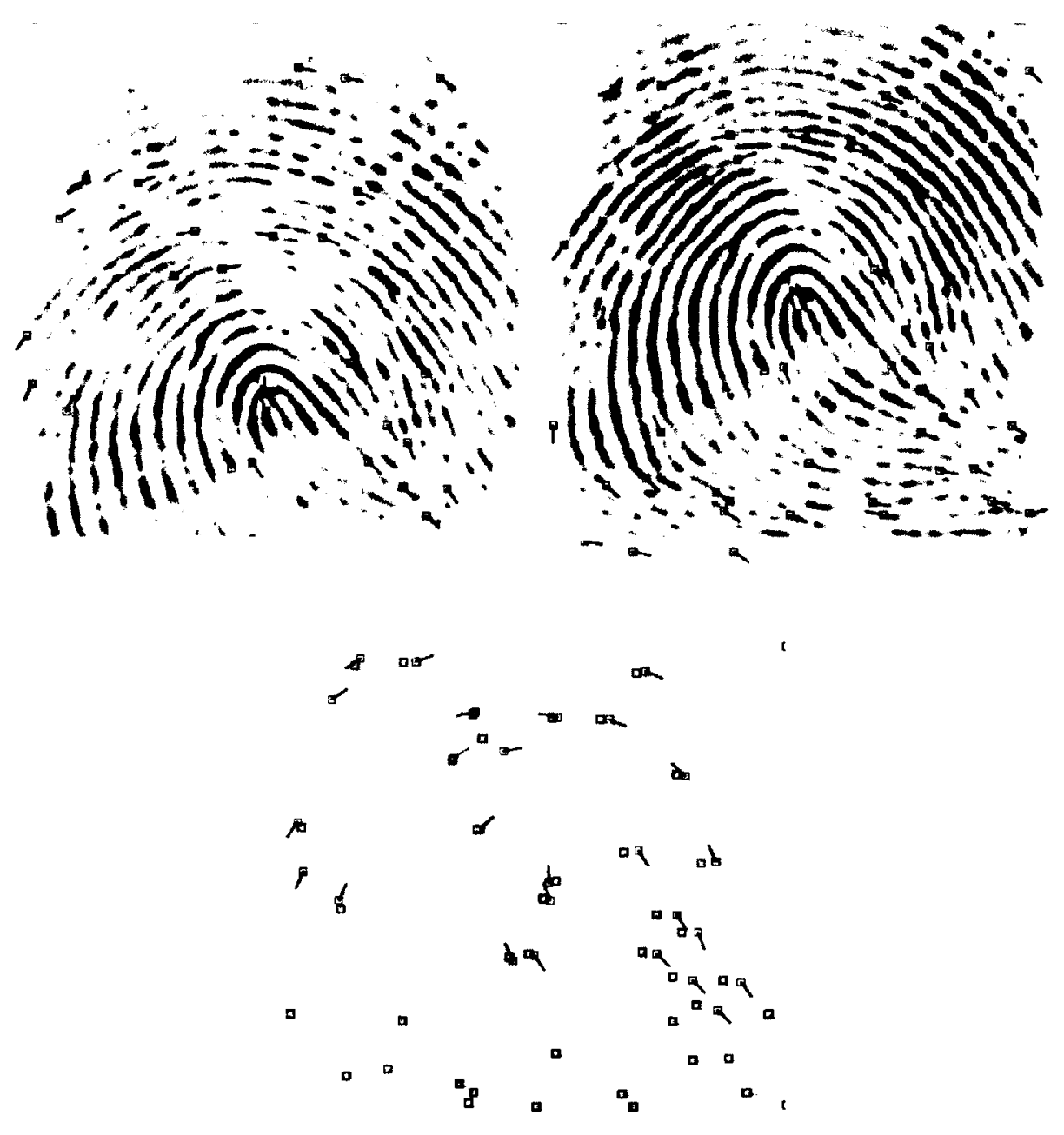

Figure 5.1: Diagram showing the alignment produced by Algorithm 5. The top two images depict the two fingerprints, from the same digit, with their detected minutiae, and the bottom image is the resulting alignment between the two sets of minutiae. One set of the minutiae is coloured green with its $\theta$ indicator coloured orange, while the other set of minutiae is coloured red with its $\theta$ indicator coloured blue. 


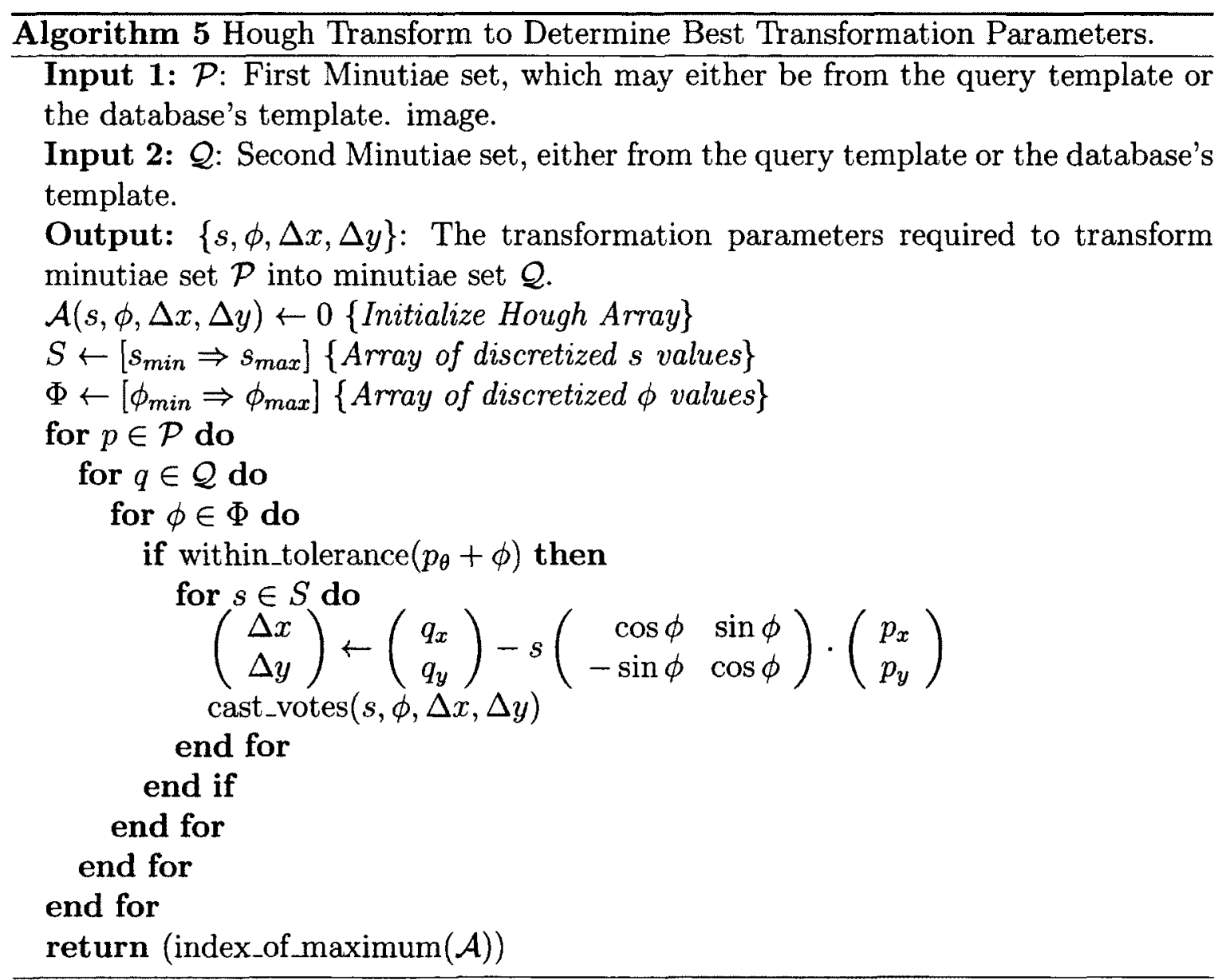




\section{The Point-Matching Problem}

Once transformed, all that must be done to fully match one fingerprint against the other is to do an $N \times M$ comparison of the query template fingerprint's minutiae against the database fingerprint's minutiae. Two minutia points are considered a match if they satisfy the two requirements in Eq. (5.2) and Eq. (5.3) where $\tau$ is the similarity threshold between the angles of two different minutiae and $\delta$ is the threshold on the Euclidean distance between two different minutiae.

$$
\begin{aligned}
& \tau>\min \left(\left|p_{\theta}+q_{\theta}\right|, 360-\left|p_{\theta}-q_{\theta}\right|\right) \\
& \delta>\sqrt{\left(q_{x}-p_{x}\right)^{2}+\left(q_{y}-p_{y}\right)^{2}} .
\end{aligned}
$$

The implemented point-matching algorithm uses a pseudo best-match algorithm. The algorithm pairs each minutia with its "best-match" from the remaining set, and then removes that match from the minutiae checked by subsequent point matches. This will obviously not obtain the best matching pairs but will approximate the best. This algorithm is summarized in Algorithm 6.

Once all the minutiae have been paired with their approximate "best" matches, the match score is computed. The method of computing the match score is listed in Eq. (5.4). This is a common method of computing the match score between two particular fingerprints. The paired fingerprints which score above a certain threshold are considered to be matching.

$$
\text { score }=\frac{\mid \text { results }\left.\right|^{2}}{|\mathcal{P}| \cdot|\mathcal{Q}|},
$$

where "results" is a vector containing the matched minutia pairs".

\section{Development Notes}

When the algorithm was originally implemented it was found to take an average of 30 seconds, on the available machines, to compute the match score between two

\footnotetext{
${ }^{4}$ We use the absolute value operators "II" indicate the size of a set when they are applied as such. Otherwise when applied to a normal value it is to indicate the "absolute value" of that value.
} 


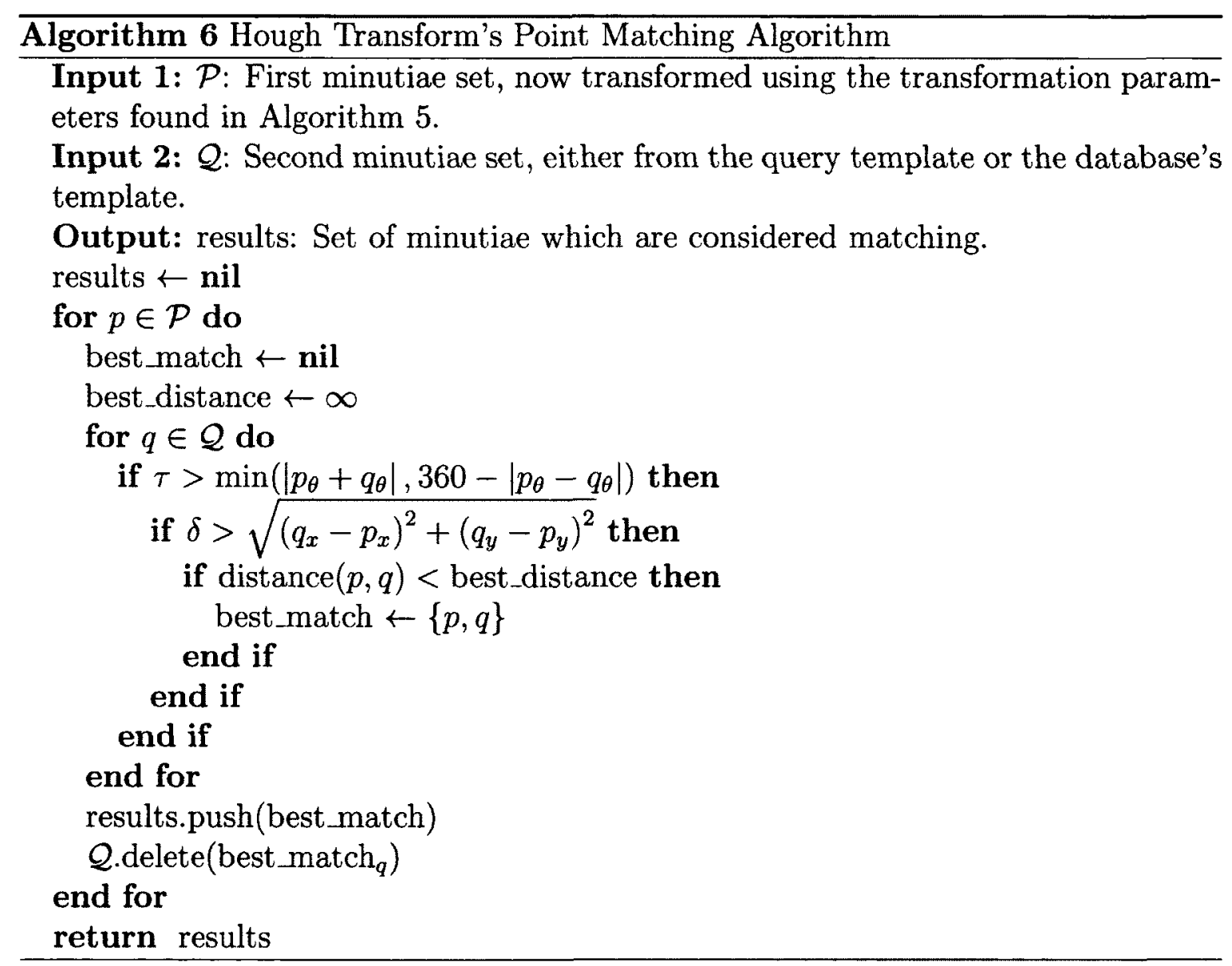


fingerprints. This execution time is approximately two orders of magnitude larger than that of the execution time of the bozorth3 algorithm from the NBIS package.

In an attempt to improve the speed of the Hough transform-based algorithm, the parameters of the algorithm were adjusted. Observe that the adjustable parameters of the Hough algorithm include the following:

- The maximum size, minimum size and resolution of the discretized scaling factors $s_{\min }, s_{\max }$ and $s_{n}$ respectively.

- The maximum and minimum rotation of the angle of the most-closely associated ridge, $\phi_{\min }$ and $\phi_{\max }$.

- The maximum and minimum horizontal displacements $x_{\min }$ and $x_{\max }$.

- The maximum and minimum vertical displacements $y_{\min }$ and $y_{\max }$.

These parameters define the size of the Hough array and are the constants affecting the number of iterations required by Algorithm 5 . The initial parameters were generous and included scaling up to $\pm 30 \%\left(s_{\min }=0.7, s_{\max }=1.3\right.$ and $\left.s_{n}=0.1\right)$, rotation up to $360^{\circ}$ in both directions $\left(\phi_{\min }=-360\right.$, and $\phi_{\max }=360$ ), and displacement up to 300 pixels $\left(x_{\min }=y_{\min }=-300, x_{\max }=y_{\max }=300\right)$.

The parameters which affect the number of iterations required by Algorithm 5 include $s_{\min }, s_{\max }, s_{n}, \phi_{\min }$ and $\phi_{\max }$. Fingerprint images vary in orientation by approximately $\pm 20^{\circ}[8,40]$, which gives $\phi_{\min }=-20$ and $\phi_{\max }=20$. The rest of the parameters were adjusted by observing the transformation parameters chosen by the algorithm itself. The maximum parameters chosen for a matching pair of fingerprints was used to choose appropriate values, giving: $s_{\min }=0.98, s_{\max }=1.02, s_{n}=5$, $x_{\min }=-100, x_{\max }=100, y_{\min }=-100$ and $y_{\max }=100$. By using these modified parameters, the execution time of the algorithm was brought to be within milliseconds of the speed of the bozorth3 algorithm.

During tuning we noted a distinct drop off point where the hough transform ceased to produce suitable point alignments for matching. It was difficult to determine an exact level of noise which produced this behavior but the phenomenon was noted several times throughout the tuning process. 


\subsubsection{Local-Scheme: K-plet Representation Matching}

The K-plet representation-based matching algorithm is similar to other local-scheme minutia matching algorithms. As with other local-schemes, the K-plet algorithm creates structures from localized minutiae and performs comparisons on the collection of localized structures of the two fingerprints.

\section{K-plet Representation}

The K-plet representation is at the core of the algorithm. The representation is like that of a similar algorithm which uses "star-like" graphs as local structures for comparing fingerprint minutiae by Ratha et al. [6]. The K-plet representation uses a central minutia $m_{i}$ and $K$ neighbors $\left\{m_{1}, m_{2}, \ldots, m_{K}\right\}$ to create a graph, referred to as $G$. The minutiae are represented as three-tuples $\left\{\phi_{i j}, \theta_{i j}, r_{i j},\right\}$ where $\phi_{i j}$ represents the angle of the edge connecting $m_{i}$ and $m_{j}$ relative to the horizontal, $\theta_{i j}$ represents the relative orientation of minutia $m_{j}$ with respect to the central minutia $m_{i}$, and $r_{i j}$ is the Euclidean distance between minutia $m_{i}$ and minutia $m_{j}$.

Two methods are given by Ratha et al. [25] for the construction of the local K-plet structures.

- The first method is based on determining the $K$ neighbors in terms of their Euclidean distances. This method was found to work fairly well and is simple to implement [25]. The drawbacks of this method is that it does not spread the minutiae contained within the K-plet structure across the fingerprint, reducing the discriminating power of the structure [25].

- The second method was created to provide a "higher-connectivity" between disparate portions of the fingerprint. $K$ neighboring minutiae are selected such that the nearest neighbor is picked from separate quadrants instead of being chosen purely based on proximity [25].

Once a K-plet structure has been constructed for each of the minutia in the fingerprint, their local structural relationships are composed into a directed graph $G(V, E)$. Each vertex $v$ represents a minutia and each edge $(u, v)$ represents the neighboring 
minutia as defined by that minutia's K-plet structure. Each vertex $u$ is colored with attributes $\left(x_{u}, y_{u}, \theta_{u}, t_{u}\right)$, where $x_{u}$ and $y_{u}$ are the Cartesian coordinates, $\theta_{u}$ is the orientation of the minutia, and $t_{u}$ is the minutia type [25].

\section{Graph Matching Algorithm}

Once the graphs have been constructed for both of the fingerprints in question, the actual task of matching the two graphs can be accomplished. Matching proceeds through the use of a dynamic programming technique based on the string alignment problem. Instead of matching the local K-plet structures (which are now represented by nodes and their outward facing edges in the graph) separately, the structures (or nodes of the graph) are matched simultaneously. This is important as it has been determined that matching structures in greedy fashion is sub-optimal $[6,25]$.

In order to match the K-plets in a stable manner, the neighbors of the K-plet's central minutia are sorted in increasing order of the magnitude of their Euclidean distances from the central minutia. This transforms the graph matching problem to that of matching two ordered sequences (as in the string alignment problem) [25].

The groups of K-plets can now be represented as two ordered sequences $S$ and $T$. To solve the alignment problem, the original sequences must be transformed into two sequences $S^{\prime}$ and $T^{\prime}$ which have the following properties [25]:

1. $S^{\prime}$ is formed from $S$ by adding spaces as necessary.

2. $T^{\prime}$ is formed from $T$ by adding spaces as necessary.

3. $\left|S^{\prime}\right|=\left|T^{\prime}\right|$.

The dynamic programming-based approach to solving this problem is as follows: Let the cost of aligning sub-strings $S(1 \ldots i)$ and $T(1 \ldots j)$ be given by Eq. (5.5). The cost of aligning the entirety of both strings $S$ and $T$ can therefore be given by $D[M, N][25]$.

$$
D[i, j]: i \in\{0,1 \ldots M\}, j \in\{0,1 \ldots N\}
$$


The recurrence relation between $D[i, j]$ and the already computed values is used to reduce the run-time, where $D[k, l]$ is optimal $\forall k<i, l<j$. Once all previous sub-problems have been solved, $s_{i}$ and $t_{j}$ can be matched in a number of ways [25]:

1. A cost of $\sigma(s[i], t[j])$ can be introduced between the elements $s[i]$ and $t[j]$.

2. A gap in the string $T$ can be inserted with cost $\sigma(s[i],-)$.

3. A gap in the string $S$ can be inserted with cost $\sigma(-, t[j])$.

\section{Consolidation Approach}

Another important part of the K-plet algorithm is its associated consolidation algorithm, Coupled Breadth First Search (CBFS). The algorithm is based on a breadth first search philosophy as its name suggests, except for two factors [25]. First, traversal occurs in two graphs $G$ and $H$, which have been constructed from the K-plets, simultaneously. Second, the standard breadth first search will visit each vertex in the adjacency list, whereas the CBFS algorithm only visits vertices $v_{G}$ and $v_{H}$ from $G$ and $H$ where $v_{G}$ and $v_{H}$ have been successfully matched.

The CBFS is shown in Algorithm 7, where $V_{G}$ and $V_{H}$ are the sets of vertices in graphs $G$ and $H$ respectively, match_neighbors() attempts to matches neighbors and returns the list of those matched neighbors, and color $(v)$ accesses the color of vertex $v$.

Since the CBFS algorithm requires two starting nodes $i$ and $j$, and the optimal starting nodes are not known, the CBFS is run with all possible starting nodes in the graphs. The pair of starting positions which yield the greatest number of matches are stored and used to produce a matching score. Once the CBFS algorithm has completed, the returned matched minutiae vector, $M$, is used to compute the matching score [25]. The size of the matched minutiae vector, $|M|$, is used in Eq. (5.6) in a similar manner to that used in the Hough-transform-based algorithm in Section 5.3.2 in Eq. (5.4).

$$
\text { score }=\frac{|M|^{2}}{|\mathcal{P}| \cdot|\mathcal{Q}|}
$$




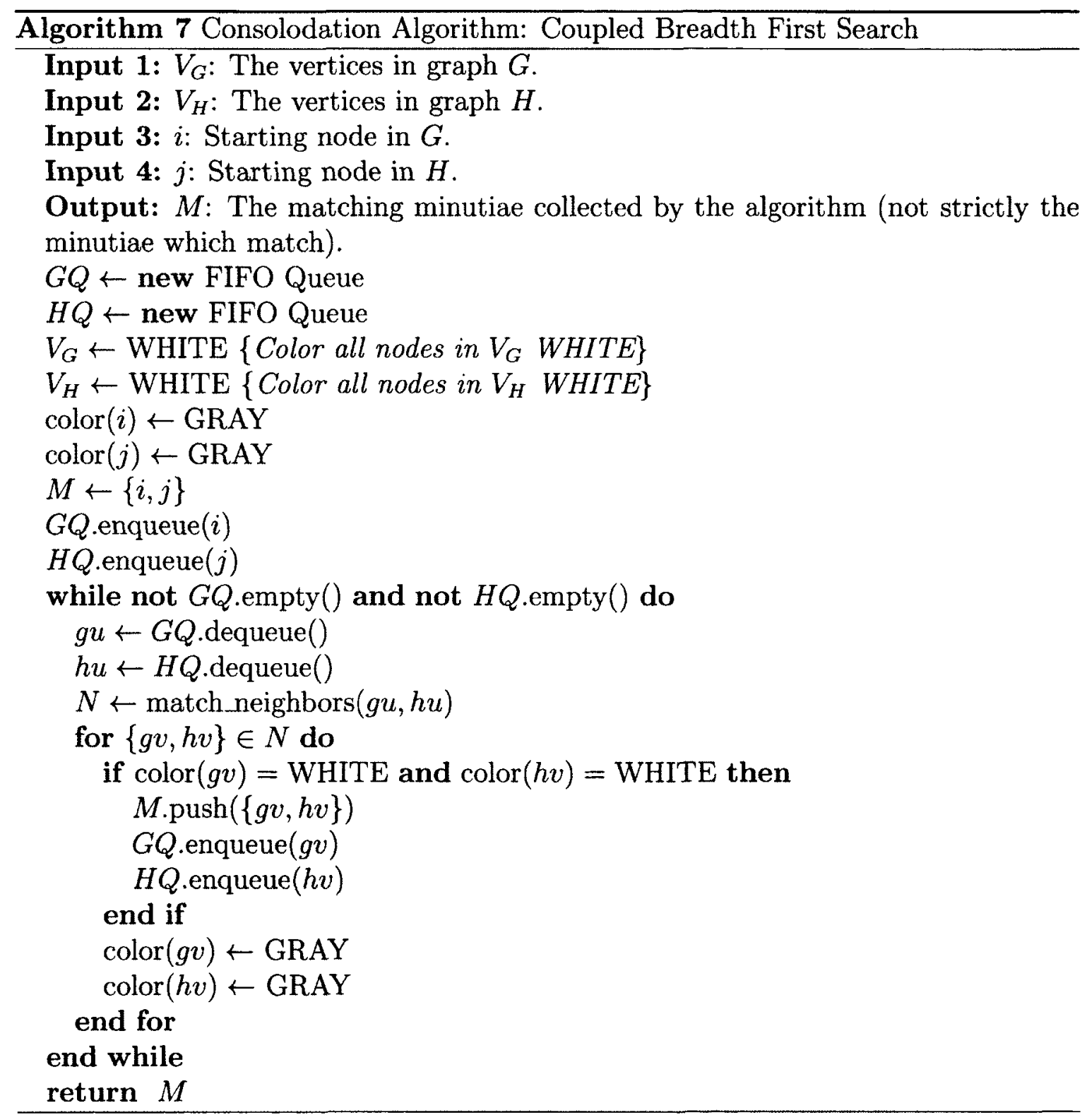




\section{Development Notes}

Much of the included implementation of the K-plet representation-based algorithm is from the publicly available implementation developed by the original authors of the algorithm, Chikkerur et al. In order to ensure fairness in the comparison of the discriminating ability of the matching algorithms implemented as part of this thesis, a number of changes had to be applied to the original K-plet algorithm. Adjustments were made to the parameters of the algorithm to prevent it from using more distinctive information than the other algorithms had available. The only information available from the minutiae sets for all algorithms include the Cartesian coordinates of each minutia and the angle of the most closely associated ridge. As mentioned in [25] the source-code for the algorithm is available online. The only other significant changes required involved the usage of the developed XYT/MIN parser.

\subsubsection{Local-Scheme: Bozorth's Algorithm}

The last algorithm used in the fingerprint recognition system that we developed is the algorithm devised by Bozorth from the NIST. This was created as part of their NBIS package for aiding in the production of standards in fingerprint identification/verification technology. This algorithm is known as "bozorth3", "the Bozorth algorithm" or "Bozorth's Algorithm" and is a local-scheme.

The bozorth3 algorithm was developed as part of a demonstration to be used in the National Crime Information center [39]. Although it was originally intended only for demonstration purposes, it proved to be an effective method of distinguishing fingerprints, and is now popularly used as a benchmark for fingerprinting technology [39].

The only features which are used by the bozorth3 algorithm are the Cartesian coordinates and the angle of the most closely associated ridge. This makes it another good candidate for benchmarking the performance of the novel technique developed as part of this thesis.

The steps involved in the algorithm are the following [39]: 
1. Tables are constructed for both the query and database fingerprints. These tables contain feature information about each minutia relative to all other minutiae. These tables are referred to as "Intra-Fingerprint Minutia Comparison Tables".

2. From these two tables, a new "Inter-Fingerprint Compatibility Table" is constructed. Feature information which was found to be compatible between the two Intra-Fingerprint Minutia Comparison Tables is encoded within the InterFingerprint Compatibility Table.

3. The Inter-Fingerprint Compatibility Table is then traversed. The Inter-Fingerprint Compatibility Table is analogous to a "compatibility graph", where the associations between the two fingerprints represent edges in the graph. The longest chain of these is used as the matching score of the fingerprint.

The feature information which is included in the Intra-Fingerprint Minutia Comparison Tables is related to the relative position and $\theta$ values of each possible minutia pair. For each pair, six different measurements are used, which are the angle between each minutia's most closely associated ridge and a line-segment drawn between the two minutia, the angle between the line-segment and the horizontal axis, the Euclidean distance between the two minutiae, and the Cartesian coordinates of the two minutiae [39].

The traversal in Step 3 can be achieved using an interesting approach to solve some of the problems inherent with the compatibility graph constructed through invoking Steps 1 and 2. Some of the issues which the designers had to overcome have been outlined in [39], for example:

- The graph is very disjoint, consisting of many clusters which consist only of a single edge connecting two verticies.

- The maximum potential number of edges for each node is very high, and grows with the number of minutiae.

- The graph has the potential of being cyclic. 
Several more issues associated with the algorithm are listed in [39], but the above stand out as being the most difficult ones which have to be overcome. The solution created by Bozorth (reported in [39]) is to begin the traversals of the graph from a number of different starting locations, and creating additional edges between different nodes as the algorithm progresses through the graph. The additional edges form clusters of nodes which are combined in a consolidation step, resulting in a set of unique clusters. The number of linked nodes in the clusters is used as the match score [39].

\subsection{Chapter Summary}

In this chapter we have discussed several different pattern matching methods used in the fingerprint matching field of study, as well as discussed all but one of the pattern matching methods used in the fingerprint matching system developed as part of this thesis. 


\section{Chapter 6}

\section{Subsequence-Tree-Based Methods}

This chapter includes the relevant background information as well as the design process/rationale of a novel pioneering algorithm developed as part of this thesis. This novel strategy to fingerprint matching was developed as a method of evaluating the algorithm reported in [5], which itself was presented as a solution to the problem of recognizing the source of noisy subsequence trees. We have adapted this for the fingerprint matching problem.

First we will introduce and define the noisy subsequence tree recognition problem (NSTRP). Subsequently the similarities between the NSTRP and the fingerprint matching problem will be discussed. Finally, we will develop and evaluate a strategy for adapting the noisy subsequence tree recognition algorithm (NSTRA) to the fingerprint matching problem.

\subsection{Noisy Subsequence Tree Recognition Problem}

The basis of this new approach to fingerprint matching, especially in noisy environments, is the noisy subsequence tree recognition algorithm developed by Oommen et al. [5]. This algorithm was developed to solve a particular problem best described by way of an example [5]:

Suppose we have a large database of ordered trees, $H$. Let $X$ be an arbitrary 
tree from $H$, and $U$ be an arbitrary subsequence-tree obtained by randomly deleting nodes from $X$. The resultant tree (called a subsequence-tree or SuT of $X$ ) is further subjected to substitution, insertion, and deletion errors yielding the Noisy subsequence-tree (NSuT), $Y$. Our aim is then to identify the original tree, $X$, by processing $Y$.

Since the noisy tree $Y$ was produced, not from the original tree itself, but from a subsequence-tree, it should be compared against the subsequence-tree from which it was created. Such a solution strategy would add significant complexity, since the source tree of $Y$ is unknown, it would have to be compared with every possible subsequence-tree of $X$, which is, indeed, an exponentially large number of configurations. One method of counteracting this complexity is to include the use of information about the nature of the noise itself in the recognition of the original trees [5]. In order to accomplish this, the problem is parametrized based on the number of relabellings or substitutions that are expected to occur, which is correspondingly based on the noise affecting the transmission channel. In the original paper, the possible source tree, $X \in H$, of a given noisy subsequence tree, $Y$, is determined using the technique displayed in Algorithm 8. This algorithm uses the constrained edit distance calculated using Algorithm 9, which, itself, uses the Const_T_Wt array produced by Algorithm 10. The details of Algorithms 8 to 11 are omitted here to avoid repetition. They can be found in the original paper by Oommen et al. [5].

\subsubsection{NSTRP and Fingerprint Matching Problem Similari- ties}

The original goal of the noisy subsequence tree recognition algorithm was to effectively match subsequence trees to their source trees despite their having been subjected to a significant amount of garbling. A model of this problem which was utilized by the authors of [5] was the following: A "transmitter" sends a tree $X$ over a noisy channel; but prior to sending it, the "transmitter" deletes random nodes from the tree, thus producing a subsequence tree $U$, which is subsequently transmitted through the noisy channel, producing a noisy version of $U, Y$. The goal of the "receiver", who 

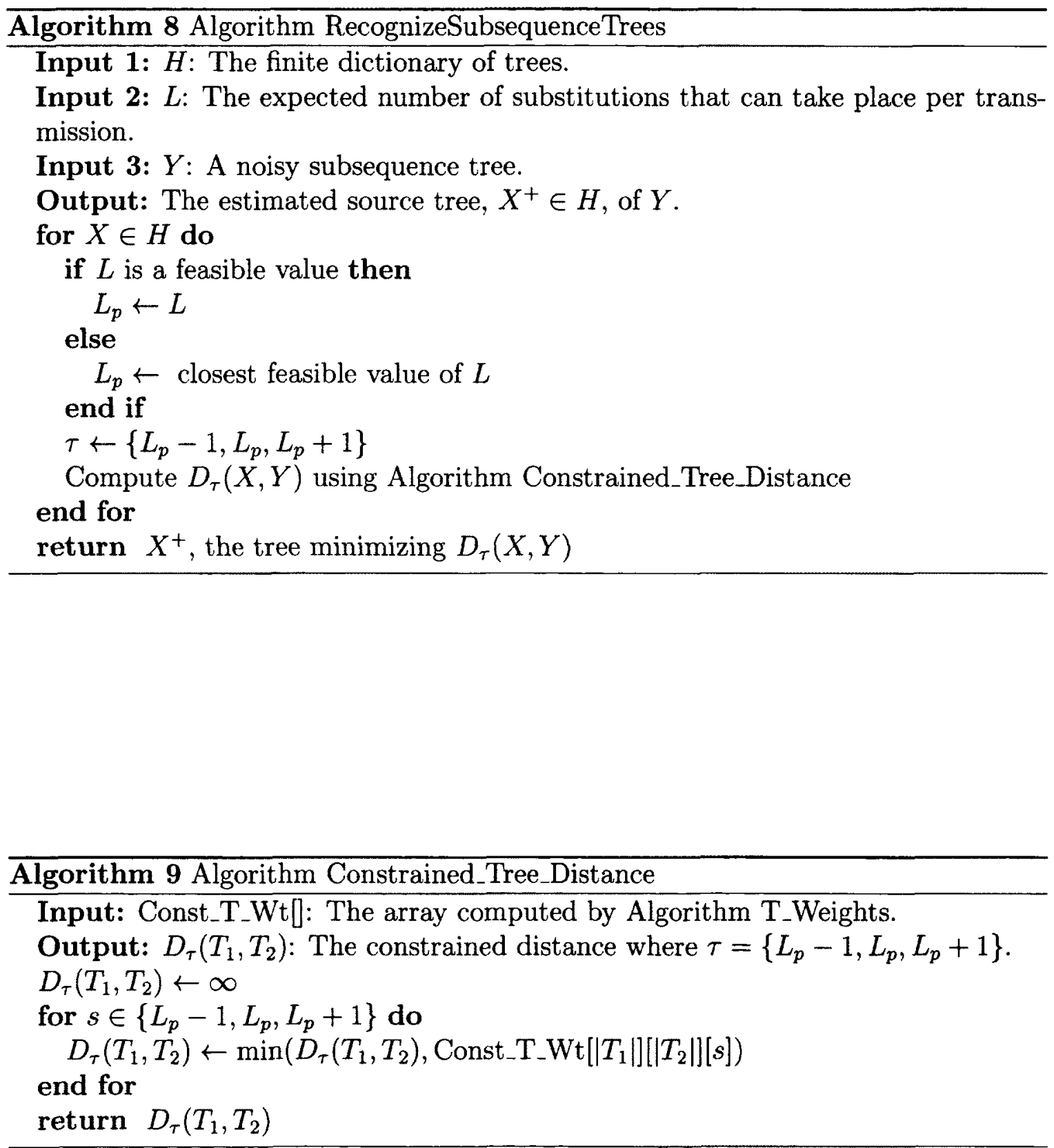


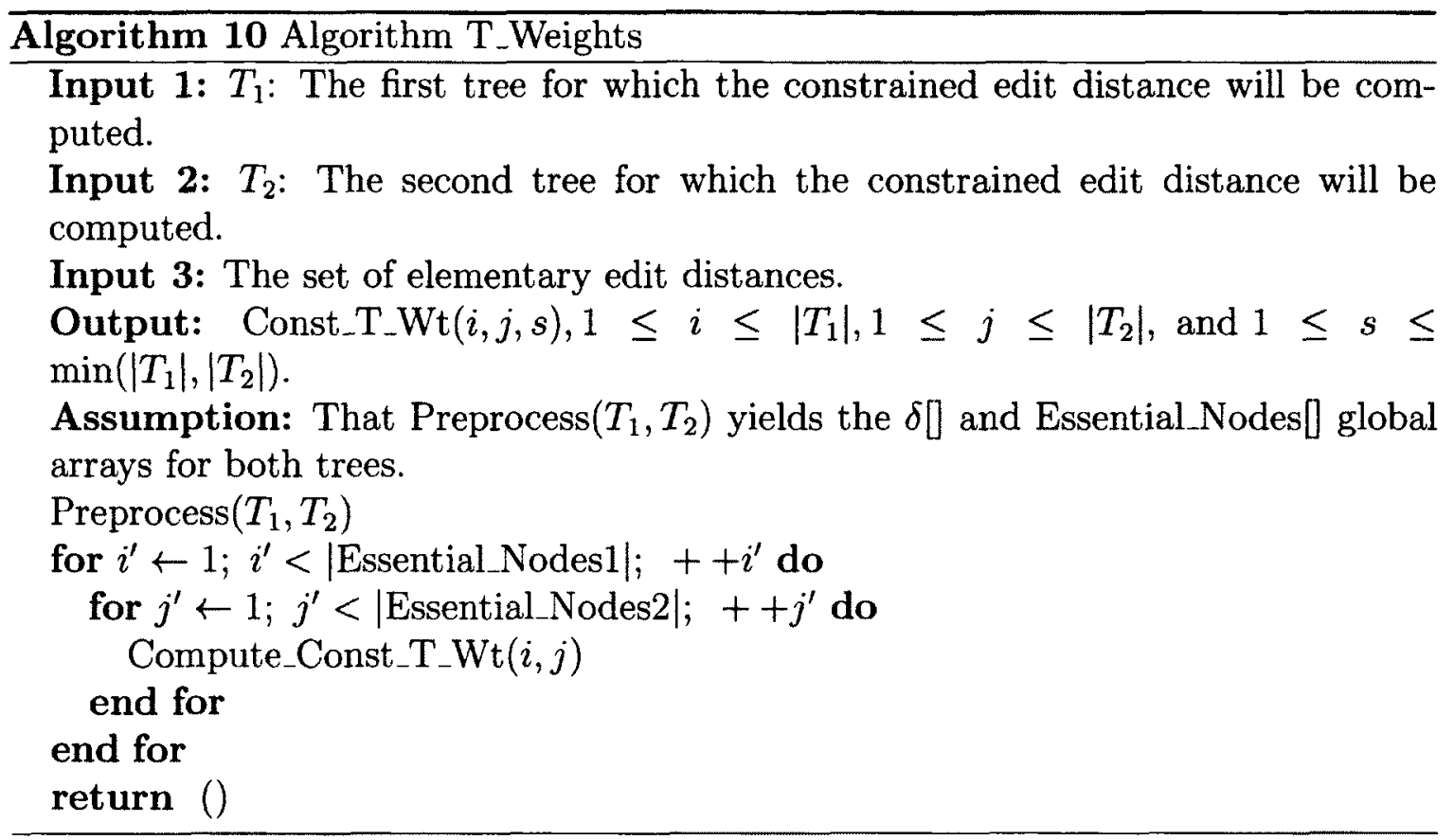

possesses a dictionary containing $H$, is to identify the source tree, $X$, from the garbled subsequence tree, $Y$.

The corresponding fingerprint matching problem can be stated as the determination of the source digit of a noisy and potentially fragmented fingerprint. Striking similarities between the two problems are immediately evident. Both problems attempt to achieve recognition of a source pattern from what is essentially symbolic data which may have had portions of the pattern removed, and which has thereafter been subjected to noise. As a result of the similarities between the two problems, it is obviously desirable to adapt solutions reported for the original problem to aid in the solution of the fingerprint related problem.

In practice, the collection of "latent fingerprints" often result in large portions of the original fingerprint image being unrecoverable. These unrecoverable regions leave mere fragments of the original fingerprint for the feature extraction and matching algorithms. This is, obviously, an extremely difficult problem, and conceptually it shares several common aspects with the noisy subsequence tree recognition problem. 


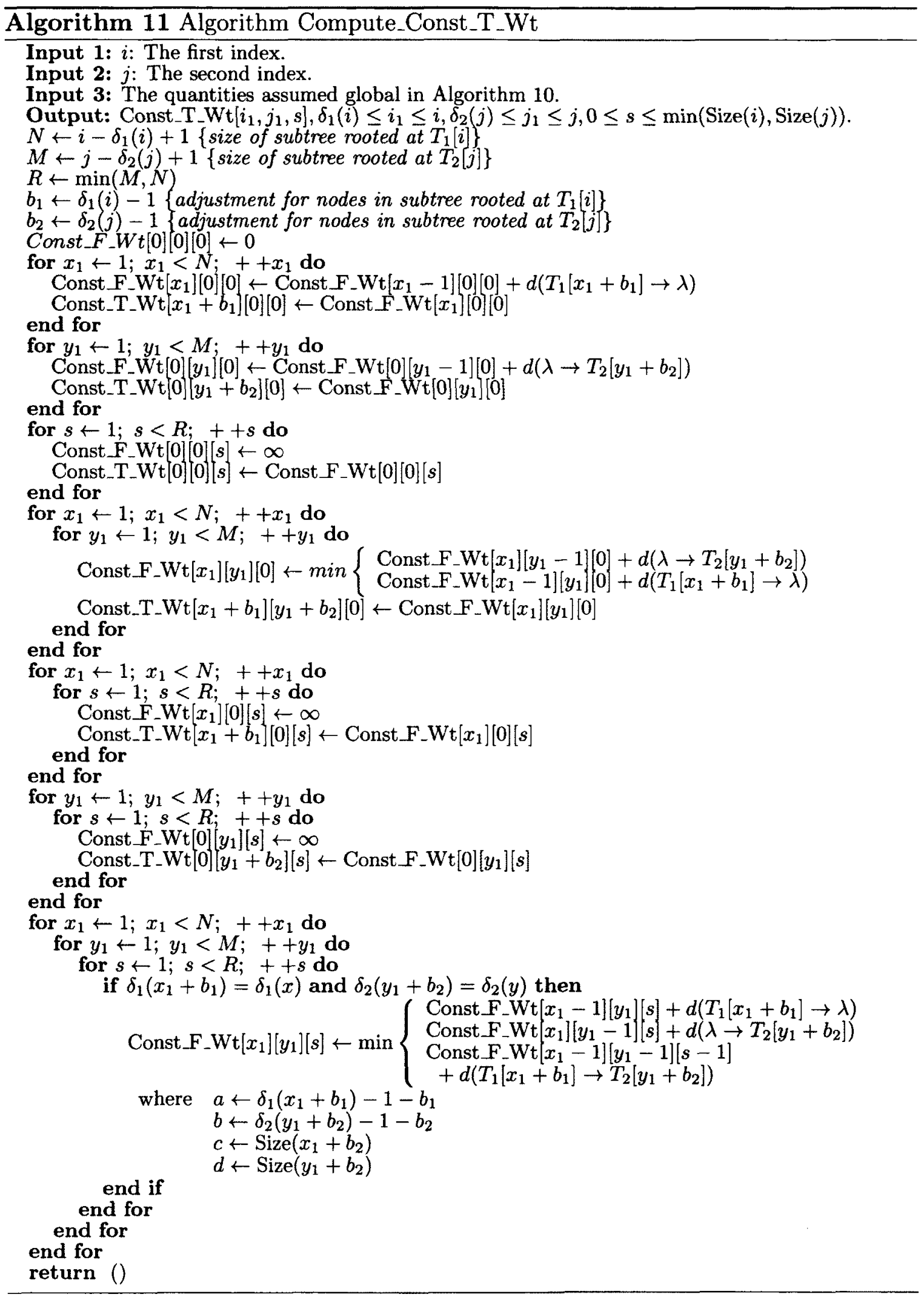




\subsection{Adapting Noisy Subsequence Tree Recognition}

The algorithm reported in [5] is a general purpose pattern recognition technique that can be applied to several different application domains. The fingerprint recognition (identification/verification) problem has the potential of being one of applications. The algorithm for determining the source pattern for a noisy subsequence tree can be regarded as a black box tree matching algorithm with high tolerance for noise in the query subsequence tree.

The algorithm's reported robustness in the presence of large noise renders it to be an ideal candidate for research in the area of latent fingerprint recognition. Recovery of latent fingerprints often leads to large portions of the fingerprint being unusably noisy. As already noted, this is analogous in spirit to the NSTRP in which unrecoverable information is randomly deleted from the fingerprint. The resultant fragmented representation is further perturbed by the noise inherent in the fingerprint matching problem.

The algorithm computes the so-called constrained edit distance between the original tree and the source tree, by taking into account what is known about the nature of the "noisy channel" causing the errors. In order to utilize the algorithm in the fingerprint matching problem, we must encode enough useful information from each minutia and their local-spatial relationships into trees, which can then be matched by the noisy subsequence tree recognition algorithm.

The task of encoding the minutiae and their local-spatial relationships into a tree necessitates developing a method for symbolically representing a portion of this information as the contents of the nodes of the tree, as well as determining how the parent-child relationships between each of these nodes is generated. Ultimately we must ensure that enough information is encoded within the tree's structure to accurately match trees generated from the same finger. Both of these key design decisions also require that the chosen features with which the trees are constructed are resistant enough to noise so as to permit the stable encoding of their information into trees. Choosing stable features ensures that the propagation of the instability of known noisy features is kept to a minimum. 


\subsubsection{Encoding Minutiae and Their Local-Spatial-Relationships}

The most important part of applying the noisy subsequence-tree recognition algorithm to the fingerprint recognition problem is to determine a method of encoding the minutia information in a tree of symbols which can be used by the algorithm.

Instead of dealing with the complexity of global-style fingerprint identification or verification algorithms, in this work, we designed a local-matching scheme. Using a local-matching philosophy implies that a structure would be created for each minutia, and avoids the issue of creating a global tree, which would necessitate creating a stable method of choosing the root node of the fingerprint's tree representation. Instead, one tree is constructed for each of the minutiae in the set extracted from the fingerprint.

In addition to being a local-matching technique, we decided that in order to avoid adding unnecessary complexity to the algorithm, the local structures should be rotationally independent. This also has the benefit of improving the algorithm's resistance to rotational noise.

Each tree is composed of symbols, the nodes, and the parent-child relationships between the nodes. The ultimate performance of the algorithm hinges on the decision as to which information should be encoded into the symbols and the parent-child relationships of the symbolic trees.

In our solution, the angle of the most-closely associated ridge, relative to that of the root node of each minutia, is used to create the symbol which represents that minutia. The angle is discretized into 20 different symbols $\phi_{1}, \ldots, \phi_{20}$. The minutia which is the root of the tree is always set to $\phi_{1}$ (a relative $\theta$ of 0 ), and subsequently the minutiae from the root's local region have their representative symbol determined by calculating the relative angle between their $\theta$ angle and root's $\theta$.

The minutiae which are considered part of the root's local region are those which are within a radius $r$. The levels of the tree are defined by linearly increasing thresholds of the Euclidean distance between the children minutiae and the root. A way by which this can be modeled is by picturing a series of concentric circles surrounding the central minutia, as shown in Figure 6.1. Each band in the concentric circles represents another level of the tree. The parent of any minutia $m$ can be defined as the minutia $m_{\text {parent }}$ which is contained within the next smallest concentric circle closer 


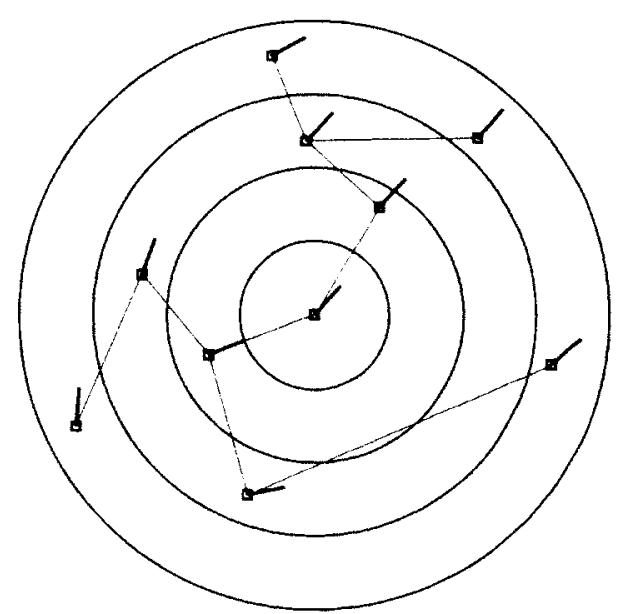

Figure 6.1: Diagram illustrating the concentric circles which define the levels of the minutia tree. The minutiae are highlighted with red squares, the angle of the most closely associated ridge is indicated with a blue line, and the child-parent relationships are shown in green.

to minutia $m$. Any band of the concentric circles which is empty is joined with the next smallest circle so as to prevent the creation of an empty level of the tree. This is illustrated in Figure 6.2. The exact method used to construct the trees is shown in Algorithm 12.

The determination of appropriate values for the MAX_RADIUS and BAND_WIDTH constants was accomplished by manually adjusting the values and observing the size of trees produced for several test fingerprint minutiae sets. The minutiae tree generation algorithm was tuned until the average tree size was 15 nodes with the values of MAX_RADIUS and BAND_WIDTH set to 120 and 10 respectively.

Once a tree has been constructed for each minutia, a vector of the query fingerprint's trees is compared against a vector of the database fingerprint's trees. Comparison is achieved through the usage of the noisy subsequence-tree matching algorithm, which results in a constrained edit distance. The best-match (one with the lowest edit distance) is used for each tree to match the minutiae together, which results in a vector of matched minutiae, $M$. The size of the vector $M,|M|$, is used to calculate the match-score between the two fingerprints in a similar manner to that of the other 


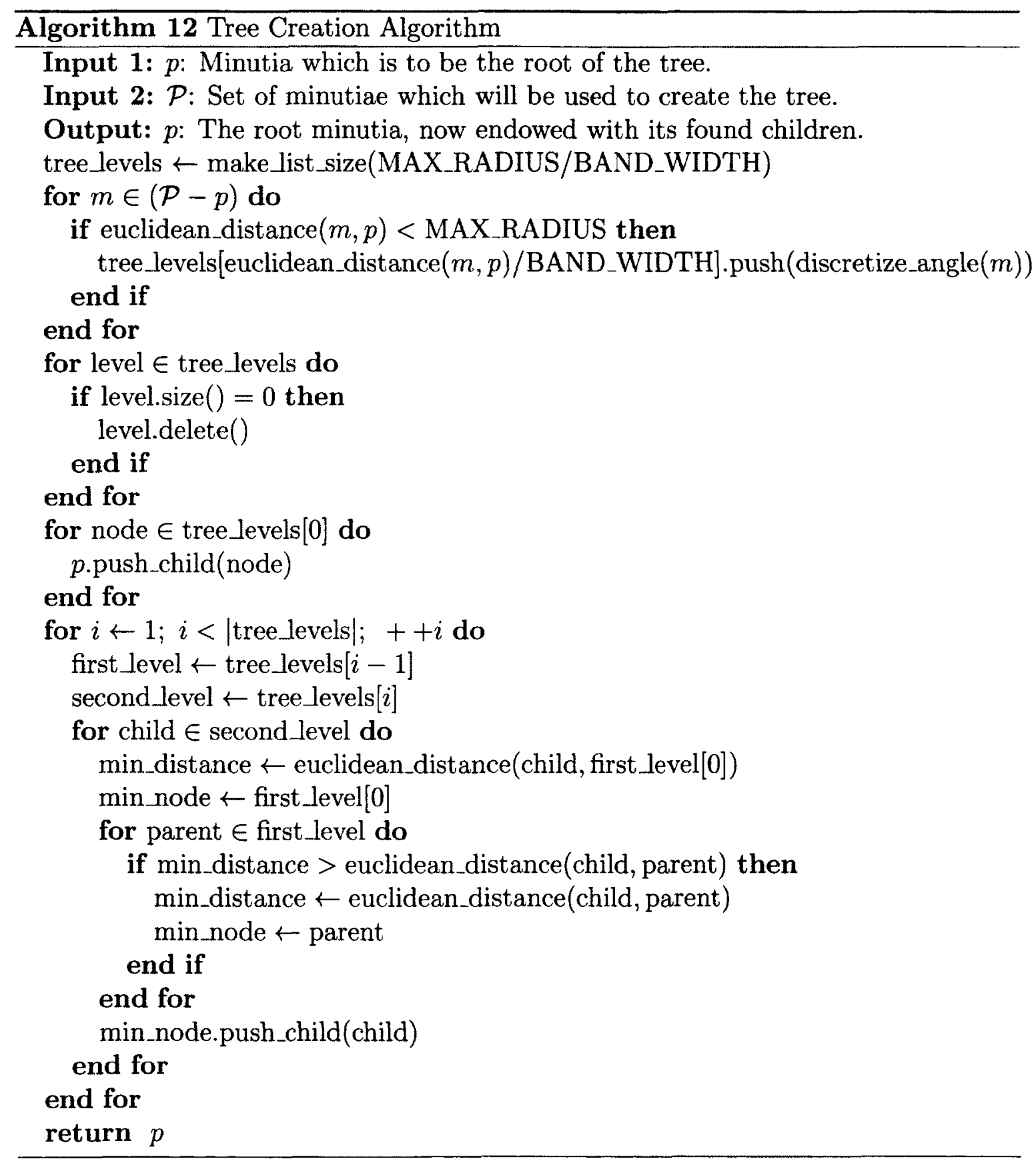




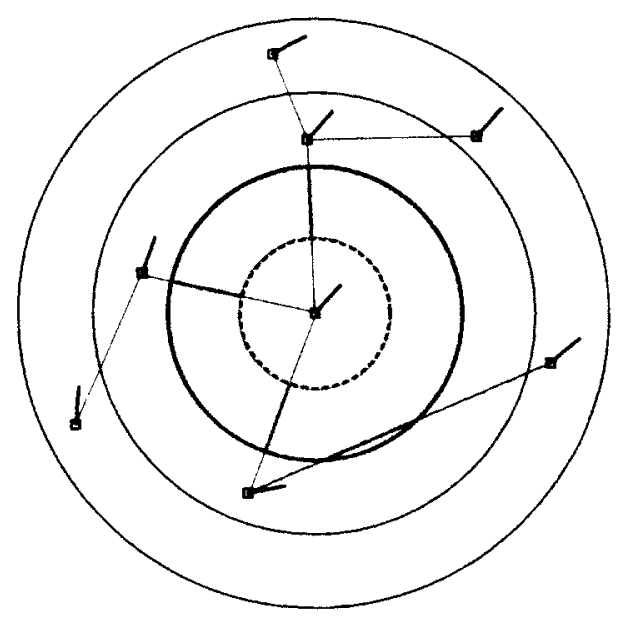

Figure 6.2: Diagram illustrating what occurs when one of the bands formed by the concentric circles is empty. The empty band is highlighted in pink, and is joined with the next smallest concentric circle indicated with a dotted line. These two now form the first level (root) of the tree.

fingerprint matching methods described in Chapter 5. This is shown in Eq. (6.1).

$$
\text { score }=\frac{|M|^{2}}{|\mathcal{P}| \cdot|\mathcal{Q}|}
$$

\subsubsection{Noise Considerations}

The last task required to fully adapt the noisy subsequence recognition algorithm to the fingerprint recognition problem is to incorporate knowledge of the nature of the noisy channel. In the case of fingerprints, the "channel" includes everything from the fingerprint scanner, occlusion, and then to the minutia-extraction mechanism. This can be incorporated by adjusting the parameters provided to the noisy subsequence recognition algorithm.

Although the algorithm has been tested in its current state, it is incomplete when it concerns the full adaptation of the NSTRA to a specific fingerprint system. The reader will observe that the primary purpose of the assumption parameters of the noisy subsequence tree recognition algorithm are to provide it with an approximation of the number of edits the algorithm is permitted to use to transform one subsequence 
into another complete tree.

In order to choose the appropriate noise parameters for the algorithm, the noise inherent in fingerprint recognition systems must be studied and understood. There are a number of different sources of noise as already described in Chapter 2. In summary these are:

- Rotation.

- Displacement.

- Distortion due to skin plasticity.

- Variance in pressure when the print is taken.

- Use of older fingerprint images.

- Differences in fingerprint collection techniques.

- Noise due to equipment operation or failure.

These sources of noise are modeled as affecting the unique subsequence fingerprint as it is propagated through the "noisy channel", and which ultimately result in insertions, deletions, and substitutions in the tree representations of the minutiae. A strategy for approaching this problem is elaborated upon in the discussion contained in this Chapter.

\subsection{Subsequence Algorithm Performance}

Two main issues had to be taken into consideration when performing the experiments to measure the performance of the developed algorithm. These involved eliminating bias and mitigating the prohibitive runtimes involved.

Separate data was used for the training and the subsequent experimentation. This ensured that there was no bias that resulted from the process of manually tuning each of the algorithms. 


\begin{tabular}{|l|c|c|c|c|}
\hline Algorithm & Accuracy & Precision & Recall & FPR \\
\hline Bozorth's Algorithm & $99.7 \%$ & $98.7 \%$ & $74.8 \%$ & 0.00009 \\
Hough Transform & $99.3 \%$ & $97.7 \%$ & $34.5 \%$ & 0.00007 \\
K-plet & $92.9 \%$ & $10.1 \%$ & $76.8 \%$ & 0.07267 \\
NSTRA-based & $74.6 \%$ & $2.7 \%$ & $69.8 \%$ & 0.17482 \\
\hline
\end{tabular}

Table 6.1: Table outlining the overall Accuracy, Precision, Recall, and false positive rate of the implemented/procured algorithms.

In order to deal with the prohibitively high runtimes of "full runs" of the algorithms against the database of fingerprints ${ }^{1}$, a subset of cardinality 800 of the full database was used for the experimentation. Also, in order to mitigate, as much as possible, the influence on the results by our selection process, random images were selected from the database to form this subset, and the same random subset was used to test each of the fingerprint matching algorithms.

There are a number of metrics which can be used to compare the relative performance of one matching algorithm with respect to another. Ultimately, the performance of a particular fingerprint matching algorithm is highly dependent on the quality of the fingerprints in question. The variance in the quality of the fingerprint, as well as in the type of noise or damage afflicting a particular fingerprint, may have a great impact on which fingerprint recognition algorithms perform best.

The overall percentage of correct matches produced by the algorithm, as well as the false positive rate (FPR) metrics from the experiments are displayed in Table 6.1. Implications of the results are discussed in Section 6.4.1.

\subsection{Using Artificial Noise}

As the NSTRA-based algorithm is fairly general in its scope, it is obviously desirable to attempt to determine the discriminating power of the algorithm in its complete form. The only portions of the algorithm which remains incomplete are the noise

\footnotetext{
${ }^{1}$ Matching each fingerprint against the others would require 7,040 $\times 7,040$ comparisons, each approximately a second in length, is a total runtime of approximately 573 days.
} 


\begin{tabular}{|c|c|c|c|}
\hline Accuracy & Precision & Recall & FPR \\
\hline $98.1 \%$ & $5.1 \%$ & $81.7 \%$ & 0.02030 \\
\hline
\end{tabular}

Table 6.2: Table outlining the overall Accuracy, Precision and the false positive rate of the NSTRA-based algorithm when used on the manually garbled trees.

considerations which have been simulated in order to evaluate the approximate discriminating power of the algorithm.

In order to simulate expert knowledge of the characteristics of the noisy channel, trees generated by the our tree generation algorithm were garbled using a method with known properties. Noisy trees generated from this garbling algorithm were matched against a database of the minutia trees generated by the method described in Algorithm 12 from the same 800 fingerprints which were used in the previous experiments.

The NSTRA-based algorithm was then used to search for the source fingerprint of each set of garbled trees. It should be noted that this is a similar but slightly different problem to that of finding the source finger of a set of fingerprints and is not an entirely accurate representation of the fingerprint recognition problem itself. However, we argue that it is still useful for determining the discriminating power of the algorithm in a situation where the properties of the noisy channel (in our case the fingerprint recognition system) are known.

The results obtained from this experiment are displayed in Table 6.2.

\subsubsection{Results Analysis/Future Work}

There are a number of metrics describing the performance of the fingerprint recognition algorithms provided in Tables 6.1 and 6.2. The most important results for a fingerprint identification system is the measurement of the algorithm's Recall rate. It is important that the recognition algorithm does not discard any fingerprints which are, indeed, generated from the same digit, because, finally, an identification system's results are intended to reduce the manual workload of a fingerprint identification 
expert. Accuracy is also important, but this is greatly influenced by the ratio of fingerprints corresponding to the probe fingerprint, and the total number of fingerprints in the database. Since the total number of fingerprints in the database is often far greater, the difference in Accuracy between different algorithms will appear to be small. The Precision of the fingerprint recognition algorithm is also important, as it indicates, in part, how many correct results will be contained within the returned set of fingerprint matches.

Bozorth's Algorithm performs as reported in [39], with an overall Recall of approximately $74.8 \%$ and with a very low false positive rate.

The Hough Transform-based algorithm does not perform particularly well overall, but it is capable of matching fingerprints of high quality. This is as expected because it is a relatively simple technique, and large quantities of noise prevent the algorithm from performing useful transformations on the minutiae "point sets". During the tuning process it was noted that the Hough Transform alignment had difficulty producing a suitable alignment for performing a point-to-point match once a modest levels of noise was reached. This could explain its poor Recall when challenged with levels of noise found in the fingerprints in the FVC2000/FVC2002 databases.

The K-plet-based algorithm performs excellently, obtaining an overall Recall of $76.8 \%$. However, it has a low Precision rating of $10.1 \%$, which results in a slightly larger returned sets of fingerprint matches. Because of the local-scheme nature of the algorithm, the K-plet-based algorithm has a much higher false positive rates than either the Bozorth's Algorithm or the Hough Transform-based algorithm.

The NSTRA-based algorithm obtained a Recall rating of $69.6 \%$ which is comparable to the algorithms it was competing with, although the false positive rate (FPR) was much higher. There are several possible reasons for the high FPR. The NSTRA-based algorithm is a local-scheme and is thus much more tolerant of distortion and has a much lower discriminating power than a global-scheme algorithm [1]. Also, as previously mentioned, the NSTRA-based algorithm, as it has currently been implemented, has not yet fully incorporated the information about the noise in the fingerprint's channel, which we believe, could be a source of both inaccuracy and 
the source of the high false positive rate. There are also consolidation steps incorporated into both Bozorth's Algorithm, and the K-plet-based algorithm which could be significantly lowering their false positive rates.

In spite of its current lack of proper noise parameters for an applied fingerprint recognition system, we believe that it does show some promise as a technique for matching fingerprints. The most concerning point is the extremely high FPR and subsequently it's low Precision rating.

A number of strategies can be used to possibly lower the FPR, such as that of using the well-known consolidation techniques for local-scheme algorithms. One common technique for consolidation is to iterate through the matched pairs of local structures and to use the central or source minutia of each of them to perform further evidence gathering. Evidence of shared origin of the two structures could be gathered from any features known to be stable.

\section{Accuracy in the Simulated Environment}

The simulated "parametrized" NSTRA-based algorithm performed much better than that of the "un-parametrized" version with a Recall rating of $81.7 \%$ and a FPR of 0.02030 . Although these figures are considerably higher than that of the experiments using noise from a real fingerprint recognition system, we emphasize that the experiment performed to obtain these results is not fully representative of the fingerprint recognition problem. Nevertheless these results do, indeed, demonstrate that the algorithm has significant discriminating power and warrants further study.

\subsection{Chapter Summary}

In this chapter we have introduced the noisy subsequence tree recognition problem, and the solution to this problem reported in [5]. We have also developed an adaptation of this algorithm to the fingerprint recognition problem, as well as performed an evaluation of this novel pioneering algorithm as it pertains to fingerprint recognition. 


\section{Chapter 7}

\section{Overall System}

In order for the reader to get a better picture of the project that we undertook, it is important to overview the components of the system developed as part of this thesis. This will also properly put into perspective the performance of each matching algorithm, by providing a full and detailed picture of the environment in which the algorithms were tested.

This chapter overviews the design and development process as well as the major features of the system developed throughout the course of this study. The major decisions made throughout the design will also be described and rationalized. The implementation details are provided for each of the phases described in Chapter 2 (Section 2.6).

An illustration of the various parts of the system, as well as the state of the data/information on its path through the system is given in Figure 7.1. The light blue lines represent the flow of data through the system, and the red dotted-lines represent manual redirection of the data-flow by its human supervisor.

The detailed overview that follows includes the design rationale, the main features of the system, and the system's components as they pertain to the phases described in Chapter 2. To illustrate the available interface some typical examples are also included. 

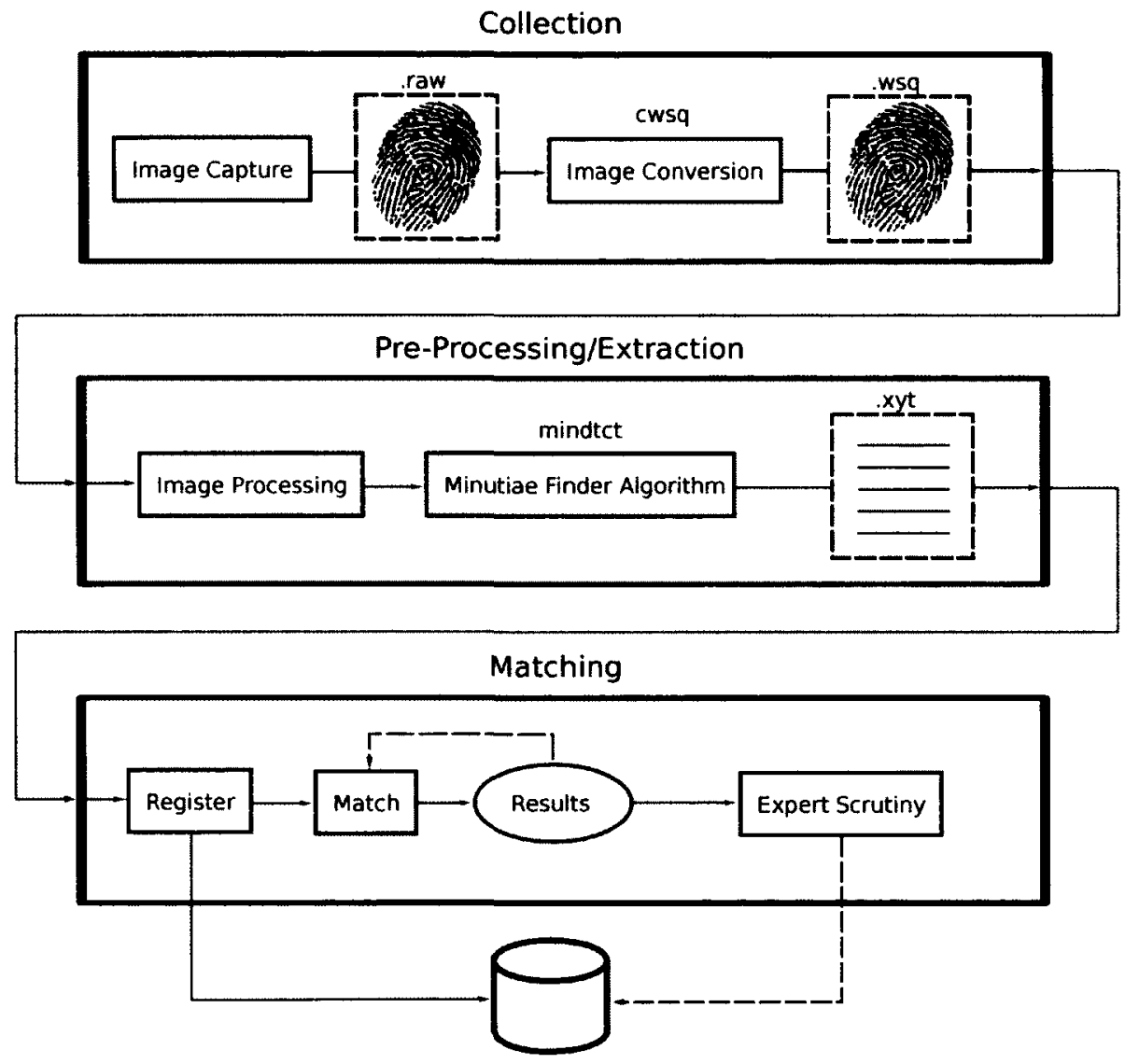

Figure 7.1: Diagram illustrating the overall system, shows each stage and the filetype/state of the information as it moves through the system. 


\subsection{System Features}

First of all, it is pertinent to mention that the fingerprint recognition system was developed keeping in mind specific fingerprint matching algorithms. We emphasize though that we have incorporated built-in tools for the benchmarking of biometric matching algorithms, including capabilities for adding noise, adding new algorithms, and piping the results from one phase to another. In particular:

- Fingerprint matching can be performed with a number of different algorithms including the Bozorth algorithm, a Hough Transform-based algorithm, a K-plet representation-based algorithm, and a novel noisy subsequence tree recognitionbased algorithm.

- The system is not limited to the base set of matching algorithms. It is capable of being easily extended with new techniques. Extensions can be added via Python's system shell utilities or through the use of the "ctypes" extension library for linking shared object files.

- Matching sets of prints can be fed from one algorithm to another, permitting the construction of hierarchical matchers.

- The system components are all scriptable and have easy-to-use APIs.

\subsection{Design Process/Rationale}

A fingerprint recognition system, whether it is designed for identification, verification, or both, is rooted in the transformation of $\mathrm{RAW}^{1}$ images collected from a fingerprint scanner to a matching process between those RAW images. Thus, the ultimate goal throughout the design of a fingerprint recognition system is to create the data pipeline from the fingerprint scanning stage to the matching stage.

Although a complete system should have been able to collect fingerprints, it would be impractical to collect a large enough set of fingerprints for testing when they are

\footnotetext{
${ }^{1}$ When referring to something as "RAW" (with all capital letters) we are not indicating that it is raw data, but rather information in one of the several existing raw image file formats.
} 
easily available elsewhere, and thus the first task was the collection of fingerprints from existing fingerprint databases. In addition, we set out to obtain, from open source software, as many of the components of the system as possible so as to not repeat previously completed public efforts.

With respect to data collection, we were able to obtain access to two fingerprint databases from the FVC2000 and FVC2002 international fingerprint verification competitions. These databases, collectively, included 7,040 individual fingerprint images from a total of 880 unique digits. Each of these images were stored in the widely used TIF image format.

Of the open source software components available in the fingerprint recognition field, the toolkit from the NIST was determined to be the most comprehensive one. The NIST toolkit had the additional advantage of explicitly not requiring a license. This NIST fingerprint recognition software toolkit is known as the NIST Biometric Image Software (NBIS) package. The tools included as part of the NBIS package are primarily designed for working with RAW, WSQ, and JPEG images. In order to take advantage of as much of the NBIS package's tools as possible, we needed to be able to convert between the TIF format of our procured databases of images to formats supported by the package.

The tool we were most interested in from the NBIS package was the "mindtct" program used to extract minutiae from fingerprint images. In order to use this program, we needed the ability to convert our TIF database into the FBI's standard biometric WSQ image format. Tools for converting, both from and to, RAW and WSQ images were included with the NBIS package, namely "cwsq" and "dwsq". Although these were programs for encoding and decoding WSQ images respectively, they were not immediately usable. In order to utilize the NBIS package with our system we needed to determine which RAW format the NBIS package supported. A number of different RAW formats exist for raw image data, of these we determined that the RAW image format used by the NBIS package was a plain one-dimensional array of bytes which could be read directly into a c-style string and converted to-and-from various formats by using tools like the Python Imaging Library. Therefore, we converted the TIF images into the NBIS package's RAW format, which permitted the use of the "cwsq", 
"dwsq", and "mindtct" programs.

Once the complete usage of the NBIS package was achieved, much of the image conversion, image processing, and minutia-extraction phases of the data pipeline in Figure 7.1 could be constructed.

\subsubsection{Fingerprint Library}

The completed components necessary for constructing the data pipeline, as outlined in Chapter 2 (Section 2.6), and illustrated in Figure 7.1, were conglomerated into a cohesive and consistent wrapper library. In addition, this library contains useful abstractions and features not provided by its constituent components.

We adopted an object-oriented approach which was centered around an abstraction over all the files and processes that provided the pre-match information about a fingerprint. The information we refer to here is predominantly that which is used by various matching algorithms. This also includes the file formats: RAW, WSQ, TIF, PNG, XYT, and MIN. A fingerprint object was designed for the tracking of a particular fingerprint's files including its RAW, WSQ, PNG, XYT, and MIN files. The TIF format was excluded since the fingerprint databases were converted entirely into the FBI's WSQ format. The resulting fingerprint class is outlined in Figure 7.2. Fingerprint objects would track the location of each of the files associated with the individual fingerprints without needing to produce them or load them into memory until it was absolutely necessary ${ }^{2}$, as well as being able to take an image of any of the supported types and producing the rest of the files on-demand.

The primary functions for manipulating the fingerprint objects are the following: move, match, wsq, xyt, min, image, register. With these functions available, a developer can move the set of files representing fingerprint data all at once, register the data in the database, request that a file and its associated data be generated, or match that fingerprint against another with an existing or new algorithm entered in "extensions.py". With respect to the components described in Chapter 2 as well as the components highlighted in Figure 7.1, the fingerprint object handles image

\footnotetext{
${ }^{2}$ Although the information, such as minutiae, are extracted lazily (on demand), they can also be loaded from a previous session.
} 


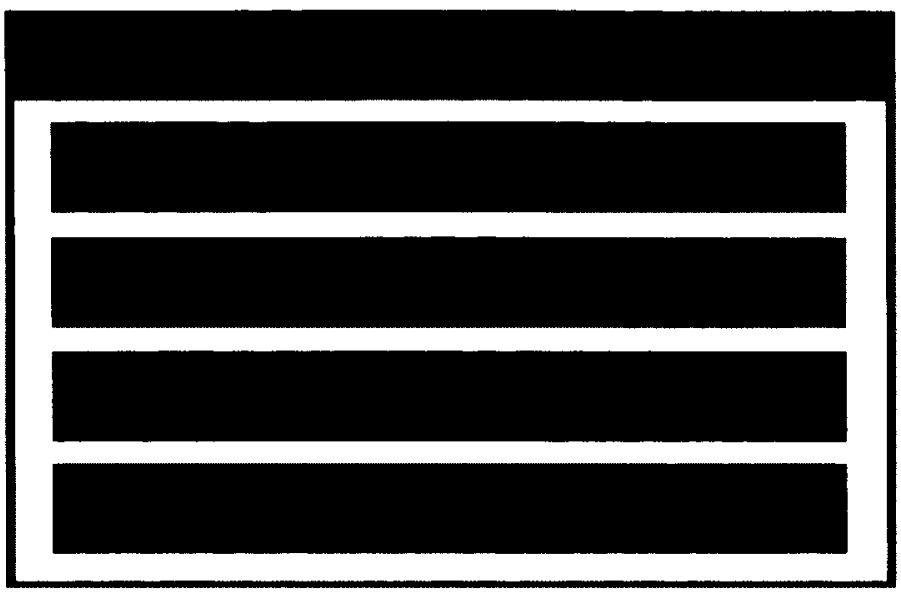

Figure 7.2: Diagram of the fingerprint class, showing the main externally-visible fields of the class.

conversion, image processing, minutia-extraction, and to a lesser extent, hides the complexity of dealing with matching utilities.

A developer should be able to use the library to create a fingerprint matching system either with the fingerprint matching algorithm provided by the NBIS package (bozorth3) or by supplying his/her own fingerprint matching algorithm. Extending the library to include more fingerprint matching algorithms is a matter of using the "ctypes" extension interface to call C shared object libraries or of running the matching utility by passing execution to the system shell, potentially requiring the placement of the algorithm on the system's "PATH" variable. Once fingerprint matching algorithms have been added, using them to match fingerprints would merely involve identifying the intended algorithm with a string argument to the fingerprint's match function.

\subsubsection{The Piping Mechanism}

The ability of the system to pipe results from one algorithm to the other was implemented by abstracting the pipe to a linked list of algorithm names. Each name is a unique string identifier of a fingerprint matching algorithm. Once a user has decided to perform a piping operation, the results will be passed from one algorithm to the 
next identified in the list.

Algorithm identifiers are created by the user or developer upon the addition of the corresponding algorithm to the global list of algorithms. Each fingerprint object stores the method by which it will be compared to other fingerprint objects.

This modular and flexible approach allows for both simple scripting by a developer as well as for simplifying scripting for an expert user. The effect of executing multiple algorithms in succession in this manner was not fully tested, but studies on its effects are available in the literature.

\subsubsection{Combined Components}

Once the library abstracting the disparate components of the system was completed, all that remained in order to complete a fully working fingerprint recognition system was the implementation of a relatively simple "1-to- $N$ " algorithm similar to that which is shown in Algorithm 13.

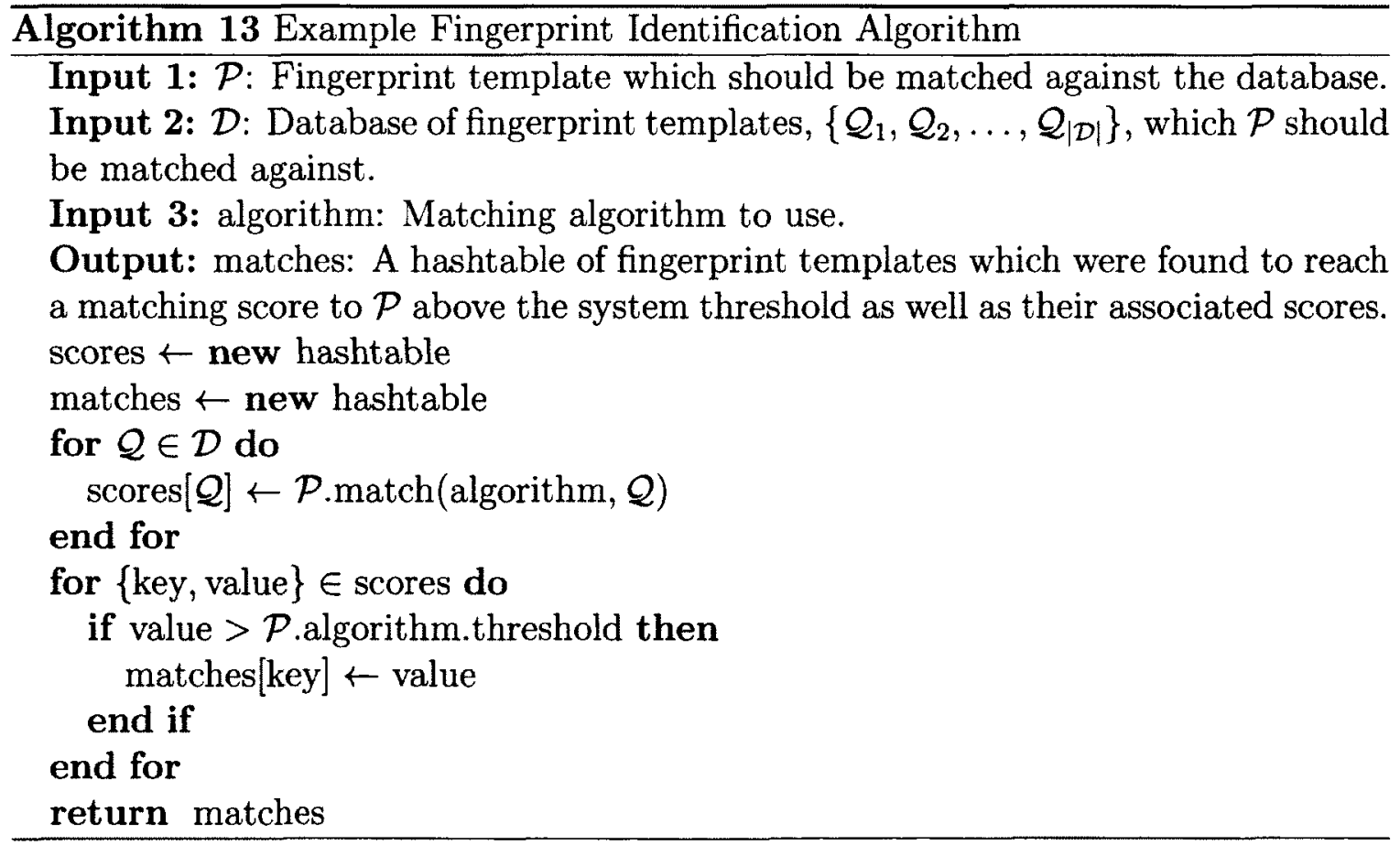


The schematic strategy shown in Algorithm 13 would work, and would be acceptable in a number of applications. However, there is a more elegant way to accomplish this same task using the built in piping mechanism shown in Algorithm 14. Both approaches have their inherent advantages: interacting with the system on an abstraction level similar to that of Algorithm 13 allows clarity while still being able to manipulate the matching process in a meaningful way. A common practice specific to the maintenance of a verification fingerprint matching system would involve the accommodation for special cases, for example, an unusually low matching score for particular individuals could be handled by adding a special case to the algorithm. A simple solution to this example problem would be to lower the matching score threshold when trying to verify the identity of this specific person. This could be easily implemented by the user/administrator using the abstraction level shown in Algorithm 13 without having to deal with the low level details of interacting with the fingerprint images and extracted minutiae themselves.

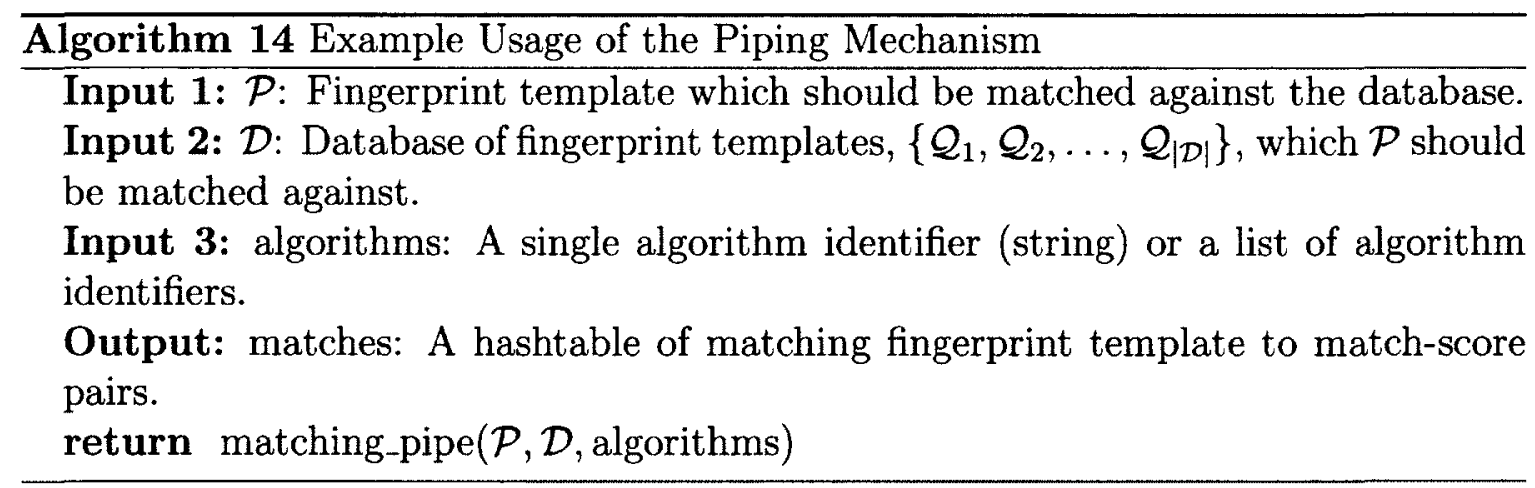

\subsection{System Components}

This section outlines the system components in the typical fingerprint recognition system and how they relate to the system that we have developed in this thesis. 


\subsubsection{Raw Data Collection Phase}

Raw data collection is an important phase in fingerprint matching systems, and support for it is included as part of the system. However, normal operation of the system that we have developed implies the use of a pre-existing database since using new raw data for consecutive tests would not provide enough statistical power to provide interesting results.

Raw data collection, when it is used, is accomplished through the use of a scanner. The fingerprint scanner outputs a RAW image file which can be converted into a WSQ image with the use of "cwsq" the WSQ compression program described above. Once the image has been converted into the WSQ format, it is added to the fingerprint database. An alternative digital fingerprint sensor can be used along with a set of libraries for interfacing with them. An appropriate open-source library for use with Linux and fingerprint scanners is the "fprint" fingerprint library, which is used in the described system.

For the purposes of experimentation, we have not utilized the option of using actual raw data collection. Rather, we have elected to use the same input WSQ format images for each test to ensure consistency and to avoid adding additional variables to the experimentation/test framework.

The data used in the system for the experimental analysis of the performance of each of the matching algorithms are from the international fingerprint competitions FVC2000 and FVC2002. In these sets, a collection of 7,040 fingerprints from the NIST's database were obtained from 880 unique digits.

\subsubsection{Data Enhancement Phase}

Although it is critical to a production environment, data enhancement is largely ignored in the developed system. This is partially because of the use of the NBIS package, which handles minutia-extraction straight from WSQ images, and partially as a result of the small effect that data-enhancement would have when comparing the relative performance of different fingerprint matching algorithms against eachother. In addition, extensive pre-processing of the image may add an unintended and 
undesirable bias to the performance of a specific algorithm.

The data enhancement that is present is part of the "mindtct" algorithm obtained from the NIST. The algorithm takes advantage of contrast adjustment for low contrast images. Quality mapping is also performed in order to obtain quality-scores for each extracted minutia so as to aid in the reliability of the respective algorithms.

\subsubsection{Feature Extraction Phase and Template Construction}

Feature extraction is obviously an essential component of the fingerprint identification and verification processes. The developed system is no exception, and takes advantage of the the widely used and open-source "mindtct" algorithm provided in the NBIS package. The feature extraction is performed by binarizing the enhanced image, using a fingerprint scanner to perform the actual collection of the minutiae.

The minutiae are extracted and presented along with the angle of their mostclosely associated ridge, the ridge count between them, their most-closely associated neighbor minutia, and their type. There are also a number of other qualities of the minutia which are stored upon execution of the "mindtct" algorithm but only the ones listed are used by the implemented algorithms. Two of the several files produced by the "mindtct" algorithm are used, which are the XYT and MIN files.

Once the system has extracted minutiae, the minutia data is stored in plain text files formatted with the XYT format used by the NIST minutia matching algorithms, as well as the MIN format produced by the "mindtct" program. The XYT files store each minutia as one line in the file, with the Cartesian coordinates and the minutia angles delimited by spaces. The MIN file contains significantly more information including ridge counts between nearby minutiae and the type of minutiae.

The templates themselves are actually the fingerprint objects described in Section 7.2.1. The most important information which is required by fingerprint matching algorithms resides in the its template which consists of two types of minutia information files: MIN and XYT. 


\subsubsection{Minutia Matching Phase}

Minutia matching is performed using one of the four different techniques developed and obtained during the research performed in the production of this thesis: Bozorth's algorithm provided by the NIST software package, the Hough Transform elastic matching technique, the K-plet graph-based matching technique, and a technique based on the matching of noisy subsequence trees.

As explained earlier, additional matching techniques can be added. All that is required to accomplish this is a "thin" layer of Python either through the "ctypes" foreign function interface or simply by a scripted call to the system shell. The matching algorithms can also be run in parallel by using a wrapper class for the "gnu-parallel" utility or with Python threads.

Algorithms can use either the XYT or MIN files to obtain minutia information. Although it is rare for a matching algorithm to work directly with the image itself, this option is also supported by the fingerprint object's references to the image files of its associated fingerprint.

Once an algorithm has been produced, it may be added to the system by editing the "extensions.py" script. The algorithm should take the file-names (one or more of the XYT, MIN, RAW, or WSQ files) it requires as arguments. The files are passed to the function through the use of the Fingerprint object and are accessible by named accessor methods. After the algorithm has an entry in the "extensions.py" file, it may be called by the command-line interface to the fingerprint identification system, or may be used in a script which uses the fingerprint systems libraries.

\subsection{Usage Examples}

In order to illustrate the usage of the available interface to our fingerprint recognition system as well as to give an overview of the options supported by the interface, some usage examples are included in this section. The format of the examples follows shell syntax ("\#" indicates a comment) and is also similar to that which is used in "man pages" where square-brackets indicate the location of a token to be supplied by the 


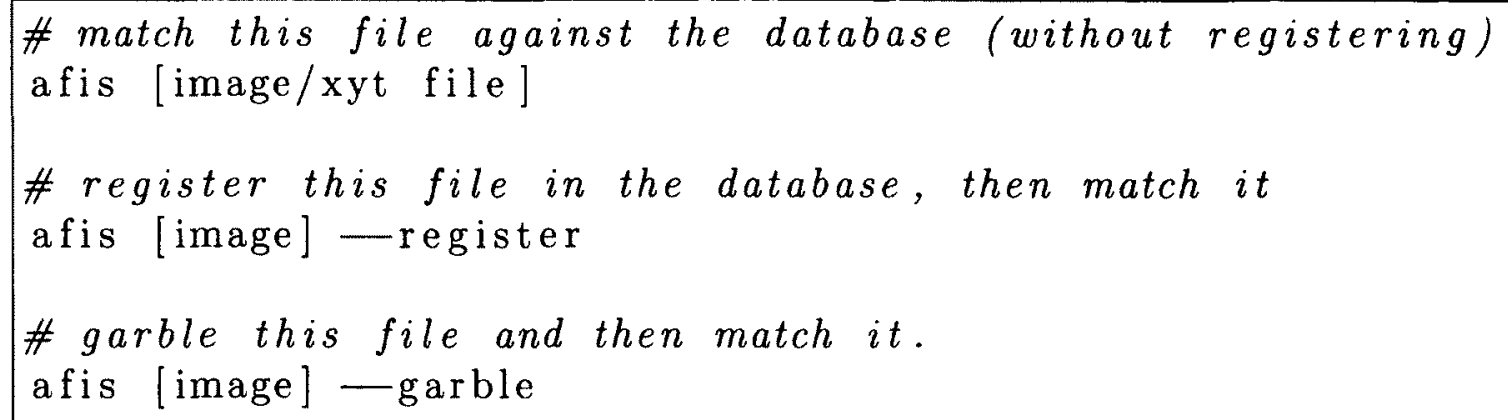

Figure 7.3: Usage examples of basic interactions with the "afis" command-line utility including: matching a file against a database, registering a file and then matching it against the database and garbling the image prior to matching.

user.

Most functionality is invoked using the "afis" command-line utility with the desired options. Examples of the simplest interaction possible with the system are shown in Figure 7.3. Included are commands for matching a fingerprint image with the default options, matching a fingerprint image while also registering it and finally garbling an image before matching it against the database.

All matching commands using "afis" produce a newline separated list of the basenames ${ }^{3}$ of fingerprint images which match that of the file specified on the commandline.

The selection of an alternative method for minutia matching can be chosen using the options ("-a" and "-algorithm") exemplified in Figure 7.4. Also shown are the methods of specifying the parameters ("-k", "w", and "-p") of those garbling algorithms that require them.

In addition to selection of the algorithm(s) used for matching, the algorithms used for pre-processing and feature extraction can also be specified. This permits simple customization of much of the process used by the system for matching fingerprints. Examples of the specification of alternative algorithms for pre-processing and feature extraction are shown in Figure 7.5.

\footnotetext{
${ }^{3}$ The "base-name" of a fingerprint in the database refers to the name of the file which was used to register the fingerprint in the database without the file extension.
} 


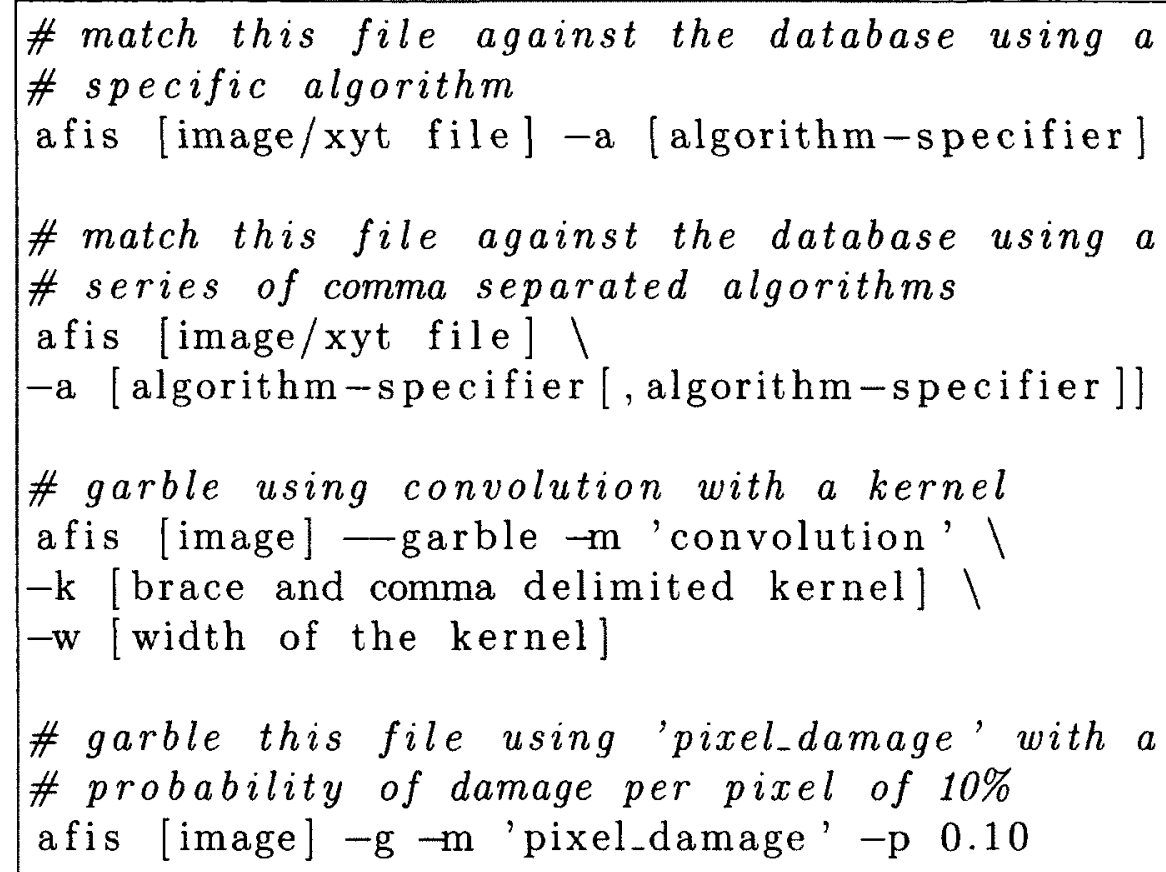

Figure 7.4: Usage examples of several more advanced options of the "afis" utility including: selection of the matching algorithm(s), specifying noise options, specifying options to the garbling algorithms.

\# match this file against the database using a afis [image] -feature-extraction [algorithm-specifier]

Figure 7.5: Usage examples of supplying different algorithms for pre-processing and feature extraction. 


\subsection{Chapter Summary}

Through the course of this chapter we have covered the features of the system, including a detailed description of the fingerprint library and its implementation and the piping mechanism which can be used to build hierarchical fingerprint matching systems. Apart from presenting an example of how the fingerprint library could be used, we also presented a description of how each component of the system relates to the original structure of a typical biometric system. Finally we presented a series of usage examples to illustrate a user's common interactions with the system. 


\section{Chapter 8}

\section{Conclusions}

The aim of the work presented in this thesis was to develop a complete fingerprint recognition system and to implement and test a novel approach to fingerprint recognition. To establish the necessary foundation for addressing this problem, throughout the course of this thesis, we have included a detailed background of the field of fingerprint recognition, an overview of the components which constitute a biometric system, and a review of several fingerprint matching algorithms of both historical and technological significance. With this serving as a background, we developed and evaluated a novel and pioneering NSTRA-based scheme, implemented a prototype of the NSTRA-based algorithm, and realized a rational specification of the overall implemented fingerprint recognition system.

\subsection{Thesis Summary}

In Chapter 2 we described the nature of biometric systems including their characteristics, and the trade-offs between various design decisions. We also discussed why fingerprints are effective biometrics, and the issues related to their distinctiveness. Finally, we presented, in detail, the process used by fingerprint recognition systems for matching fingerprints.

In Chapter 3 we covered the image processing aspects involved in fingerprint recognition. As well, we reviewed algorithms and tools used in typical applications 
of fingerprint recognition.

In Chapter 4 we discussed challenges faced when storing large fingerprint image databases and the common approaches to overcoming them. We also discussed the file formats supported and the tools and libraries used to accomplish the conversion between different formats. Such aspects of file storage and retrieval are essential for establishing a fingerprint recognition system that could be successfully integrated with existing fingerprint libraries.

In Chapter 5 we reviewed several different algorithms of both historical and technological significance, and discussed, in detail, the strategies and algorithms used in each of the matching techniques included in our system, as well as the challenges faced during the implementation phase.

In Chapter 6 we outlined the development of our novel and pioneering fingerprint recognition algorithm based on the NSTRA. We described the reasoning and the benefits of our approach, as well as the implementation details of the algorithm itself. We also tested the algorithm's performance relative to that of the other algorithms specified and implemented in Chapter 5, and provided a measure of the algorithm's performance in a simulated system.

In Chapter 7 we described the overall features of our fingerprint recognition system. We covered the various aspects of its design and implementation, and the rationale behind our design process. We also specified how each component of our system matches with the abstract model of a biometric system presented in Chapter 2.

\subsection{Summary of Contributions}

The first contribution of this thesis is a comprehensive library for fingerprint recognition. It is a flexible, extensible, and high-level fingerprint recognition library permitting the quick assembly of a fingerprint recognition system while hiding the complexity of dealing with fingerprint images themselves. The details of the conversion between appropriate file formats, image pre-processing and minutia-extraction are all, by default, hidden from the developer. The library also provides parallelism and 
the construction of arbitrary hierarchical fingerprint matching algorithms.

Our library used the "fprint" library to interface with fingerprint scanners, and the "cwsq" and "dwsq" programs to convert between the RAW formats provided by fingerprint scanners into those used by other fingerprint recognition tools and packages. Conversion to other supported formats (PNG, JPEG, TIFF) was achieved through the use of the PIL. Image pre-processing was accomplished through both the implemented garbling algorithms and through the "mindtct" program.

Our second contribution is a complete fingerprint recognition system created with our fingerprint recognition library. As with the fingerprint library from which it was constructed, our fingerprint recognition system is scriptable, extensible and capable of parallelism.

Our fingerprint recognition system included a set of minutia matching algorithms comprised of the Hough transform-based algorithm, the K-plet representation-based algorithm and Bozorth's algorithm. The Hough transform-based algorithm was implemented from its specification in [34] and [24], the K-plet representation-based algorithm was ported from a previous implementation reported in [6] to our system, and finally, Bozorth's algorithm was obtained from the NBIS package.

As part of the development of this fingerprint recognition system we have implemented garbling algorithms based on additive Gaussian noise, convolution with arbitrary kernels, and "salt-and-pepper" pixel damage. We have also implemented a mechanism permitting the "piping" of results from one algorithm to another.

Our third contribution was the implementation of a novel and pioneering fingerprint recognition algorithm based on a solution (the NSTRA) to the noisy subsequence tree recognition problem (NSTRP) reported in [5]. We adapted the NSTRA to the fingerprint recognition problem by encoding minutia information into noise tolerant trees.

As part of our adaptation of the NSTRA, we also developed a method of encoding fingerprint minutia information into these noise-tolerant trees. In addition to its use in the NSTRA-based algorithm, we believe that it could prove effective when combined with other matching techniques. 


\subsection{Summary of Conclusions}

The developed fingerprint matching system was complete in that it supported matching of fingerprint templates produced from fingerprint images. It was capable of handling several different image types and was capable of matching fingerprints in a hierarchical manner. While its functionality was complete enough for it to be considered as a stand-alone fingerprint matching system, its only interface was a command-line utility. This is certainly acceptable from the standpoint of a developer or even an "expert" user, but is less desirable from the perspective of a "regular" user. A graphical user interface is currently being developed for the system which, we believe, would alleviate this usability problem.

Although our novel fingerprint matching technique is not yet fully complete as it is missing appropriate noise compensation parameters for a specific application, it does perform better than expected without data pertaining to the noise applied by its environment. Although its false positive rate is high, it is still better than a scheme that randomly discriminates between different fingerprints. The evaluation of the complete algorithm using artificial noise and a simulated environment demonstrated that the algorithm has significant discriminating power and certainly warrants further study. By including noise considerations into the design, we expect improvements to the performance of the algorithm when used in a real fingerprint recognition system. In a closed fingerprint verification system, it could be possible to determine an acceptable range of parameters for the algorithm's noise considerations in order to take advantage of its discriminatory power.

In addition to the developed algorithm, the proposed method of constructing trees from sets of minutiae may useful when combined with other matching techniques. A method similar in spirit to that presented in [6] (which was intended for use with specific graphs) could be adapted to the trees produced by our method. The features of the trees produced by our method render it useful for fingerprint recognition since it eliminates the effects of rotation, and dampens the effect of displacement distortion. 


\subsection{Future Work}

The fingerprint recognition system developed as part of this thesis supports the creation of hierarchical matching techniques. One potential area of study could be the determination of benefits of using hierarchical techniques. This could provide some value or insight into the weaknesses/strengths of different fingerprint matching techniques, and potentially allow the synthesis of more schemes.

There are a number of improvements that could be made to the fingerprint recognition system developed in this thesis. The interface, although complete in its support of the systems features, is rudimentary in the sense that it would only be usable by

a developer or expert user. A graphical user interface (GUI) is in development to alleviate this issue. The GUI will be web-based and will support remote interaction with the system.

As previously stated, the most concerning aspect of the NSTRA-based algorithm's performance is its high FPR. If the cause of the high FPR is a result of discarding some global spatial relationships, a consolidation step in which more evidence is gathered and cross-referenced with previous conclusions, could significantly reduce the FPR. It is also possible that the high FPR is a result of the incomplete state of the algorithm. In this case, more detailed knowledge of the noise inherent in a fingerprint matching system would permit increasing the performance of the algorithm.

The algorithm is, as of yet, complete but not perfected. It is our opinion that the nature of the noise present in fingerprint images must be studied in order to fully take advantage of the NSTRA-based algorithm's discriminatory power. The experiments performed on the NSTRA-based algorithm, which utilized artificial noise, indicated that it possessed a significant level of discriminatory power. It is difficult to determine exactly where its performance lies when compared to the other algorithms discussed in this thesis as the artificial noise experiments were performed in a very different and controlled environment. Nevertheless, we believe that these results warrant further study. The exact nature of the noise may not be necessary, because an approximation would, in our opinion, improve the performance of the algorithm in a complete fingerprint recognition system. 


\section{Bibliography}

[1] A. K. Jain and D. Maltoni, Handbook of Fingerprint Recognition, Springer-Verlag New York, Inc., Secaucus, NJ, USA, 2003.

[2] H. Lin, W. Yifei, and A. K. Jain, "Fingerprint Image Enhancement: Algorithm and Performance Evaluation," IEEE Transactions on Pattern Analysis and Machine Intelligence, vol. 20, no. 8, pp. 777 - 789, Aug 1998.

[3] A. Fareio, A History of Fingerprints, Interpol, Apr 2009.

[4] A. K. Jain, A. Ross, and S. Prabhakar, "An Introduction to Biometric Recognition," IEEE Transactions on Circuits and Systems for Video Technology, vol. 14, no. 1, pp. 4-20, Jan 2004.

[5] B. J. Oommen and R. K. S. Loke, "On the Pattern Recognition of Noisy Subsequence Trees," IEEE Transactions on Pattern Analysis and Machine Intelligence, vol. 23, no. 9, pp. 929 - 946, Aug 2001.

[6] N. K. Ratha, R. M. Bolle, V. D. Pandit, and V. Vaish, "Robust Fingerprint Authentication Using Local Structural Similarity ," in Applications of Computer Vision, 2000, Fifth IEEE Workshop on., 2000, pp. $29-34$.

[7] J. L. Wayman, "Technical Testing and Evaluation of Biometric Identification Devices," in Biometrics, A. K. Jain, R. Bolle, and S. Pankanti, Eds., pp. 345 368. Springer US, 2002. 
[8] Federal Bureau of Investigation, "The Integrated Automated Fingerprint Identification System," Online http://www.fbi.gov/about-us/cjis/ fingerprints_biometrics/iafis/iafis, Apr 2011.

[9] B. Burger, D. Fuchs, E. Sprecher, and P. Itin, "The Immigration Delay Disease: Adermatoglyphia-Inherited Absence of Epidermal Ridges," Journal of the American Academy of Dermatology, vol. 64, no. 5, pp. 974 - 980, May 2011.

[10] S. Pankanti, S. Prabhakar, and A. K. Jain, "On the Individuality of Fingerprints," IEEE Transactions on Pattern Analysis and Machine Intelligence, vol. 24, pp. 1010 - 1025, Aug 2002.

[11] C. Doctorow, "Walt Disney World Fingerprints Visitors," Online http:// boingboing.net/2006/09/01/walt-disney-world-fi.html, Apr 2011.

[12] L. Spadanuta, K. Harmel, and Associated Press, "Disney World Scans Fingerprint Details of Park Visitors," Online http://www.boston.com/news/ nation/articles/2006/09/03/disney_world_scans_fingerprint_details_ of_park_visitors/, Apr 2011.

[13] K. Harmel, "Walt Disney World: The Government's Tomorrowland?," Online http://newsinitiative.org/story/2006/09/01/walt_disney_world_the_ governments, Apr 2011.

[14] Mythbusters, "Crimes and Myth-Demeanors 2," TV, Beyond Television Productions, Aug 2006, Season 04, Episode 04, A well reputed fingerprint security system is put to the test.

[15] S. A. Cole, "Is Fingerprint Identification Valid? Rhetorics of Reliability in Fingerprint Proponents Discourse," Law \& Policy, vol. 28, no. 1, pp. 109 - 135, 2006.

[16] A. K. Jain, S. Prabhakar, and S. Pankanti, "On the Similarity of Identical Twin Fingerprints," Pattern Recognition, vol. 35, no. 11, pp. 2653-2663, Nov 2002. 
[17] F. Galton, "Personal Identification and Description," The Journal of the Anthropological Institute of Great Britain and Ireland, vol. 18, pp. 177 - 191, 1889.

[18] W. J. Herschel, "Skin Furrows of the Hand," Nature, vol. 23, no. 578, pp. 76, Nov 1880.

[19] N. Grew, "The Description and Use of the Pores in the Skin of the Hands and Feet, by the Learned and Ingenious Nehemiah Grew, M. D. Fellow of the College of Physicians and of the Royal Society," Philosophical Transactions, vol. 14, no. 155-166, pp. 566 - 567, Jan 1684.

[20] Federal Bureau of Investigation, "Electronic Biometric Transmission Specification," Criminal Justice Information Services, May 2010.

[21] T. Reed and R. Meier, "Taking Dermatoglyphic Prints. A Self-Instruction Manual.," Amer Dermatologlyphic Assoc Newsletter Suppl, pp. 1-45, 1990.

[22] H. C. Lee and R. E. Gaensslen, "Advances in Fingerprint Technology," Elsevier Science, p. 290, 2001.

[23] R. D. Bahuguna and T. Corboline, "Prism Fingerprint Sensor That Uses a Holographic Optical Element," Applied Optics, vol. 35, no. 26, pp. $5242-5245$, Sep 1996.

[24] N. K. Ratha, K. Karu, C. Shaoyun and A. K. Jain, "A Real-Time Matching System for Large Fingerprint Databases," IEEE Transactions on Pattern Analysis and Machine Intelligence, vol. 18, no. 8, pp. 799 - 813, Aug 1996.

[25] S. Chikkerur, A. Cartwright, and V. Govindaraju, "K-Plet and Coupled BFS: A Graph Based Fingerprint Representation and Matching Algorithm," in Advances in Biometrics, D. Zhang and A. K. Jain, Eds., vol. 3832, pp. 309 - 315. Springer Berlin / Heidelberg, 2005.

[26] N. K. Ratha, S. Chen, and A. K. Jain, "Adaptive Flow Orientation-Based Feature Extraction in Fingerprint Images," Pattern Recognition, vol. 28, no. 11, pp. 1657 - 1672, Nov 1995. 
[27] R. C. Gonzalez and R. E. Woods, Digital Image Processing, Prentice Hall, Upper Saddle River, NJ, USA, 2nd edition, 2002.

[28] B. M. Mehtre, N. N. Murthy, S. Kapoor, and B. Chatterjee, "Segmentation of Fingerprint Images Using the Directional Image," Pattern Recognition, vol. 20, no. 4 , pp. $429-435,1987$.

[29] A. K. Jain, S. Lin, H. Pankanti, and R. Bolle, "An Identity-Authentication System Using Fingerprints," Proceedings of the IEEE, vol. 85, no. 9, pp. 1365 1388, Sep 1997.

[30] A. K. Jain and H. Lin, "On-Line Fingerprint Verification," in Pattern Recognition, 1996., Proceedings of the 13th International Conference on, Aug 1996, vol. 3 , pp. $596-600$.

[31] A. K. Jain, Y. Chen, and M. Demirkus, "Pores and Ridges: High-Resolution Fingerprint Matching Using Level 3 Features," IEEE Transactions on Pattern Analysis and Machine Intelligence, vol. 29, no. 1, pp. 15 - 27, Jan 2007.

[32] C. M. Brislawn, J. N. Bradley, R. J. Onyshczak, and T. Hopper, "FBI Compression Standard for Digitized Fingerprint Images," Spie, vol. 2847, no. 344, pp. $344-355$, Nov 1996.

[33] T. Hopper, C. Brislawn, and J. Bradley, "WSQ Gray-Scale Fingerprint Image Compression Specification," IAFIS-IC-0110-V2, vol. 2, Feb 1993.

[34] D. H. Ballard and C. M. Brown, Computer Vision, Prentice-Hall, Englewood Cliffs, NJ, 1982.

[35] A. K. Hrechak and J. A. McHugh, "Automated Fingerprint Recognition Using Structural Matching," Pattern Recognition, vol. 23, no. 8, pp. 893-904, 1990.

[36] Z. Chen and C. H. Kuo, "A Topology-Based Matching Algorithm for Fingerprint Authentication," in 25th Annual 1991 IEEE International Carnahan Conference on Security Technology, 1991. Proceedings., Oct 1991, pp. $84-87$. 
[37] K. C. Fan, C. W. Liu, and Y. K. Wang, "A Randomized Approach with Geometric Constraints to Fingerprint Verification," Pattern Recognition, vol. 33, no. 11, pp. $1793-1803,2000$.

[38] J. T. Tou and R. C. Gonzalez, "Pattern Recognition Principles," Image Rochester $N Y$, vol. 7, pp. 377, 1974.

[39] C. I. Watson, M. D. Garris, E. Tabassi, C. L. Wilson, R. M. McCabe, S. Janet, and K. Ko, User's Guide to Export Controlled Distribution of Nist Biometric Image Software, National Institute of Standards and Technology, Gaithersburg, MD, USA, Sep 2004.

[40] The National Institute of Standards and Technology, "Nist FAQ," Online http: //www.nist.gov/itl/iad/ig/slapseg04_faq.cfm, Jan 2011. 